\title{
Behavioral and imaging markers of multiple sclerosis
}

\author{
Ph.D. Thesis
}

Bálint Kincses M.D.

Clinical and Experimental Neuroscience Program, Doctoral School of Clinical Medicine Department of Neurology Faculty of Medicine Albert Szent-Györgyi Clinical Center University of Szeged

Supervisor: Zsigmond Tamás Kincses M.D., Ph.D., D.Sc

Department of Radiology, Faculty of Medicine Albert Szent-Györgyi Clinical Center University of Szeged 


\section{Original Publications directly related to the thesis:}

I. Kincses, B.; Herak, B.J.; Szabo, N.; Bozsik, B.; Farago, P.; Kiraly, A.; Vereb, D.; Toth, E.; Kocsis, K.; Bencsik, K.; Vecsei, L.; Kincses, Z.T. Gray Matter Atrophy to Explain Subclinical Oculomotor Deficit in Multiple Sclerosis, Frontiers in neurology (2019) DOI: 10.3389/fneur.2019.00589 IF: $\mathbf{2 . 6 3 5}$

II. Kincses, B.; Spisak, T.; Farago, P.; Kiraly, A; Szabo, N.; Vereb, D; Kocsis, K.; Bozsik, B.; Toth, E.; Vecsei, L.; Kincses, Z.T. Brain MRI diffusion encoding direction number affects Tract-Based Spatial Statistics results in multiple sclerosis, Journal of Neuroimaging (2020) DOI: 10.1111/jon.12705, IF: 2.08

\section{Cumulative impact factor of the publications directly related to the thesis: $\mathbf{4 . 7 1 5}$}

\section{Original Publications not directly related to the thesis:}

I. Spisak, T.; Kincses, B.; Schlitt, F.; Zunhammer, M.; Schmidt-Wilcke, T.; Kincses, Z.T.; Bingel, U. Pain-free resting-state functional brain connectivity predicts individual pain sensitivity, Nature Communications (2020) DOI: 10.1038/s41467019-13785-z, IF: 11.878

II. Vereb, D.; Szabo, N.; Tuka, B.; Tajti, J.; Kiraly, A.; Farago, P.; Kocsis, K.; Toth, E.; Bozsik, B.; Kincses, B.; Vecsei, L.; Kincses, Z.T. Temporal instability of salience network activity in migraine with aura, Pain (2019) DOI: 10.1097/j.pain.0000000000001770, IF: 6.029

III. Farago, P.; Toth, E.; Kocsis, K.; Kincses, B.; Vereb, D.; Kiraly, A.; Bozsik, B.; Tajti, J.; Pardutz, A.; Szok, D.; Vecsei, L., Szabo, N.; Kincses, Z.T. Altered Resting State Functional Activity and Microstructure of the White Matter in Migraine With Aura, Frontiers in neurology (2019), DOI: 10.3389/fneur.2019.01039, IF: 2.635

IV. Kincses, Z.T.; Vereb, D.; Farago, P.; Toth, E.; Kocsis, K.; Kincses, B.; Kiraly, A.; Bozsik, B.; Pardutz, A.; Szok, D.; Tajti, J.; Vecsei, L.; Tuka, B.; Szabo, N. Are Migraine With and Without Aura Really Different Entities? Frontiers in neurology (2019) DOI: 10.3389/fneur.2019.00982, IF: 2.635

V. Toth, E.; Farago, P.; Kiraly, A.; Szabo, N.; Vereb, D.; Kocsis, K.; Kincses, B.; Sandi, D.; Bencsik, K.; Vecsei, L.; Kincses, Z.T. The Contribution of Various MRI Parameters to Clinical and Cognitive Disability in Multiple Sclerosis, Frontiers in neurology (2019), DOI: 10.3389/fneur.2018.01172, IF: 2.635

VI. Szabo, N.; Farago, P.; Kiraly, A.; Vereb, D.; Csete, G.; Toth, E.; Kocsis, K.; Kincses, B.; Tuka, B.; Pardutz, A.; Szok, D.; Tajti, J.; Vecsei, L.; Kincses, Z.T. Evidence for Plastic Processes in Migraine with Aura: A Diffusion Weighted MRI Study, Frontiers in neuroanatomy (2018), DOI: 10.3389/fnana.2017.00138, IF: 2.923

VII. Vereb, D.; Szabo, N.; Tuka, B.; Tajti, J.; Kiraly, A.; Farago, P.; Kocsis, K.; Toth, E.; Kincses, B.; Bagoly, T.; , Helyes, Z.; Vecsei, L.; Kincses, Z.T. Correlation of neurochemical and imaging markers in migraine. PACAP38 and DTI measures, Neurology (2018), DOI: 10.1212/WNL.0000000000006201, IF: 8.689

VIII. Kincses, Z.T.; Farago, P.; Szabo, N.; Vereb, D.; Kiraly, A.; Toth, E.; Kocsis, K.; Kincses, B.; Vecsei, L. Struktúrális és funkcionális MRI vizsgálatok fejfájás 
betegségekben - irodalmi áttekintés és saját kutatási eredmények. Cephalalgia hungarica (2016)

IX. Szabo, N.; Tuka, B.; Vereb, D.; Kiraly, A.; Farago, P.; Toth, E.; Kocsis, K.; Kincses, B.; Vecsei, L.; Kincses, Z.T. Molekuláris és képalkotó biomarkerek összefüggése fejfájás betegségekben - irodalmi áttekintés és saját kutatási eredmények. Cephalalgia hungarica (2016)

Cumulative impact factor of the publications not directly related to the thesis: $\mathbf{3 7 . 4 2 4}$

Total impact factor: $\mathbf{4 2 . 1 3 9}$ 


\section{Table of contents}

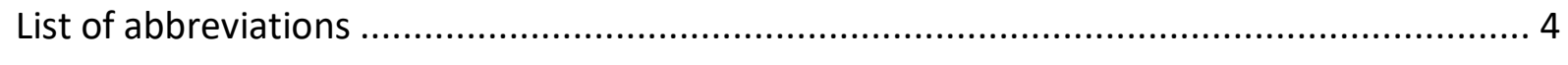

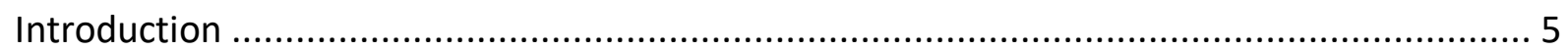

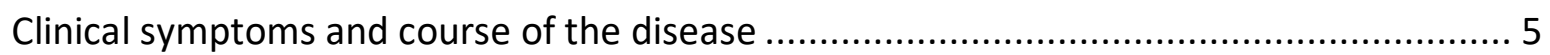

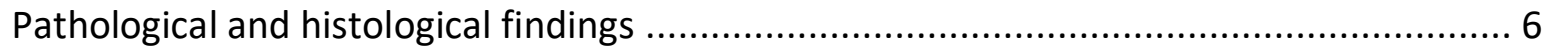

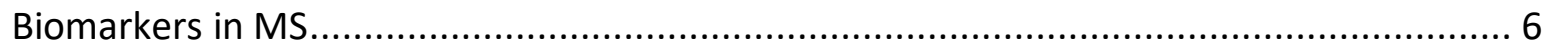

MRI characteristics and relation to clinical condition .................................................... 7

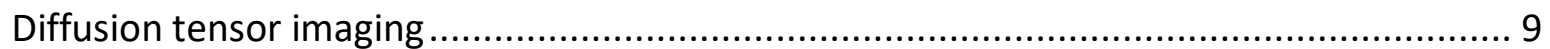

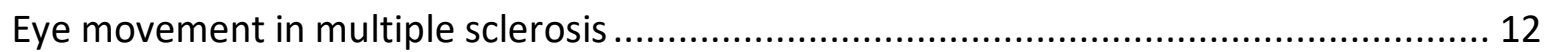

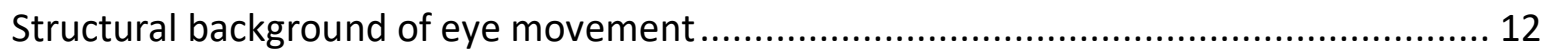

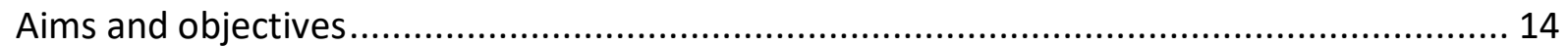

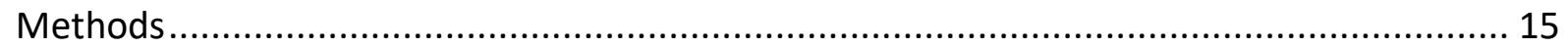

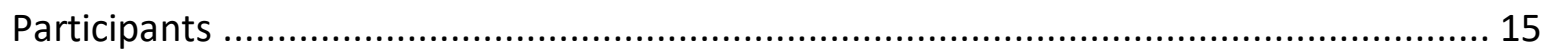

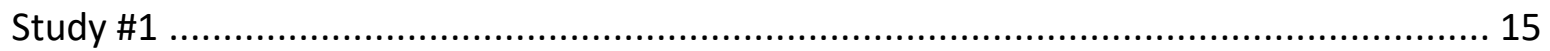

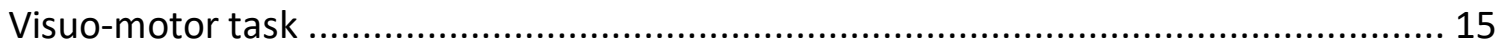

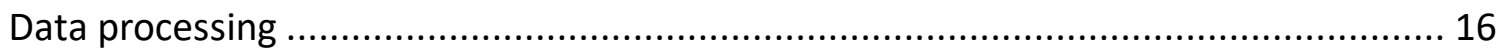

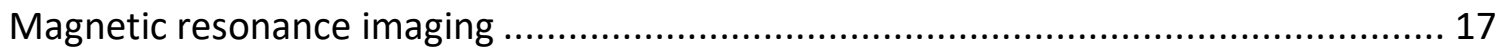

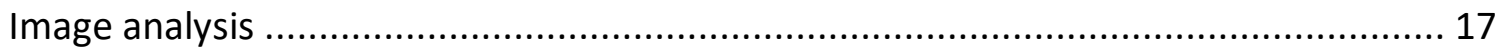

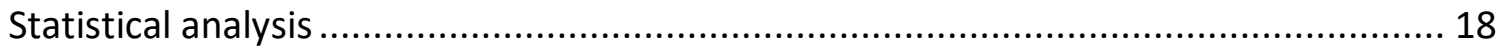

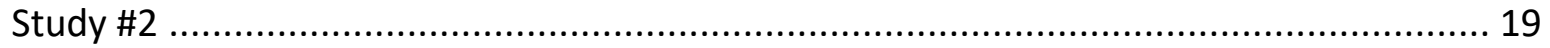

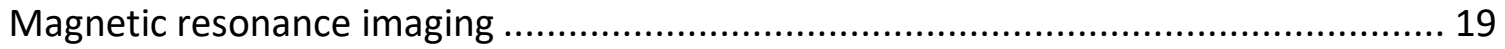

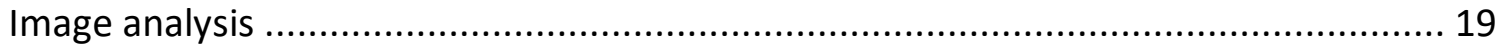

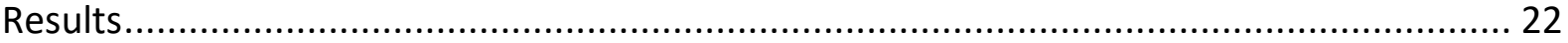

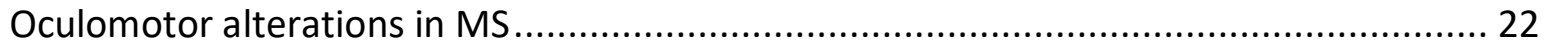

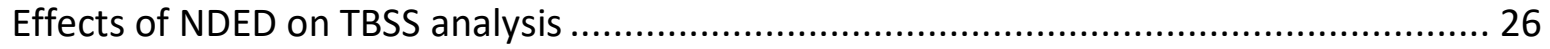

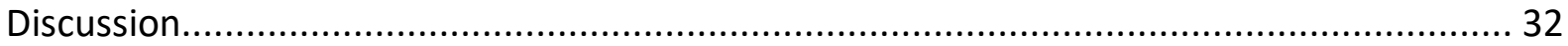

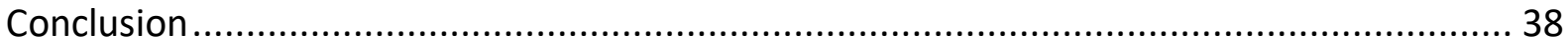

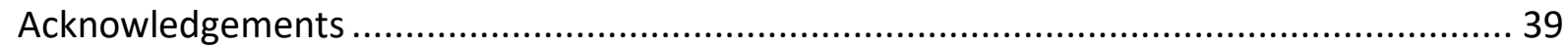

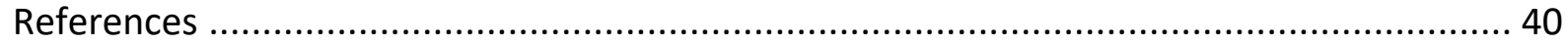




\section{List of abbreviations}

CIS

CNS

$\mathrm{D}$

DIR

DOF

EDSS

FEF

FOV

FSPGR

Gd

GLM

$\mathrm{HC}$

INO

IR

NDED

NEX

MRI

MS

PPMS

RRMS

RIS

ROI

SE

SNR

sl

SPMS

T2W

TE

TI

TR

VBM
Clinically isolated syndrome

Central nervous system

Diffusion coefficient

Double inversion recovery

Degrees of freedom

Expanded disability status scale

Frontal eye field

Field of view

Fast spoiled gradient echo

Gadolinium

General linear model

Healthy controls

Internuclear ophtalmoparesis

Inversion recovery

Number of diffusion

encoding directions

Number of excitation

Magnetic resonance imaging

Multiple sclerosis

Primary progressive MS

Relapsing remitting MS

Radiologically isolated symptoms

Region of interest

Spin echo

Signal-to-noise ratio

Slice thickness

Secondary progressive MS

T2 weighted

Echo time

Inversion time

Repetition time

Voxel based morphometry 


\section{Introduction}

\section{Clinical symptoms and course of the disease}

Multiple sclerosis is a devastating disease which mostly affects young adults and it is more common in females (the male female ratio is 1:3). Genetic and environmental factors are jointly responsible for developing the disease ${ }^{1}$. This complex interplay leads to an autoimmune inflammation of the nervous system. The focal and diffuse inflammation and consequential changes cause the various clinical symptoms. While the white matter lesions are characteristic for the disease, diffuse microstructural and neurodegenerative alteration occur as well. A variety of symptoms presents such as optic neuritis, brainstem syndromes or sensory and motor disturbances. The expanded disability status scale (EDSS) is an established tool to evaluate clinical severity ${ }^{2}$. It grades clinical condition in eight functional systems such as pyramidal, cerebellar, brainstem and sensory systems. EDSS aids the monitoring of the disease, test therapeutic efficacy and improves clinical research. Therefore, in itself fulfils many of the desired features of a biomarker (see Biomarkers in MS). It is easy to administer, widely used clinically and requires no additional equipment. Besides the many advantages, there are few limitations of the scale. EDSS is highly dependent on mobility and it is nonlinear in terms of time spent at certain stages of the scale. Moreover, the scale is insensitive to small changes. Certain functions are underrepresented such as cognition and oculomotor deficits. Nystagmus is included in the scale, but other frequently found alterations in MS are missing such as internuclear ophtalmoparesis (INO), strabismus, impaired smooth pursuit and disorders of saccades $^{3}$ (see Eye movement in MS section).

The most frequent form of MS is the relapsing remitting (RRMS) form. In a relapse, symptoms develop from hours to days. In the natural course, it is followed by a plateau phase for several weeks and gradually ameliorate. Usually, the full recovery is not reached, some partial damage is still detectable. In a preliminary state of the disease, namely in the clinically isolated syndrome (CIS), the diagnostic criteria are not fulfilled. When the disease is only suspected, MS presents as CIS. After onset of RRMS, secondary progressive (SP) form commonly develops within 15 years. The continuous progression of symptoms become more dominant. In 5-15\% of cases, the disease presents with a more severe, the primary progressive (PP) form. In addition, the widespread use of MRI has increased incidental brain abnormalities. 
The phenomenon radiologically isolated syndrome (RIS) for asymptomatic subjects with MRI abnormalities highly suggestive for MS is introduced ${ }^{4}$.

The natural course of the disease could be modified with several agents. The therapeutic intervention could be separated as therapy of acute relapses, disease modifying therapies and symptomatic treatments. In the therapy of acute phase, the first line treatment is intravenous methylprednisolone ${ }^{5}$. In refractory cases, plasmapheresis or intravenous immunoglobulin therapy could be administered. To prevent relapses and decrease disease activity numerous disease modifying therapy is available. Immunosuppressants (fingolimod, natalizumab, ocrelizumab), immunomodulatory (interferon-beta, glatiramer acetate) and immune reconstitution agents (alemtuzumab, cladribine) are all used in MS treatment ${ }^{1}$. The efficacy, side effect profile and way of administration of the agents are different.

\section{Pathological and histological findings}

The very first described brain pathology in MS were the macroscopic brain lesions ${ }^{6}$. The microscopic investigation of these lesions revealed that inflammation and demyelination occur $^{7}$. Most of the infiltrate consist of T cells but B lymphocytes and plasma cells are also found. The immune cells cause the damage of oligodendrocytes, which leads to demyelination. The axons are typically involved in the later stages of the disease but there are evidences that neurodegeneration also appears at early stages of $\mathrm{MS}^{8}$. The perivenular inflammation is followed by remyelinating processes. The pathological alterations could not distinguish MS subtypes, instead it creates a continuum. To conclude both inflammation and neurodegeneration secondary to inflammation and per se occur as well.

\section{Biomarkers in $M S$}

A biomarker is a trait, which is measured objectively and assessed as an indicator of physiological and pathological processes or the response to a therapeutic intervention ${ }^{9}$. Biomarkers in general are applicable for the diagnosis of a disease, monitoring treatment effectiveness or disease progression. Several important features of a good biomarker are established. A biomarker should be measured robustly and precisely and with high sensitivity and specificity. It correlates disease pathology and is cost effective ${ }^{10}$. Two phases of biomarker development has to be distinguished. The discovery phase refers to the search for a biomarker with relatively small sample size and robust statistical methods. In the validation phase, the previously found putative biomarker is verified in large sample size. As MRI is used in the 
diagnostic evaluation, monitoring and differential diagnosis of MS, a great number of research investigated the potential role of MRI markers in MS biomarker research. For example, a new lesion in fluid attenuated inversion recovery (FLAIR)/T2 weighted image in CIS means that the disease converts to RRMS. Its relation with the clinical status is ambiguous. In contrary, gray matter abnormality is a good marker to clinical disability ${ }^{11}$. Brain atrophy could differentiate MS brain from healthy ${ }^{12}$.

\section{MRI characteristics of MS and relation to clinical condition}

With the advent of magnetic resonance imaging, many MRI measures have been associated with previously described histopathological findings. In addition, MS biomarker research tested these measures how they reflect clinical condition, predict impairment and therapeutic response.

Gadolinium $(\mathrm{Gd})$, a widely used MRI contrast agent, shortens $\mathrm{T}_{1}$ relaxation time of adjacent protons. Therefore, in sites of Gd accumulation hyperintense area could be observed in $\mathrm{T}_{1}$ weighted images. Gd enhancing lesions are found in areas where blood-brain barrier breakdown and inflammation are described histopathologically in MS patients ${ }^{13}$. The enhanced areas are hyperintense in $T_{2}$ weighted images and hypointense in unenhanced $T_{1}$ weighted images. An enhancing lesion could be observed 3 weeks in average and disappear thereafter ${ }^{14}$. Enhancement usually precedes a newly forming lesion ${ }^{13,14}$. The presence of an enhancing lesion is a predictor of relapse occurrence ${ }^{15}$. Moreover, it is a weak predictor for long term, cumulative disability and impairment ${ }^{15,16}$. These observations reflect that ongoing inflammation in MRI involved in the occurrence of a relapse, but other pathological mechanisms such as atrophy play a role in long-term disability.

The FLAIR and $\mathrm{T}_{2}$-weighted sequences are used to identify the hyperintense lesions. FLAIR sequence suppress free water (e.g. cerebrospinal fluid), therefore periventricular lesion are delineated more precisely. Another advantage of FLAIR over $\mathrm{T}_{2} \mathrm{~W}$ images is that it visualize cortical and juxtacortical lesions in a greater amount ${ }^{17}$. The formation of lesions anywhere in the central nervous system (CNS) reflects the disseminated nature of the disease. The typical lesions in MS are rounded/oval in shape and extend at least $3 \mathrm{~mm}$ in their main axis ${ }^{18}$. Their distribution usually is asymmetric at early stage but both hemispheres are affected. Lesions occur anywhere in the CNS, but certain regions are more prone to lesion formation such as the periventricular region, juxtacortical white matter, corpus callosum, infratentorial region and the 
spinal cord ${ }^{18}$. The optic nerves are also frequently affected. The hyperintense lesions in general only modestly reflect the clinical disability in $\mathrm{MS}^{19}$, therefore, other measures are necessary to attain a more comprehensive description of clinical impairment.

Some of the hyperintense lesions in FLAIR/ $\mathrm{T}_{2} \mathrm{~W}$ images are hypointense in the $\mathrm{T}_{1}$ weighted images, which refers to the widely used "black hole" phenomenon. The investigation of black hole dynamics revealed that some of them only temporary and turn isointense later ${ }^{20}$. They indicate severe demyelinization, axonal loss and matrix destruction ${ }^{16,19,21}$. Larger Gd enhancing lesions more frequently appear as temporary hypointensities ${ }^{22}$, which reflects that more severe inflammation leads to tissue destruction. The clinical condition is in modest-tostrong association with the black hole status, and it is better than the $\mathrm{T}_{2}$ hyperintense lesions ${ }^{19}$. In addition, it is hypothesized that black hole burden is a good marker for progressive tissue damage and clinical progression ${ }^{23}$.

Besides the lesions, other structural abnormalities are detected. Brain and spinal cord atrophy turned out to be a good biomarker of MS. The tissue loss may reflect a global measure of pathology and the reserve capacity of the CNS as well. It predicts long-term disability ${ }^{24}$ and related to neuropsychological impairment ${ }^{25}$. The gray matter atrophy alone is associated with clinical disability ${ }^{19,26}$.

The white matter, in which no lesion is detected, the so-called normal appearing white matter is affected as well. Microstructural alterations could be observed with special MRI sequences such as diffusion tensor imaging ${ }^{27}$ and magnetic transfer imaging ${ }^{28}$. The underlying inflammation and demyelination are responsible for the observed changes.

Several measurable MRI parameters are identified which are deteriorated in the disease. These alterations represent the inflammatory or the neurodegenerative nature of the disease, or both. For example, atrophy or black holes are associated with neurodegeneration and tissue loss. It is not clear that neurodegeneration or inflammation or both together cause clinical disability. In remission, in which disease activity is decreased, clinical symptoms persist. The white matter lesions, which usually consider to reflect the inflammatory part of MS, are not accountable for the clinical condition solely. The neurodegenerative factors are also partly account for the symptoms. Hence, the combination of imaging markers could predict the clinical status. Moreover, to elucidate which mechanism in what extent is in the background of certain clinical symptoms such as oculomotor deficit may aid the selection of future neuroprotective or remyelinating therapies ${ }^{29}$. 


\section{Diffusion tensor imaging}

Diffusion imaging is based on the molecular motion of protons. Diffusion is the nonbulk motion of molecules which occur at any temperature and should be distinguished from bulk motion such as convection. Robert Brown demonstrated this phenomenon in the 19th century ${ }^{30}$. Pollen suspended in liquid medium exhibit irregular motion which could be observed under microscope. The first mathematical description of diffusion has been dated back the mid$19^{\text {th }}$ century. In classical physics, Fick's law describes that the diffusion flux is proportional with the concentration gradient ${ }^{31}$. They are related by the factor $\mathrm{D}$, which is called the diffusion coefficient. The Gaussian distribution of motion is hypothesized in the probabilistic model of diffusion which was first described by Einstein ${ }^{32}$. Within this model, it is showed that the displacement of a particle over a period of time is proportional with the diffusion coefficient. It was derived that the diffusion coefficient is proportional with the temperature, the particle size and the viscosity of medium. The simplest form of diffusion is isotropic which means that particles move in any directions with equal probability. In contrary, in anisotropic medium, there are preferred directions of diffusion. In biological tissues, obstacles such as membranes restrict diffusion of water in directions perpendicular to them and diffusion is preferred in the parallel direction. Highly organized structures such as nerve fiber tracts could be found in the human CNS. The water molecules easily move in the parallel direction, because there are no obstacle in that direction. However, perpendicular to the axons, the lipid membrane restricts the diffusion of water molecules. Measurement of water protons' diffusion in these regions could serve information about the impairment of underlying structures.

In modern diffusion weighted imaging the used MRI sequence is based on the pulsed gradient spin echo technique ${ }^{33}$. There is a pair of diffusion sensitizing gradients which affect stationary and diffusing water protons differently. The stationary protons accumulate phase by the first gradient and reversed equally by the second one as the same amount of gradient is applied. However, diffusing protons change their location between the two gradients and falling out-of-phase. This eventually leads to a decrease in signal intensity that is areas with higher diffusion have lower intensity and restricted areas have higher intensity. Therefore, the intensity of images reflects the amount of diffusion. After the diffusion weighting a fast imaging module is applied, usually an echo planar imaging sequence. Moreover, a fat suppression techniques is used to decrease chemical shift artefacts. The Stejskal-Tanner formula ${ }^{33}$ (Equation 1) describes the signal change when a diffusion gradient is applied. 


$$
S=S_{0} e^{-b D}
$$

Equation 1

In Equation 1, S means the recorded signal intensity after the application of a diffusion gradient, $\mathrm{S}_{0}$ stands for the original signal intensity, $\mathrm{D}$ is the diffusion coefficient and $\mathrm{b}$ is a factor which determines the degree of diffusion weighting. This is set by the MRI operator and is associated with the used diffusion gradient shape. In clinical MRI scans it is usually between 0 and $3000 \mathrm{~s} / \mathrm{mm}^{2}$ depending on the field strength, imaged region and predicted pathology. A rule of thumb that the $b^{*} \mathrm{D} \sim 1$, which means a $b$ value around $1000 \mathrm{~s} / \mathrm{mm}^{2}$ in the brain in clinical scanners. The signal intensities in the equation are measured, the $b$ value is set by the operator, and therefore, the diffusion coefficient could be easily calculated.

In isotropic medium, diffusion is equal in any directions thus it is characterized with a scalar. However, as previously mentioned, biological tissues are frequently anisotropic media. It means that diffusion of water protons are different in certain directions. To rephrase it, the amount of measured diffusion depends on the investigated direction. In clinical practice at least three directions, for instance the main axes of the laboratory frame $(\mathrm{x}, \mathrm{y}, \mathrm{z})$, are used. For the description of direction dependent diffusion coefficient in anisotropic medium a tensor model is introduced ${ }^{34}$. The diffusion tensor is a 3 by 3 symmetrical positive matrix. The diagonal elements are the diffusion coefficients in the main laboratory axes $(\mathrm{x}, \mathrm{y}, \mathrm{z})$ and the off-diagonal elements represent the correlation of diffusion between the main axes. The opposite (e.g. $x-y$ and $\mathrm{y}-\mathrm{x}$ ) directions are equal in terms of diffusion. The geometrical representation of a tensor is an ellipsoid, which shape and surface represent the direction and amount of diffusion. One needs at least six measurements with diffusion weighting and an extra image usually without diffusion weighting to characterize this diffusion tensor. The six non-collinear diffusion directions is sufficient, however, more directions used in practice to increase signal-to-noise ratio (SNR). From the tensor model, several parameters are derived to characterize white matter changes qualitatively. Fractional anisotropy (FA) (Equation 2) describes the diffusion asymmetry within a voxel. In the geometrical representation, FA means how the ellipsoid is elongated in one direction.

$$
F A=\sqrt{\frac{3}{2}} \sqrt{\frac{\left(\lambda_{1}-\lambda_{\text {mean }}\right)^{2}+\left(\lambda_{2}-\lambda_{\text {mean }}\right)^{2}+\left(\lambda_{3}-\lambda_{\text {mean }}\right)^{2}}{\lambda_{1}^{2}+\lambda_{2}^{2}+\lambda_{3}^{2}}}
$$


Mean diffusivity (MD) (Equation 3) and the main eigenvectors $(\lambda 1, \lambda 2, \lambda 3)$ represent the average diffusivity within the voxel and the diffusion along the main diffusion directions.

$$
M D=\frac{\lambda_{1}+\lambda_{2}+\lambda_{3}}{3}
$$

Equation 3

Diffusion tensor imaging (DTI) is a widely used technique to analyze tissue microstructure in vivo. While the spatial resolution of the measurement is in the range of a few millimeters, by choosing the acquisition parameters appropriately one can gather information about the cellular and subcellular structures hindering the diffusion of water. Several studies demonstrated microstructural alterations in various disease ${ }^{35-37}$. As previously mentioned, the correct estimation of the diffusion tensor requires at least 6 non-collinear diffusion directions and an extra usually non-diffusion weighted image. Since diffusion weighted measurement is typically characterized by relatively small SNR, increasing the number of diffusion encoding directions (NDED) is necessary. To estimate tensor orientation and anisotropy independent of SNR one should use a uniform distribution of the encoding directions ${ }^{38}$. Moreover, the optimal NDED is critical for SNR and scan time. More directions increase SNR $^{39}$ but prolong the measurement. The scanning time is especially crucial in a clinical setting, partly because participants may not hold still for longer scan times, which could affect image quality. Several theoretical and practical studies demonstrate the NDED-dependency of the obtained diffusion tensor values and propose an optimum NDED which, depending on the optimization metric, ranges between 6 and $62^{39-46}$.

Although there is a considerable body of research on this topic, there are still outstanding issues to be investigated. Most of these studies evaluated data from healthy controls or used simulated data ${ }^{39-46}$. In vivo studies include the physiological effects (movements, cardiorespiratory effects etc.) and patient related special features (e.g. different in-scanner motion). In addition, special analytical methods could be sensitive to the bias of the tensor value. The widely used tract based spatial statistics (TBSS) is an easy to use and robust method to analyze DTI images ${ }^{47}$. The algorithm is a semi-automatic way to analyze group differences of DTI parameters within the main white matter tracts or test if there is any correlation between these parameters and clinical or behavioral data. In this way, the structural integration or disintegration of white matter is under the scope. The algorithm uses the FA maps of the participants and non-linearly register them. A mean FA map is produced in which the main 
tracts have the highest FA values. After that, all participants individual images are registered to this mean image in a way that the main tracts are aligned together. Therefore, the used statistics is mostly limited on the white matter tracts. Since the publication of the original study, an enormous number of studies used this approach (1737 article cited it according to PubMed in 07/2019). As the method concentrates on the center of the tracks, where the white matter is most uniformly organized, NDED might have specific implications for this analysis technique. However, no previous study investigated the effect of NDED on the TBSS analysis according to our knowledge.

\section{Eye movement in multiple sclerosis}

Oculomotor deficit is common in MS among the many other symptoms and reported to occur in $57-70 \%$ of all patients ${ }^{48,49}$. However, eye movement deficits are underrepresented in EDSS or the multiple sclerosis functional composite. Its significance lies in the observation that the presence of eye movement abnormality is associated with greater disability and greater disability progression ${ }^{50}$. Therefore, disability from eye movement abnormalities is an additional factor in the total disability. Bedside oculomotor examination by an experienced specialist could reveal major oculomotor deficits such as disorder of saccade, disruption of steady fixation, different form of nystagmus. However, subtle alterations might remain undetected. Eye tracker devices are suitable for objective and quantitative measurements of eye movements and are more sensitive to detecting subclinical abnormalities ${ }^{48}$.

\section{Structural background of eye movement}

The aim of eye movements is to keep the object of interest on the fovea. These voluntary and reflexive movements are the rapid jerky saccades, the smooth pursuit and vergence movements. These intricate ocular movements are accomplished by six extraocular muscles, the movement of which is coordinated by a complex network of cortical and subcortical neuronal elements. The main purpose of the rapid voluntary conjugate eye movements known as saccades, is to bring the new object of interest onto the foveae. The cranial nerve nuclei of the oculomotor muscles could be found in the brainstem. In addition, other elements of the premotor circuits of saccades are in the brainstem such as the paramedian pontine reticular formation, nucleus of the medial longitudinal fasciculus and nucleus raphe interpositius. All of these regions receive afferents from the superior colliculus. Moreover, the behaviorally important stimuli are processed in various cortical networks and together with fronto-parietal 
attention networks have crucial role in guiding eye movements during saccades ${ }^{51}$. Eventually the cortical signals for voluntary eye movements are generated in the frontal eye field (FEF) in close interaction with other centers such as supplementary and pre-supplementary eye fields ${ }^{52}$, the dorsolateral prefrontal cortex and parietal cortex. From the cortical centers the information is conveyed via the superior colliculus to the nuclei of the oculomotor nerves directly and indirectly as well ${ }^{53-55}$. Over the course of information flow various subcortical, brainstem and cerebellar centers are modulating the process. Damage to certain parts of this network causes clinically abnormal eye movement ${ }^{3,56}$ some of those easily detectable by bedside examination ${ }^{57}$. However, the structural background of subtle eye movement deficits in MS is not wellunderstood. Damage of the perceptual systems, the cognitive networks such as attention and the eye movement centers cause various alterations of eye movements. The widespread extent of the oculomotor system and close relation with other systems makes it suitable to investigate its alterations, and test its role as a potential biomarker in MS. 


\section{Aims and objectives}

The aims of our studies were to investigate oculomotor deficit in MS patients. The extended network which is responsible for the precise guidance of gaze could be impaired in the early stage of the disease. In addition, the underlying pathology is not well understood. Therefore, we tested MS patients in an in-house built prosaccade and antisaccade task. The higher order pathological background of such abnormal eye movements was investigated by correlating the behavioral measures with MRI parameters such as lesion location and gray matter atrophy.

In order to extend our study to identify the white matter tracts responsible for the altered eye movements, as a first step, we conducted another study to optimize our DTI sequence. The available DTI sequences are frequently time consuming and optimal parameters are necessary to reduce scan time but detect biologically important differences. While NDED is directly proportional to scan time, we decreased it and tested if it changed the applied statistical test result. Optimal protocols are necessary to translate research sequences into clinical practice. Our aim was to evaluate the effect of the NDED on the performance of TBSS in a real world patient population. Before the TBSS approach enters the clinical routine, one needs to understand the effect of basic acquisition parameters on the results of the analysis. 


\section{Methods}

\section{Participants}

Thirty-nine MS patients and 34 healthy controls participated in study \#1 and 78 MS patients and 126 healthy individuals in study \#2. All the demographical data are in Table 2 . We applied the following inclusion criteria for MS patients in both studies: relapsing remitting form of the disease, taking disease modifying therapy, no relapse six months prior the measurements, EDSS score less than 6.5, no other major neurological, psychiatric and ophthalmological disease. We enrolled healthy controls who had no known neurological and psychiatric disease. Clinical data were collected for all the MS participants that is age, sex, EDSS, disease duration, and the used disease modifying therapy.

Study \#1 was carried out in accordance with the recommendations of the Medical Research Council National Scientific and Ethical Committee (ETT TUKEB) with written informed consent from all subjects. The protocol was approved by the National Institute of Pharmacy and Nutrition (000002/2016/OTIG). Study \#2 was approved by the local ethics committee (authority number: 56/2011). All subjects gave written informed consent in accordance with the Declaration of Helsinki.

\begin{tabular}{|l|l|l|l|l|l|}
\hline study & group & $\begin{array}{l}\text { number of } \\
\text { participants } \\
\text { (female) }\end{array}$ & age (year) & EDSS & $\begin{array}{l}\text { disease } \\
\text { duration } \\
\text { year) }\end{array}$ \\
\hline \multirow{2}{*}{ study \#1 } & MS & $39(25)$ & $39.1(+/-9.5)$ & $1.4(+/-1.4)$ & $8.6(+/-6)$ \\
\cline { 2 - 6 } & HC & $34(23)$ & $31(+/-10.9)$ & - & $8.7(+/-6.8)$ \\
\hline \multirow{2}{*}{ study \#2 } & MS & $78(55)$ & $38.6(+/-9.9)$ & $1.5(+/-1.6)$ & - \\
\cline { 2 - 6 } & HC & $126(57)$ & $31(+/-10.2)$ & - & - \\
\hline
\end{tabular}

Table 2 Demographical data of all subjects.

Study \#1

\section{Visuo-motor task}

The subjects completed a prosaccade and an antisaccade task. The investigation took place in a well-lit room. The subjects sat $60 \mathrm{~cm}$ away from the screen. The visual stimuli and the task paradigm were written using the Tobii MATLAB binding ${ }^{1}$ and the Psychophysics Toolbox Version 3.0.12², under MatLab 8.3.0.532 (2014a, MathWorks, Inc.). Eye movement recording was carried out with a Tobii TX300 eye tracker. Before the task, a 5 points calibration was carried out. The prosaccade task was the following: A black cross appeared in the center of a gray screen, which disappeared after a random interval of 1.2-2 seconds and appeared 
instantaneously in the left or right side of the screen, $9.2^{\circ}$ or $18.4^{\circ}$ from the center. Each condition (4 in all: left-far, left-close, right-far, right-close) was repeated 20 times in a pseudorandom order. Subjects had to move their gaze to the new location of the target instantly and accurately. Halfway during the task, there was a break to prevent subjects from fatigue and/or tearing. In the antisaccade task, the layout was the same, but the subjects had to move their gaze contralateral to the position of the new target.

The data acquisition started when the target (cross) jumped to the periphery and lasted one second. The sampling frequency was $300 \mathrm{~Hz}$. Data from both eyes were recorded simultaneously. Each recorded data point had a time stamp and a validity code. After the data acquisition, the target jumped back to the center of the screen.

\section{Data processing}

The recorded data was processed offline. Trials in which more than $10 \%$ of the data was missing (validity code higher than 1 as provided by the eye tracker manual) or more than $100 \mathrm{~ms}$ was missing continuously or more than $80 \%$ was missing in the first $50 \mathrm{~ms}$ were excluded from further analysis. In the rest of the trials, missing values were interpolated with linear interpolation of the neighboring values.

The pre-processed data were smoothed and differentiated with a 0,1 st and 2 nd order 11-points sliding window Savitzky-Golay filter ${ }^{1}$ to calculate the position, velocity and acceleration of the eyes. Saccades were detected automatically: if the velocity of the eye exceeded $50 \%$ in 2 consecutive points it was labelled as a saccade like event. The beginning of the saccade like event was marked where the acceleration of the eye was 0 (or reached its minimum value in $50 \mathrm{~ms}$ before its peak). The end of the saccade like event was marked where its velocity reached zero after the peak. Saccade like motion was accepted as a saccade if its latency occurred between $100 \mathrm{~ms}$ and $600 \mathrm{~ms}$ after stimulus onset, it took at least $12 \mathrm{~ms}$ and a fixation preceded the saccade like event. During fixation the eye had to be close to the initial cross (less than $1.5^{\circ}$ ) and its position change over the fixation had to be less than $0.6^{\circ}$. For all trials the position-time diagram was re-checked visually and inadequate trials were excluded. The first two trials in each condition were seen as practice and excluded from further analysis. A condition was accepted if the subject had at least 9 trials (half of the trials in a condition) after exclusion. Saccade latency (the start of the saccade), saccade peak velocity, saccade amplitude and saccade duration were assessed. Saccade gain was calculated from the ratio of 
the final eye position and the target position. Antisaccade latency, gain, peak velocity were determined similarly in the correctly performed trials. Antisaccade performance was calculated as the percentage of correctly performed trials to all the adequate trials. Moreover, a dysconjugacy index (DI) was calculated in the saccade task from both eyes as the ratio of the abducting and adducting eye's velocity in the "long" condition. DI was determined in the left and right directions. Patient's Z-scores were calculated as indicated in Equation 4.

$$
Z_{D I}=\frac{D I(\mathrm{MS})-\operatorname{mean}(D I(H C))}{S T D(D I(H C))}
$$

Equation 4

\section{Magnetic resonance imaging}

We used two scanners in the studies and protocols which we described in details in our recommendation ${ }^{58}$. In study \#1, magnetic resonance imaging was performed with a $3 \mathrm{~T}$ GE Discovery 750w MR Scanner (GE Healthcare, Chalfont St. Giles, UK). The following sequences were used in the current analysis: High resolution T1 weighted anatomical images (3D spoiled gradient echo images with inversion recovery (3D FSPGR IR: echo time [TE]: $2 \mathrm{~ms}$; repetition time [TR]: $5.4 \mathrm{~ms}$; inversion time [TI]: 450ms; matrix: $256 * 256$; field of view [FOV]: $25.6 \mathrm{~cm} * 25.6 \mathrm{~cm}$; flip angle: $12^{\circ}$; slice thickness [sl]: $1 \mathrm{~mm}$; PURE intensity correction), CUBE T2 FLAIR for lesion detection (TE: 135ms; TR: 6700ms; TI: 1827ms; matrix: 256*224; FOV: 25*22.5cm, sl: $1.4 \mathrm{~mm}$; fat sat; post processing: ZIP512, ZIP2), CUBE double inversion recovery (DIR) (TE: 90ms; TR: 7000ms; TI: 2901ms; blood suppression TI: 546ms; matrix: 192*192; FOV: $25 \mathrm{~cm} * 25 \mathrm{~cm}$; sl: 1.4mm; fat sat) and spin echo (SE) T1 weighted images (TE: min full, TR: 500, flip angle: $73^{\circ}$; matrix: 256*224, FOV: $24 \mathrm{~cm} * 19.2$, sl: 3mm, NEX: 2).

\section{Image analysis}

Lesion load was determined in the periventricular, infratentorial and juxtacortical regions on the FLAIR and DIR images manually. Lesion load in the whole brain as well as in the above-mentioned subregions were correlated with the behavior parameters. The SE T1 images were used to determine black hole burden.

The correlation of lesion location probability and eye movement deficit was evaluated as described by Kincses et al. ${ }^{59}$. Binary lesion masks were brought into standard space by registering the FLAIR images to the high resolution T1 weighted images by 6 DOF linear 
registration $^{60}$ and the $\mathrm{T} 1$ weighted images to standard MNI152 space by non-linear registration ${ }^{61}$. The standard space binary lesion masks were concatenated. A voxelwise GLM analysis was performed, the regressors of the design matrix were the measured eye movement parameters. Non-parametric permutation test, with 5000 permutations were used for statistical inference with correction for multiple comparisons.

The high resolution T1 weighted images were used for voxel-based morphometry analysis. We employed an "optimised" VBM-style protocol ${ }^{62,63}$ using FSL ${ }^{64}$. Non-brain parts were removed from all structural images ${ }^{65}$ and tissue-type segmentation was carried out by FAST $4{ }^{66}$. The resulting gray matter partial volume images were registered to standard space (MNI152) using linear transformation ${ }^{60}$ followed by a non-linear registration ${ }^{61}$. The resulting images were averaged to create a study-specific template, to which the native gray matter images were then non-linearly re-registered. The registered partial volume images were then modulated (to correct for local expansion or contraction) by dividing by the Jacobian of the warp field. The modulated segmented images were then smoothed with an isotropic Gaussian kernel with a sigma of $2 \mathrm{~mm}$. Finally, voxelwise GLM was applied and permutation-based nonparametric testing correcting for multiple comparisons across space was used for statistical inference. The design matrix contained the behavior parameters (saccade latency, peak velocity and gain) in consecutive analyses. The model was adjusted for disease duration and age. Thresholding was carried out by cluster-based thresholding corrected for multiple comparison by using cluster size.

\section{Statistical analysis}

Statistical analysis was carried out with Rstudio ${ }^{67}$. The following packages were used: lme $4^{68}$ - model building, car $^{69}$ - statistical significance. Mean and standard error were calculated for the following parameters: latency, peak velocity and gain from both eyes in the prosaccade and antisaccade tasks separately. All parameters were evaluated in a mixed model ANOVA, in which the subject was the random effect and the group (HC-MS), the movement type (abduction-adduction), and the distance (far-close) of the target handled as fixed effects. A p-value $<0.05$ was considered significant. We investigated both eyes separately because an average of the two eyes could be misleading if subclinical INO presents. The results from the left eye were reported unless otherwise stated. To investigate oculomotor decision, latency and peak velocity differences between prosaccade/antisaccade tasks were calculated and compared 
between the groups. The name of the new calculated variables were peak velocity difference and latency difference. As peak velocity and amplitude have linear relationship in case of significance the statistic was repeated with the scaled peak velocities. Pearson or Spearman correlation (where the assumptions of the Pearson were not valid) between MRI markers and behavior parameters were calculated in separate analyses. The effect of disease duration and age was tested in partial correlation and the effect of sex is tested via comparing the Fischer $\mathrm{Z}$ transformed correlation coefficients in the two sex separately. Correlation coefficient are reported from simple correlation where the age, disease duration and sex had no effect on the association.

Study \#2

\section{Magnetic resonance imaging}

In study \#2, imaging was performed on a 1.5 T GE Signa Excite HDxt MR Scanner (GE Healthcare, Chalfont St. Giles, UK). T1-weighted images (3D IR-FSPGR: TR/TE/TI: 10.3/4.2/450 ms, flip angle: $15^{\circ}$, ASSET: 2 , FOV: $25^{*} 25 \mathrm{~cm}$, matrix: $256^{*} 256$, slice thickness: $1 \mathrm{~mm}$ ) and 60 direction diffusion-weighted images with 6 non-diffusion-weighted reference volumes were recorded (TE: $93.8 \mathrm{~ms}$, TR: $13500 \mathrm{~ms}$, matrix: 96x96, FOV: 23x23 cm, flip angle: $90^{\circ}$, in-plane resolution: $2.4 \times 2.4 \mathrm{~mm}$, which was resampled to a $0.89 \times 0.89 \mathrm{~mm}$ by the scanner, slice thickness: $2.4 \mathrm{~mm}, \mathrm{~b}: 1000 \mathrm{~s} / \mathrm{m}^{2}$, NEX: 2, ASSET: 2).

\section{Image analysis and direction reduction}

The analyses of MR images were carried out with the tools from the FMRIB Software Library (FSL, version 5.0; Oxford Centre for Functional MRI of the Brain (FMRIB), UK;www.fmrib.ox.ac.uk/fsl) and in-house built MATLAB scripts. The following preprocessing methods were used in the diffusion image pipeline: eddy current correction and brain extraction. The FSL eddy_correct function was used to correct for subject movement and eddy currents and the FSL bet function in the non-diffusion weighted image to eliminate non brain part of the image.

From the original data (60 diffusion encoding directions) the diffusion encoding directions were reduced to $\mathrm{n}=10,15,20,25,30,35,40,45,50,55$ as follows. We used a reduction procedure to keep the uniform distribution via maximizing the total angular distribution energy. ${ }^{39}$ The angular distribution energy of a pair of points on the unit sphere surface could be determined as the inverse of the sum of the squares of the least spherical 
distance between point $a$ and point $b$, and the least spherical distance between point a and point b's antipodally symmetric point B (Equation 5)

$$
E_{a, b}=\frac{1}{\left(\min _{a, b} \operatorname{dist}_{a, b}\right)^{2}+\left(\min _{a, B} \operatorname{dist}_{a, B}\right)^{2}}
$$

The total angular distribution energy for a certain set of gradients can be calculated as the sum of the angular distribution energy of all pairs of gradients. The subset of gradient directions was chosen in a sequential order. ${ }^{39}$ The initial subset was one gradient and other gradients were chosen in a sequential order to maximize the total angular distribution of the set (Equation 6). The generated subset of diffusion encoding directions was selected and fed into the DTI analysis.

$$
E_{L}(N)=\sum_{a}^{N} \sum_{b}^{N} E_{a, b}
$$

Equation 6

We used the epi_reg function in FSL FLIRT ${ }^{70}$ to calculate the transformation matrix between the T1 image and diffusion space. A diffusion tensor model was fitted in each voxel with the diffusion toolbox of FSL. ${ }^{64}$ The fractional anisotropy maps were calculated in each group. We used FSL FAST ${ }^{66}$ on the T1 weighted images to segment the white matter from the high-resolution anatomical image. Next, the white matter tissue probability map was thresholded (0.7), binarized and transformed to the diffusion space with the inverse transformation matrix from epi_reg and a final threshold of 1 was used. All the individual white matter masks were visually inspected and in case of gross error the mask was recalculated and/or corrected manually. These individual white matter masks were used as region of interests (ROI) to evaluate parameters under the white matter area. The mean and standard deviation of FA, MD, axial diffusivity (first eigenvalue, AD) and radial diffusivity (mean of the second and third eigenvalue, RD) within the white matter mask were calculated for each subject, that is, one value was determined in every subsampled data set $(10,15,20,25,30,35,40,45,50,55$, 60 directions) for each subject. For every subject we calculated the bias from its original 60directions image for each subsampled data set, that is the 60 directions image was considered as a reference. We also tested for the statistical significance of the effect of directions on the mean and standard deviation for each derived parameters. Repeated measures of analysis of variance with the random effect of subjects was used within $\mathrm{R}$ studio ${ }^{67}$ as implemented in the 
lme4, emmeans and car packages. ${ }^{68,69,71}$ The FA, MD, RD and AD bias was also calculated within different white matter regions, by dividing the white matter mask into separate bins, namely areas with $0.2-0.4,0.4-0.6,0.6-0.8,0.8-1$ FA values in the reference (60-dir) image.

To evaluate the effects of the number of diffusion directions on TBSS results we performed a TBSS analysis separately on each DTI parameter as proposed in the FSL guideline. ${ }^{47}$ Briefly, subjects' FA images were aligned into a common space, using the nonlinear registration tool (FNIRT). A mean FA image was created and the threshold set at FA = 0.3 , deriving a mean FA skeleton that represented the centers of all tracts common to the group. Each subjects' aligned FA data were then projected onto this skeleton and the resulting data fed into voxel-wise statistics. We performed statistical analysis with the use of a general linear model with non-parametric permutation test for inference (5000 permutations) with age and sex as covariates in our model. Thresholding was performed by threshold free cluster enhancement approach and results were corrected for multiple comparisons by controlling for the familywise error rate. The analysis was performed in the following 4 subgroups of reduced directions: DTI parameter images of 15, 30, 45, 60 directions comparing the two groups (126 HC and 78 MS). In a second analysis, the threshold in the last step of the TBSS pipeline was chosen to keep skeleton size similar (voxel count difference within $0.1 \%$ ), therefore FA thresholds of $0.3225,0.307,0.3028,0.3$ were used for the $15,30,45$, and 60 directions, respectively. The number of voxels in the skeleton and the number of significant voxels were calculated. Also, the mean and standard deviation of the FA, MD, $\mathrm{RD}$ and $\mathrm{AD}$ values in the significant voxels were investigated. Mean group differences were calculated in the significant voxels. 


\section{Results}

\section{Oculomotor alterations in MS}

Clinical examination indicated that 5 patients (13\%) had clinically detectable oculomotor alteration. They were excluded from further quantitative analysis. One further patients was also excluded because of technical issues with the MRI images. The demographical data of subjects are presented in Table 1. All patients were on disease modifying therapy (6 patients take dimethyl-fumarate, 11-teriflonomide, 4- i.m. interferon beta1a, 7glatiramer acetate, 10-fingolimod, 1-s.c interferon beta1a). The average total lesion number was $21( \pm 15)$. As expected, most of the lesions occurred in the periventricular region (12.5 \pm 7.6$)$, but significant lesion load was found in the infratentorial and juxtacortical location too (1.5 $\pm 1.6,3.6 \pm 4.5$, respectively).

The latency of the anti-saccades were longer than the latency of saccades (194 ms vs. $303 \mathrm{~ms}, \mathrm{t}=-17.3, \mathrm{p}<0.0001$ for HCs and $207 \mathrm{~ms}$ vs. $319 \mathrm{~ms}, \mathrm{t}=-18.6, \mathrm{p}<0.0001$ for MS participants). Saccade latency was significantly prolonged in MS patients. The results were similar in both eyes [left: $\mathrm{F}(1,65.966)=5.36, \mathrm{p}=0.024$, right: $\mathrm{F}(1,65.98)=5.38, \mathrm{p}=0.024]$ [mean $( \pm$ sd): $194( \pm 24) \mathrm{ms}$ vs. $207( \pm 31) \mathrm{ms}$ for $\mathrm{HC}$ and MS participants]. There were no interaction effects between the fixed effects (group, movement type and distance of the target). The results are depicted in Figure 1. Anti-saccade latency was prolonged in MS patients, however it did not reach a significant level $[\mathrm{F}(1,64.96)=2.39, \mathrm{p}=0.12][\mathrm{mean}( \pm \mathrm{sd}): 303( \pm 44)$ ms vs. $319( \pm 44) \mathrm{ms}$ for HC and MS, respectively]. The latency difference between prosaccade

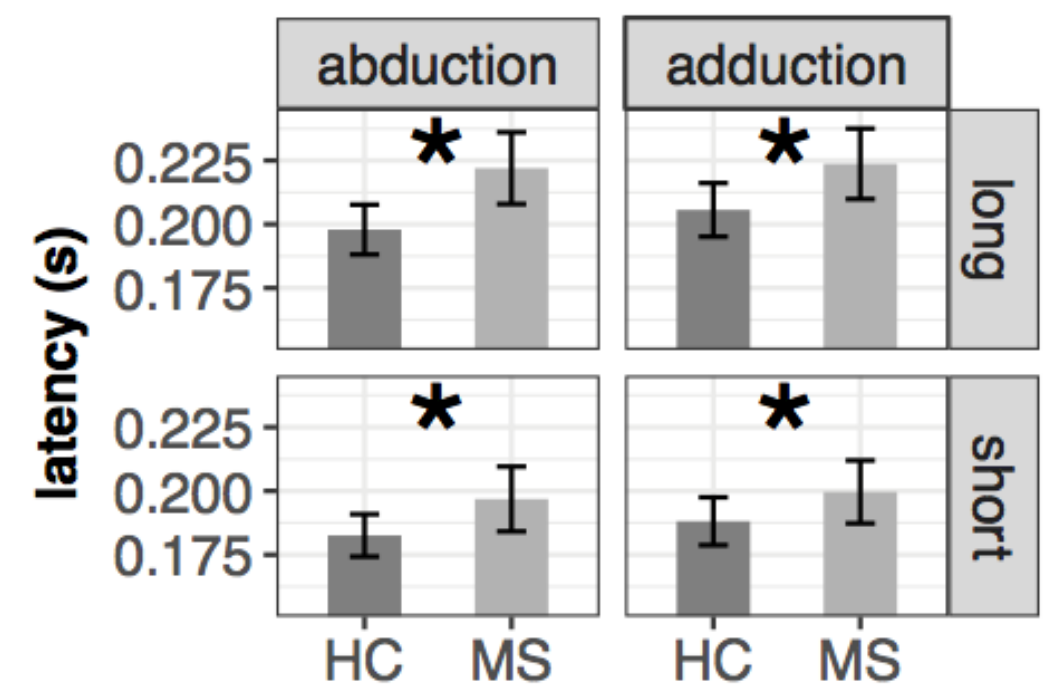

Figure 1 Mean and +/- SEM of latency in different conditions of the left eye in the saccade task. Significant difference in a group level could be observed. 
and anti-saccade tasks was also investigated on group level and we found no significant difference. $[\mathrm{t}(64)=0.29, \mathrm{p}=0.77][\mathrm{mean}( \pm \mathrm{sd}): 107( \pm 36) \mathrm{ms}$ vs. $112( \pm 34) \mathrm{ms}$ for HC and MS, respectively].

Prosaccade peak velocity was slightly smaller in the MS group [mean $( \pm \mathrm{sd}): 325( \pm 32)^{\circ}$ /s vs. $310( \pm 42)^{\circ} / \mathrm{s}$ for $\mathrm{HC}$ and MS, respectively] but there was no significant difference between the two groups $[\mathrm{F}(1,65.96)=1.4, \mathrm{p}=0.28]$. However, a significant interaction effect could be observed in two conditions: (i) group x movement type [left eye: $F(1,187.1)=3.3, p=0.071$, right eye: $\mathrm{F}(1,192.7)=7.2, \mathrm{p}=0.0079]$, which means that the higher velocity of adduction compared to abduction in $\mathrm{HC}$ was reversed in MS patients, resulted in a higher peak velocity in abduction compared to adduction (Figure 2) and (ii) group x distance left eye: $\mathrm{F}(1,187.1)=$ 7.7, $\mathrm{p}=0.006$, right eye: $\mathrm{F}(1,192.2)=7.15, \mathrm{p}=0.008$ ], which means that the slower peak velocity in the closer cue condition in HC group was slightly smaller in the MS group. Antisaccade peak velocity was not different between the two groups $[F(1,64.8)=0.12, p=0.73]$

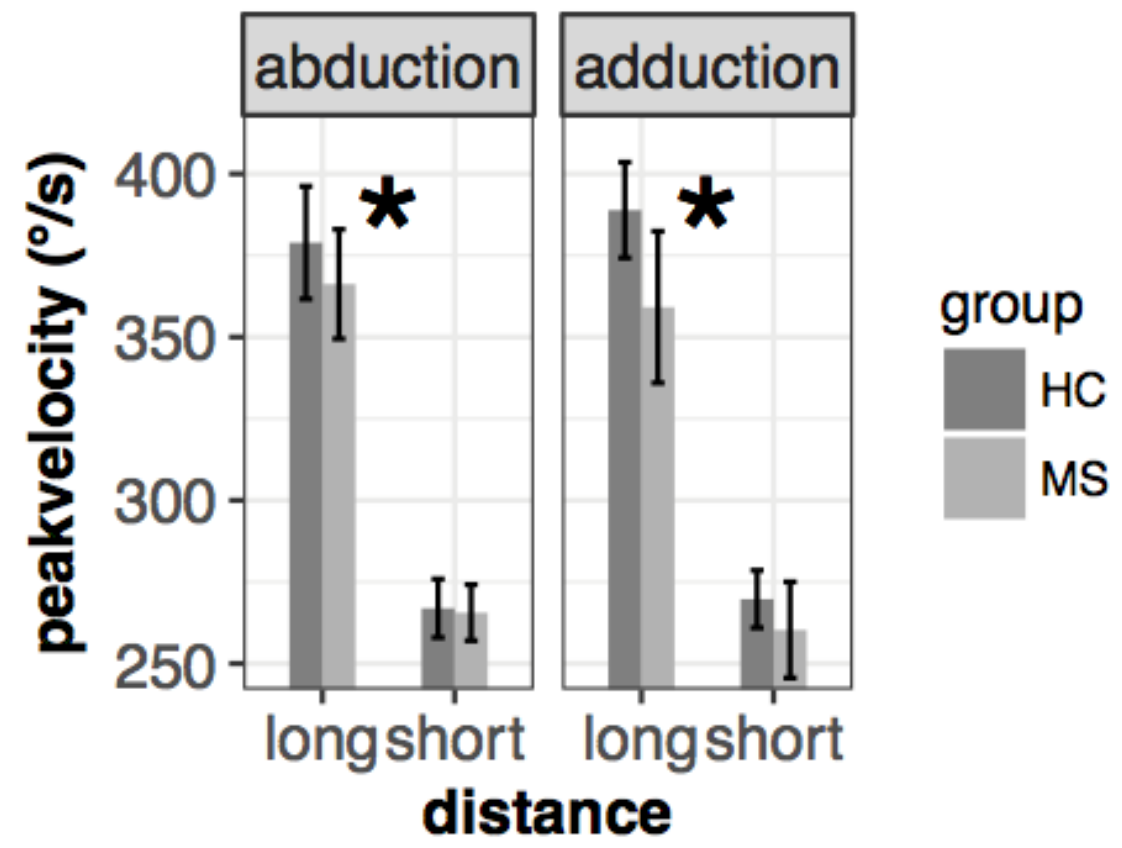

Figure 2. Mean and +/- SEM of peak velocity in different conditions of the left eye in the saccade task. Significant group*distance interaction effect could be observed.

$\left[\right.$ mean $( \pm \mathrm{sd}): 265( \pm 46)^{\circ} / \mathrm{s}$ vs. $268( \pm 41)^{\circ} / \mathrm{s}$ for $\mathrm{HC}$ and MS, respectively]and no interaction effects could be observed. Difference between prosaccade and anti-saccade peak velocity could be observed in a group level. MS group had lower difference in peak velocity around 20/s [t(64) $=2.13, \mathrm{p}=0.037][\mathrm{mean}( \pm \mathrm{sd}): 60( \pm 33) \circ / \mathrm{s}$ vs. $42( \pm 37) \circ / \mathrm{s}$ for $\mathrm{HC}$ and MS, respectively $]$, however, this difference was not survived scaling for amplitude [t(64) $=0.07, \mathrm{p}=0.95$ ] 
Moreover, EDSS scores positively correlated with peak velocity difference (Spearman's rho: $0.4, p=0.024)$. The higher clinical disability related to higher peak velocity difference between the two tasks.

In the prosaccade task, MS group had smaller gain in all conditions. Therefore, they performed slightly hypometric saccades compared to HC. However, this difference did not reach a significant level $[\mathrm{F}(1,65.8)=2.24, \mathrm{p}=0.14][\mathrm{mean}( \pm \mathrm{sd})$ : $0.932( \pm 0.046)$ vs. 0.913( \pm 0.049$)$ for $\mathrm{HC}$ and MS, respectively]. Gain in the anti-saccade task did not differ between the two groups $[\mathrm{F}(1,63.6)=0.01, \mathrm{p}=0.92]$.

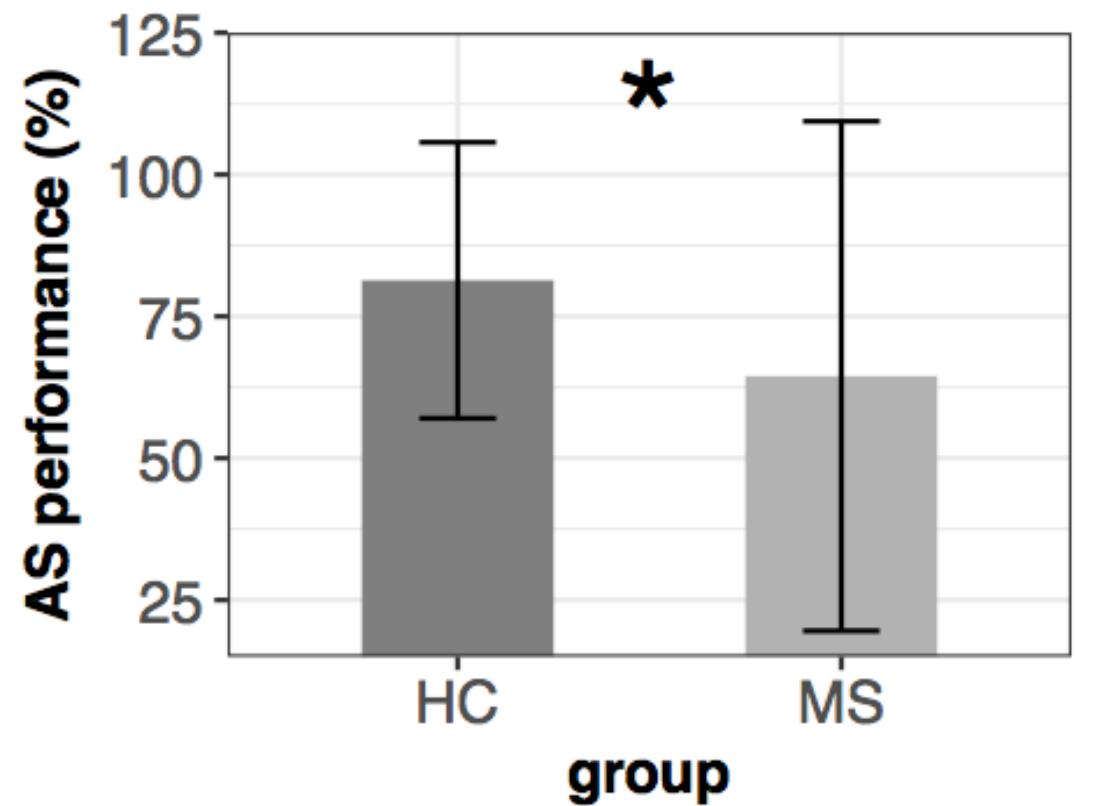

Figure 3. Mean +/- SEM of antisaccade performance in the two groups.

There was a marked difference between the two groups in the anti-saccade performance. The HC group reached more than $80 \%( \pm 12.2 \%)$ accuracy while the MS group obtained only 64\% ( $\pm 22.5 \%$ ). (Wilcoxon-Mann-Whitney test: [U34, $33=837, \mathrm{p}<0.001]$ (Figure 3).

The dysconjugacy index derived from peak velocities of the eyes can detect clinically not detectable INO. A Z-value higher than the highest control subject's z-value+2 was determined as threshold for subclinical $\mathrm{INO}^{56}$. Based on this threshold five patients were classified as having subclinical INO. Patient \#49 had INO in both directions, while patient \#39, $\# 37$, and \#17 had only in the left direction and patient \#18 only in the right direction (Figure 4).

T2 lesion burden or lesion location did not show significant correlation with any of the measured MRI parameters. A positive correlation were detected between anti-saccade latency and the number of black-holes (Spearman's rho: $0.45, \mathrm{p}=0.011$ ) and negative correlation between the anti-saccade peak velocity and the number of black-holes (Spearman's rho: -0.47 , 
$\mathrm{p}<0.01$ ) (Figure 5). There were no significant correlation between white matter volume with any of the measured eye movement parameters. The VBM analysis revealed that anti-saccade peak velocity correlated with gray matter density in parietal areas (Figure 6). That is, smaller anti-saccade peak velocity was associated with lower gray matter densities in the left parietal areas. No other eye movement parameters showed correlation with gray matter density

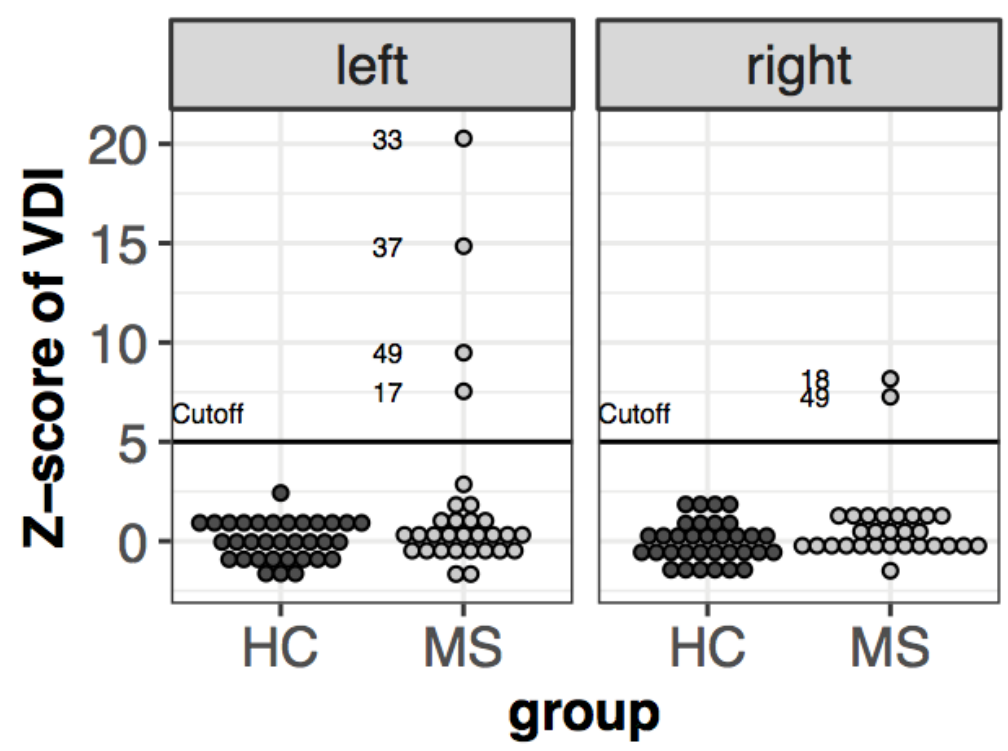

Figure 4 Z-scores of velocity dysconjugacy index individually, Subjects'value higher than the cutoff are labeled.

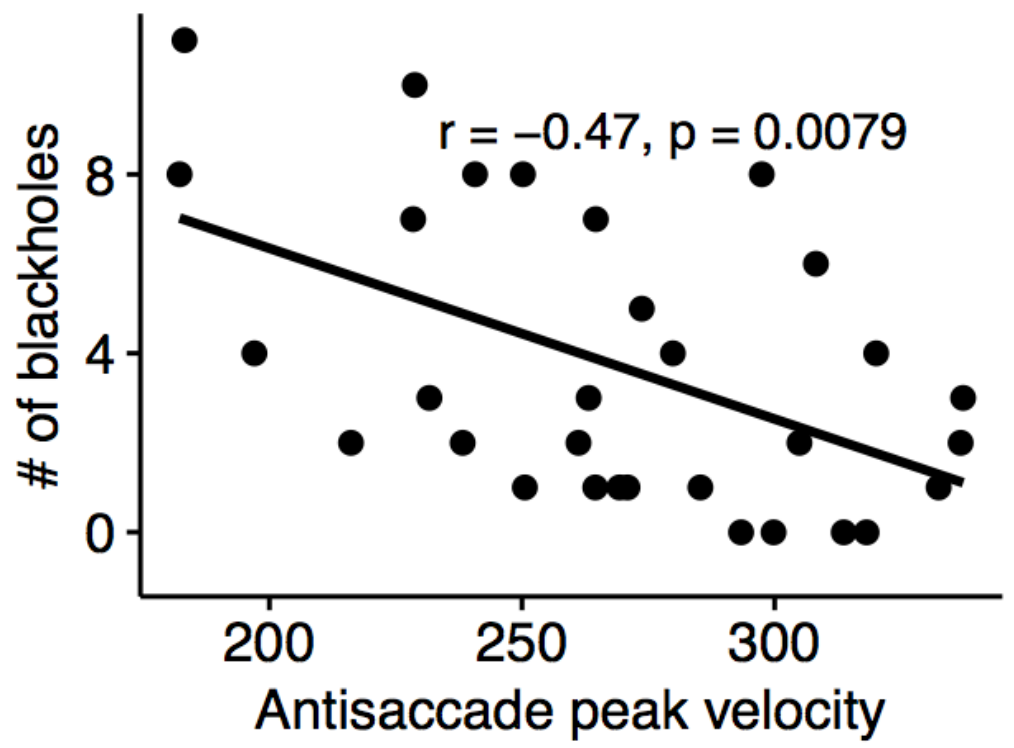

Figure 5 Antisaccade peak velocity of the left eye negatively correlated with blackhole count. 

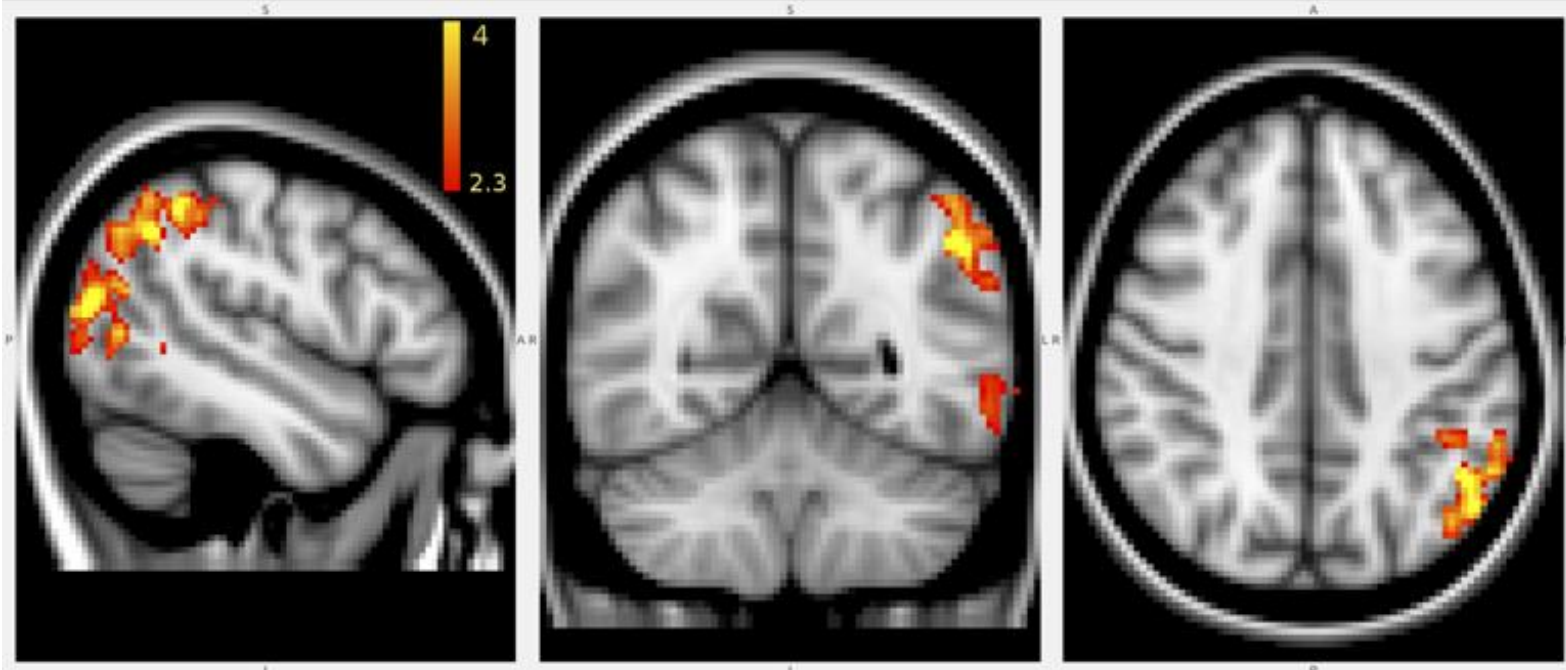

Figure 6 Results of VBM analysis. Marked voxels positively correlated with antisaccade peak velocity. The error bar represents different Z-value after cluster based thresholding. Disease duration and age were used as cofounders.

Effects of NDED on TBSS analysis

We considered the value of the '60 directions' group as the reference value. The NDED related mean DTI parameters are listed in Table 3 and bias is depicted in Figure 7. A decreasing
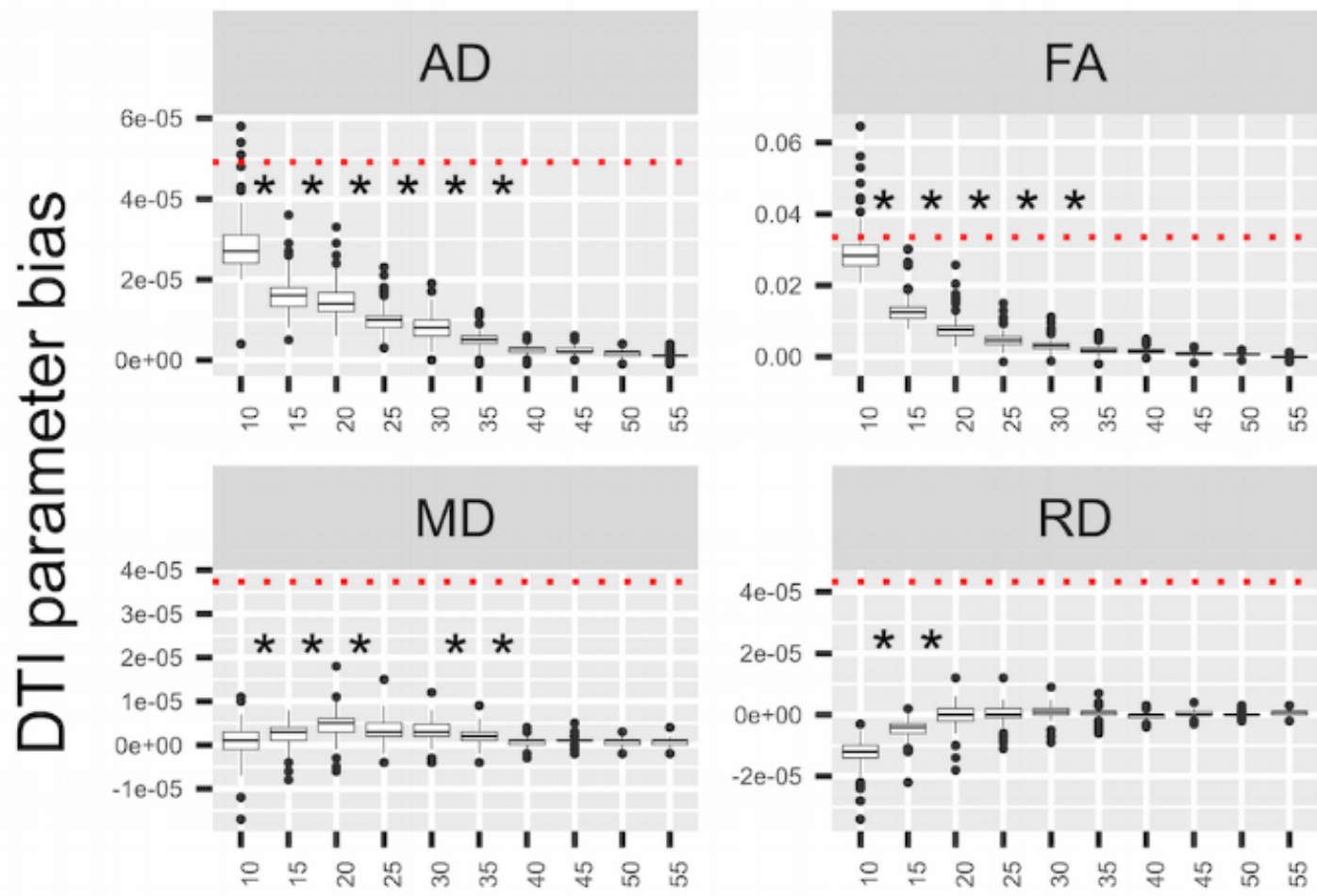

\section{NDED}

Figure 7 Bias of DTI parameters in white matter. The mean of DTI parameters' bias values under the white matter at different number of diffusion encoding directions in the HC group. The red dotted line shows the mean difference between the MS and HC group. Asterisks represent significant differences between groups with different directions.

trend of $\mathrm{AD}$ and FA could be observed with increasing number of diffusion encoding directions 
(Tables 3). The RD had an increasing trend and the MD was constant (Table 3 and Figure 7). Statistical analysis revealed significant main effect of directions in FA $(F(10,1250)=2191.8$, $\mathrm{p}<0.0001), \mathrm{MD}(\mathrm{F}(10,1250)=114.4, \mathrm{p}<0.0001), \mathrm{AD}(\mathrm{F}(10,1250)=1472.6, \mathrm{p}<0.0001)$ and $\mathrm{RD}$ $(\mathrm{F}(10,1250)=602.1, \mathrm{p}<0.0001)$. Moreover, the 40, 35, 35, 20 directions did not significantly differ from the 60 directions based on post-hoc analysis (Tukey method) in AD, FA, MD and RD, respectively (Figure 7). The asterisk in Figure 7 depicts NDED groups which differ significantly from the 60 directions group (Figure 7).

\begin{tabular}{|r|l|l|l|l|}
\hline NDED & AD & FA & MD & RD \\
\hline 10 & $1.20 \mathrm{e}-03$ & $4.86 \mathrm{e}-01$ & $7.58 \mathrm{e}-04$ & $5.39 \mathrm{e}-04$ \\
\hline 15 & $1.18 \mathrm{e}-03$ & $4.69 \mathrm{e}-01$ & $7.60 \mathrm{e}-04$ & $5.48 \mathrm{e}-04$ \\
\hline 20 & $1.18 \mathrm{e}-03$ & $4.64 \mathrm{e}-01$ & $7.62 \mathrm{e}-04$ & $5.52 \mathrm{e}-04$ \\
\hline 25 & $1.18 \mathrm{e}-03$ & $4.61 \mathrm{e}-01$ & $7.61 \mathrm{e}-04$ & $5.52 \mathrm{e}-04$ \\
\hline 30 & $1.18 \mathrm{e}-03$ & $4.59 \mathrm{e}-01$ & $7.61 \mathrm{e}-04$ & $5.53 \mathrm{e}-04$ \\
\hline 35 & $1.17 \mathrm{e}-03$ & $4.58 \mathrm{e}-01$ & $7.59 \mathrm{e}-04$ & $5.53 \mathrm{e}-04$ \\
\hline 40 & $1.17 \mathrm{e}-03$ & $4.58 \mathrm{e}-01$ & $7.58 \mathrm{e}-04$ & $5.52 \mathrm{e}-04$ \\
\hline 45 & $1.17 \mathrm{e}-03$ & $4.57 \mathrm{e}-01$ & $7.58 \mathrm{e}-04$ & $5.52 \mathrm{e}-04$ \\
\hline 50 & $1.17 \mathrm{e}-03$ & $4.57 \mathrm{e}-01$ & $7.58 \mathrm{e}-04$ & $5.52 \mathrm{e}-04$ \\
\hline 55 & $1.17 \mathrm{e}-03$ & $4.56 \mathrm{e}-01$ & $7.58 \mathrm{e}-04$ & $5.53 \mathrm{e}-04$ \\
\hline 60 & $1.17 \mathrm{e}-03$ & $4.56 \mathrm{e}-01$ & $7.57 \mathrm{e}-04$ & $5.52 \mathrm{e}-04$ \\
\hline
\end{tabular}

Table 3 The mean of DTI parameters in the individual white matter mask for different subset of encoding directions for healthy controls. $A D$ - axial diffusivity, $F A$ - fractional anisotropy, $M D$ - mean diffusivity, $R D$ - radial diffusivity, NDED - number of diffusion encoding directions

Mean values of DTI parameters under different bins of the white matter were evaluated (Figure 8). Lower FA bins had a greater overestimation with decreasing directions and this effect was not seen in MD. The standard deviation of DTI parameters in the white matter mask increased slightly with decreasing directions, which was more visible for FA and AD parameters. For the different intensity-bins, the FA value had the lowest standard deviation in the highest bin compared to other bins. In RD and MD parameters, the three upper bins had similar but lower standard deviation compared to the lowest bin. In contrary, the highest bin had the highest standard deviation compared to other bins in AD. As a function of NDED, the standard deviation of the whole white matter did not change significantly from the 60 directions at 55, 20, 35 and 20 directions in $\mathrm{AD}, \mathrm{FA}, \mathrm{MD}, \mathrm{RD}$ respectively as revealed with post-hoc analysis (Tukey method). 
$\mathrm{AD}$

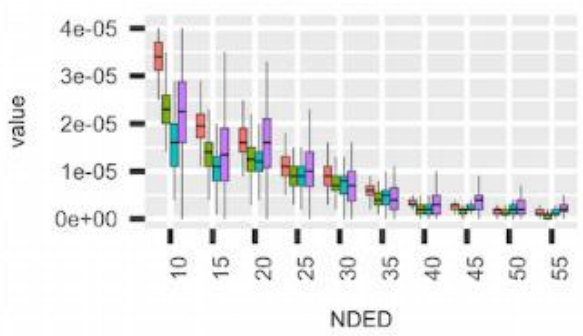

MD

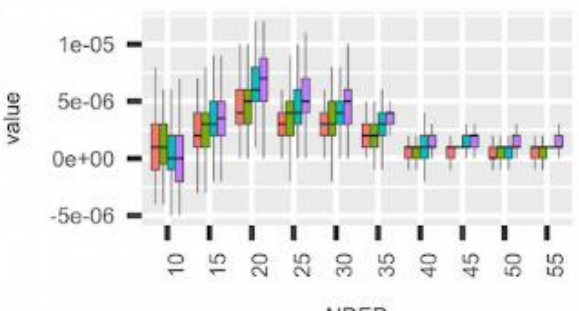

FA

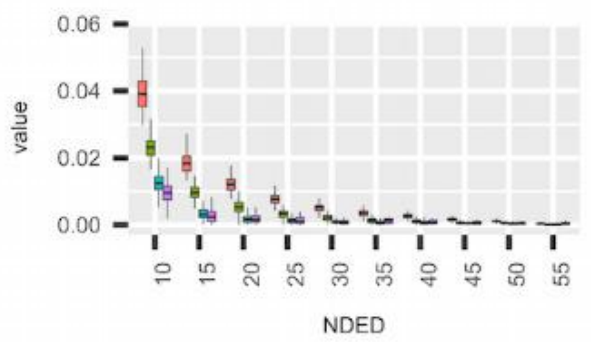

RD

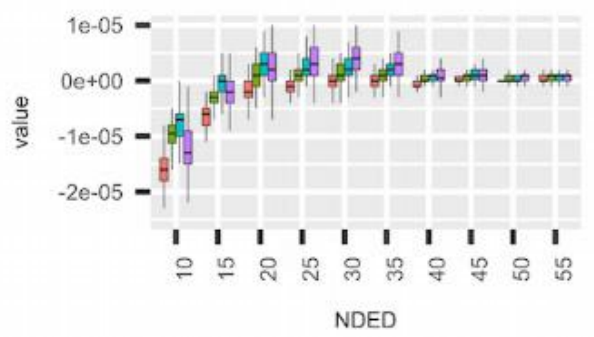

Figure 8 Bias of DTI parameters in different FA bins. The mean DTI parameter's bias from the "60 directions" image under areas of different FA values in the HC group. The four investigated DTI parameter are shown. Data points, which are out from the $95 \%$ interval, are not visualized.

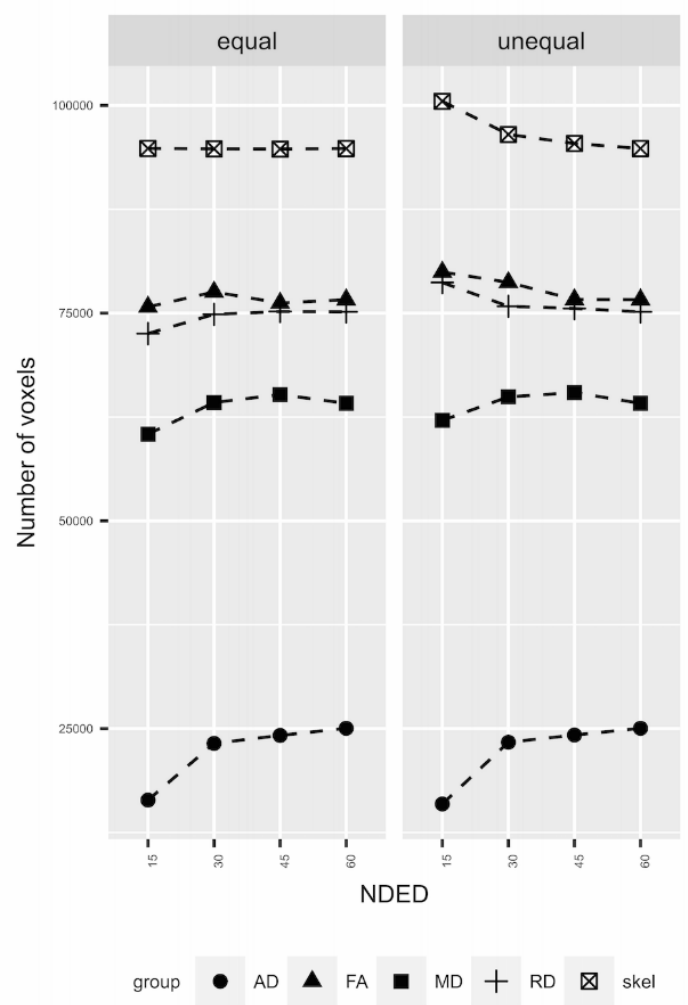

Figure 9 The results of TBSS analysis. The left side of the plot ("equal") shows the results of TBSS when skeleton size was held constant (see Methods). The right side of the plot ("unequal") shows the results when skeleton size was varying with $N D E D$. The different groups represent the significant voxel count in that diffusion parameter. Correcting for skeleton size the number of significant voxel count did not change in FA and RD as a function of NDED and the trend of AD and MD parameter were the same.

The TBSS analysis between 78 MS patients and 126 healthy controls revealed slightly 
different results as a function of NDED when threshold values were kept constant. The qualitative analysis revealed minimal differences in the skeleton size and the number of

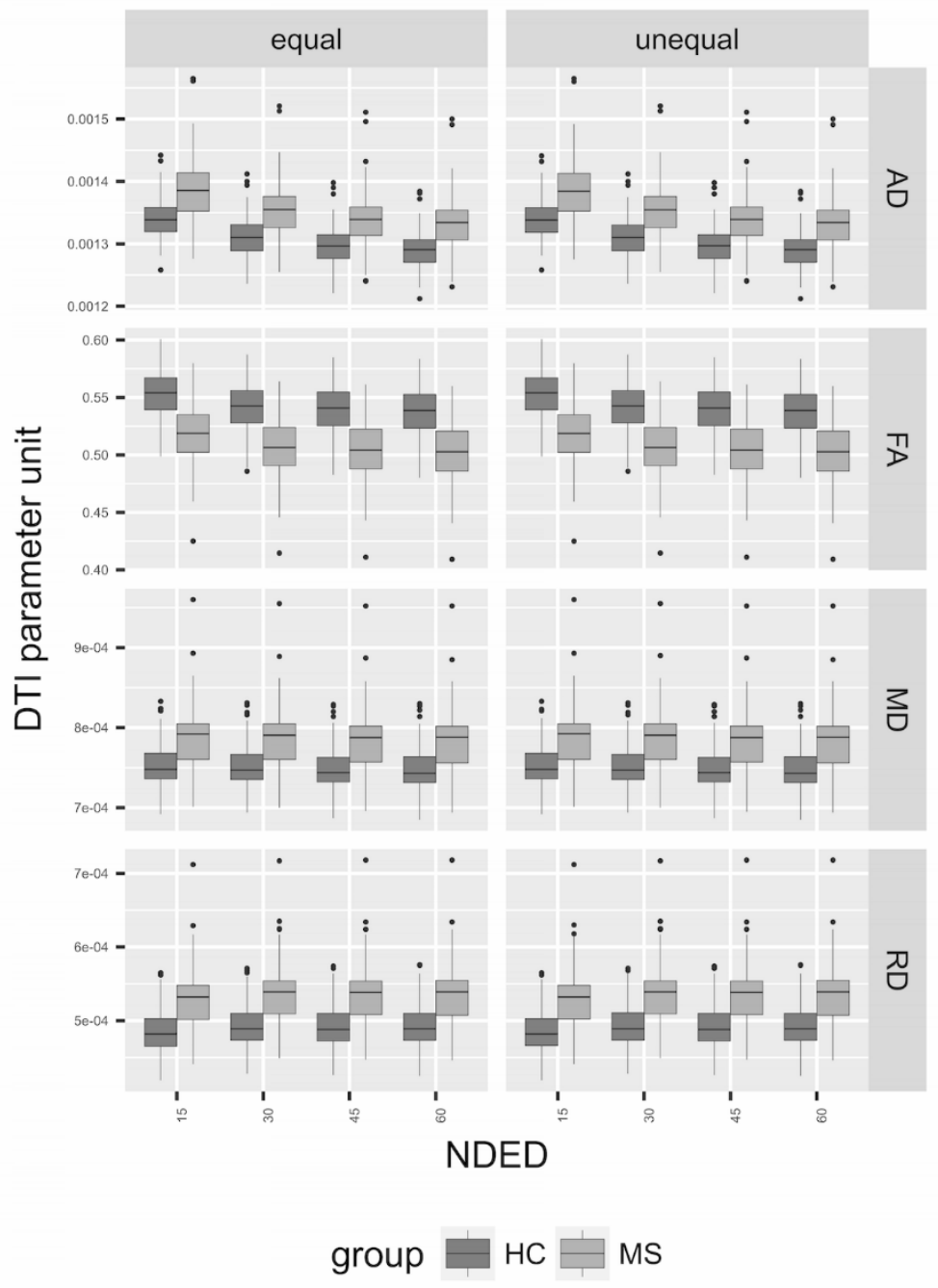

Figure 10 The mean FA under significant voxels. The left side of the plot ("equal") shows the distribution of the mean of DTI parameters under significant voxels when skeleton size was held constant (see Methods). The right side of the plot("unequal") shows the same results when skeleton size was varying with NDED.

significant voxels (Figure 9 - right side). The total size of the skeleton was reduced with increasing number of diffusion directions. Similarly, the number of significantly different voxels was reduced. However, when the FA threshold was changed to keep the skeleton size constant the number of significant voxels was similar in FA and RD but there was a drop at 15 directions in MD and AD (Figure 9. - left side). Regarding the group differences, the mean FA value within the significant voxels of the skeleton showed a decreasing trend with the elevation of the encoding directions. This was more pronounced in AD but absent in MD and RD (Figure 9-10.). In addition, the MS group had smaller values in any number of diffusion directions for 
FA and higher values for $\mathrm{AD}, \mathrm{MD}$ and $\mathrm{RD}$ on a group level (Figure 10). There was a statistical significant interaction between the effect of disease group and NDED in the equal skeleton size condition for all parameters $(\mathrm{AD}: \mathrm{F}(3,606)=10.2$, $\mathrm{p}<0.0001$; $\mathrm{FA}: \mathrm{F}(3,606)=8.4, \mathrm{p}<0.001$; $\mathrm{MD}$ $\mathrm{F}(3,606)=2.9, \mathrm{p}<0.05) ; \mathrm{RD}(\mathrm{F}(3,606)=4.2, \mathrm{p}<0.01)$. In the unequal skeleton size condition, interaction could be observed for $\mathrm{AD}, \mathrm{FA}, \mathrm{MD}$ and $\mathrm{RD}$ as well. $(\mathrm{AD}: \mathrm{F}(3,606)=10.2$, $\mathrm{p}<0.0001$; FA: $F(3,606)=8.8, \mathrm{p}<0.0001$; $\mathrm{MD}: \mathrm{F}(3,606)=2.7, \mathrm{p}<0.05)$; $\mathrm{RD}:(\mathrm{F}(3,606)=4.8, \mathrm{p}<0.01)$. This means that the mean differences of the groups change differently as a function of direction in both skeleton sizes. The result of the TBSS analysis of the FA in unequal skeleton size condition is depicted in Figure 11. 


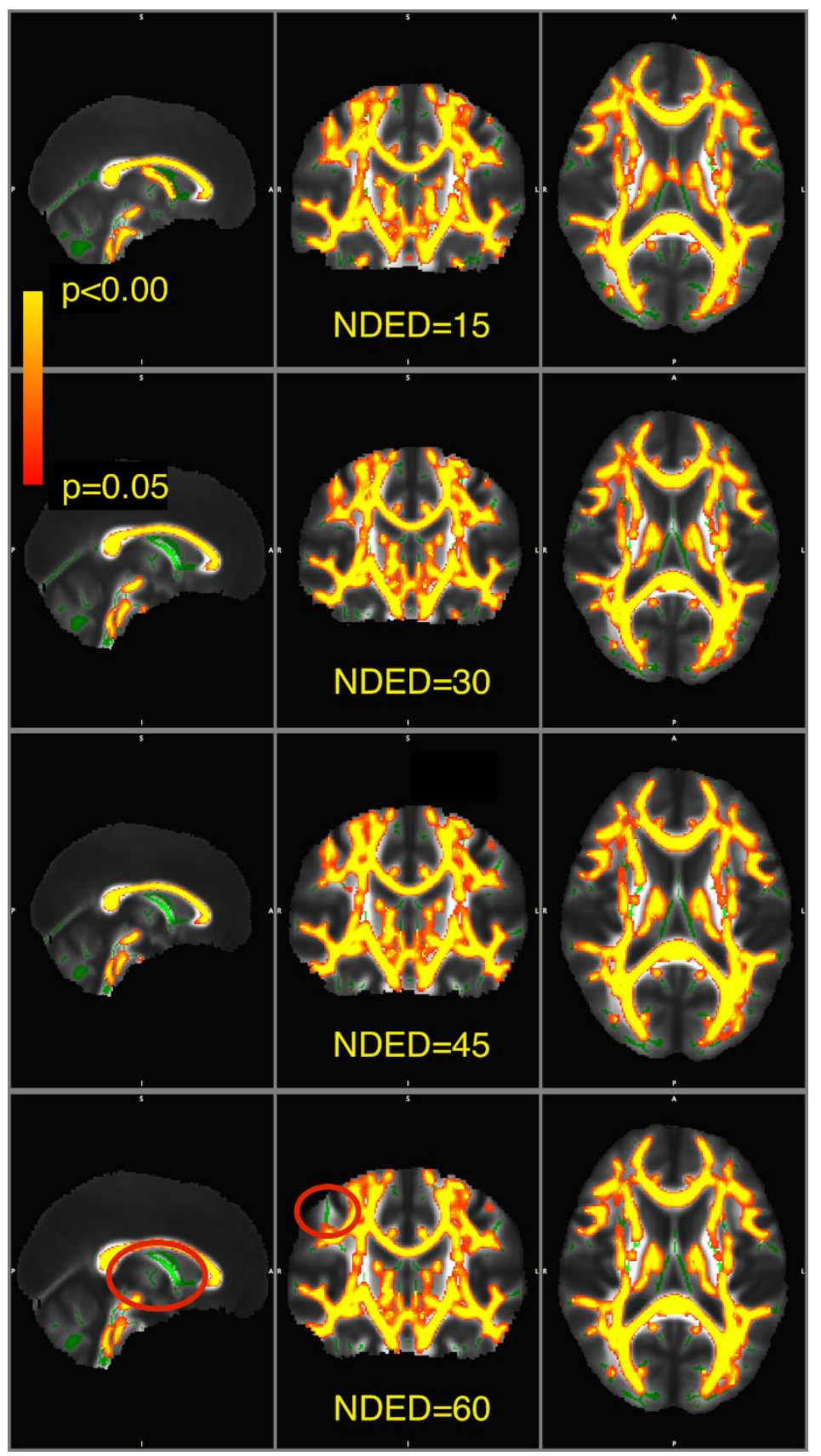

Figure 11 Visualization of significant voxels within the skeleton when skeleton size was unequal. The skeleton is showed in green. The statistical images are overlaid in yellow-to-red and thickened with the tbss_fill function for visualization as recommended in FSL. The yellow-to-red colours represent $p$-values thresholded at $p<0.05$ corrected. Red ovals show areas where significant voxels differ as a function of NDED. 


\section{Discussion}

In our study we investigated visually guided prosaccade and antisaccade task performance in MS patients and their possible association with focal brain alterations. Out of several eye movement parameters, we found significantly increased latency in the prosaccade task and significantly worse performance in the antisaccade task in MS patients. The detailed examination of conjugated eye movements revealed 5 subclinical INO cases.

As regarding the MRI parameters, the peak velocity and latency of the antisaccade movement correlated with the number of black-holes, but none of the eye movement parameters were associated with the T2 lesion burden or location. Most importantly, local gray matter atrophy in the left inferio-parietal lobule and temporo-parietal junction correlated with antisaccade peak velocity.

Oculomotor alterations found with various paradigms such as visually guided saccade, prosaccade, antisaccade, memory guided saccade and endogenous cued saccade are common in $\mathrm{MS}^{48,50,79,56,72-78}$. In agreement with our findings, Clough and coworkers found that saccade latency is prolonged in clinically definitive MS patients ${ }^{72}$. In the same cohort, latency increases with longer disease duration. In another study, the MS group has longer saccade latency in the presence of a distractor stimulus ${ }^{77}$. Previous studies also showed that the performance is deteriorated mainly in more cognitively demanding saccade tasks ${ }^{50,72-76,79}$. Antisaccade performance is deteriorated and associated with cognitive performance ${ }^{72,76,79}$. Fielding claims that this alteration spares the reflexive part of the saccades. In our investigation the antisaccade peak velocity, but not that of the parameters of the saccade task correlated with number of black-holes and focal gray matter atrophy in the temporo-parietal region. Prolonged latency of prosaccade could mirror the delayed initiation of saccades. The prolonged latency of antisaccades however, might reflect a prolonged volitional decision process or a delayed initiation of saccade in the opposite direction or both ${ }^{80}$. The difference might relates to the time, which is not necessary for the reflexive part such as inhibition or vector transformation ${ }^{81}$. Hence the correlations we have found are mainly reflecting the higher order cognitive processes of eye movements rather the reflexive parts. Interestingly, no correlation was found between any of the MRI parameters and the antisaccade performance. The non-reflexive part of the antisaccades might be dysfunctional, leading to an error. While if it is delayed but to a level that is not sufficient to make an error it could only be investigated via its delayed latency. This could 
especially be important in multiple sclerosis, in which demyelination, and slowed conduction is a key feature of the disease.

Saccades could be a potential marker to follow-up cognitive alterations in MS patients because it has been shown that various saccade performances are associated with $\operatorname{cognition}^{72,73,75}$. Based on these observations, prolonged latency could reflect damage to networks associated with motor or cognitive control. Eye movement could be a potential marker to test the integrity of networks involved in cognition because their cortical, subcortical and cerebellar networks overlap.

While several studies showed alterations of oculomotor performance in MS, the background of such alterations is not entirely clear. Clinically detectable oculomotor symptoms show correlation with certain infratentorial lesions ${ }^{3}$, but in our investigation, subclinical eye movement deficit did not correlate with T2 hyperintense lesion load, which is congruent with the result of a previous study ${ }^{79}$. It is in agreement with our earlier investigation in that lesion load or location only modestly correlate with clinical symptoms ${ }^{59}$, whereas persistent blackholes show better correlation with clinical and cognitive functioning in MS ${ }^{19,82}$. Accordingly, the T1 hypointense lesion burden correlated with the antisaccade velocity and latency in our study. Brain atrophy shows better correlation with clinical and cognitive disability ${ }^{83}$. In particular, cerebellar atrophy is associated with antisaccade error ${ }^{79}$. In our study, gray matter atrophy measures correlated with eye movement deficits globally as well as locally. Black holes and atrophy seem to jointly relate to antisaccade alterations. Several studies found correlation between T1 black-hole lesions and atrophy, but no similar relationship was revealed for T2 hyperintense lesions ${ }^{23,84}$. Cellular damage could be observed in both cases ${ }^{21,85}$. In addition, both measures correlate well with clinical disability, better than T2 lesion load ${ }^{86}$.

Saccade peak velocity is affected by multiple cognitive functions (arousal ${ }^{87}$ and mental workload $^{88}$ ). In our study, focal gray matter volume variability showed correlation with antisaccade peak velocity in the left inferior parietal lobule, left temporo-parietal junction and in the putative left V5/MT motion sensitive visual region ${ }^{89}$. These parietal regions are identical to those frequently implicated in attention tasks ${ }^{90}$. In their seminal paper, Corbetta and coworkers in a remarkably similar paradigm found activation in the intraparietal sulcus during sustained attention and in the right temporo-parietal junction when a target was detected, particularly at an unattended location ${ }^{51}$. These two conditions correspond to the top-down and bottom-up attentional subsystems. Moreover, the parietal cortex has its direct connection to the 
superior colliculus $^{53-55}$, and the pontine nuclei as well ${ }^{91}$. Damage to these perisylvian regions was also implicated in neglect ${ }^{92}$. Visuospatial neglect in MS patients has been described, but no associated structural damage has been found so $\mathrm{far}^{93}$. Interestingly, the right temporoparietal junction is implicated in target detection, but in our study the atrophy of the left temporo-parietal junction was associated.

Alternatively, parietal region has a potential role in saccades. The parietal eye field and posterior parietal cortex are involved in saccade generation ${ }^{94}$ and visuospatial attention ${ }^{90}$. Moreover, human ${ }^{95}$ and animal ${ }^{96}$ studies suggested that this region has a role in the vector inversion process, which is a crucial step in antisaccade.

The investigation of eye movement aids the diagnosis or the quantification of disease severity. While bedside oculomotor investigation could miss subtle alterations, eye tracker devices could be more precise and semi-automatically evaluate the records. Therefore, they could be useful to monitor disease progression. Subclinical disease progression could be screened on a regular basis.

We found gray matter alterations in association with oculomotor deficits. In addition, white matter impairment relates to antisaccade performance ${ }^{79}$. The joint damage of gray and white matter explain cognitive disturbance in $\mathrm{MS}^{97}$, which is in close relationship with eye movements ${ }^{75}$. To further elucidate the structural background of the observed oculomotor alterations, white matter microstructure should be investigated. The optimal choice of diffusion tensor imaging parameters is crucial to spare scan time but detect pathological alterations. As NDED is directly proportional to scan time, we investigated its effects on detection of disease related alterations. Microstructural alteration are described in MS. Demyelination could be in the background of the widespread changes in the main white matter tracts. The exact difference from the healthy controls could depend on the used measurement parameters such as the NDED. We investigated the effect of the number of diffusion directions on the estimated diffusion parameters, with a special emphasis on detecting differences in a patient population with the commonly used TBSS approach. We found an overestimation of FA and AD with 0.01 and $10^{-}$ ${ }^{5}$, respectively, if the NDED was below 30 directions. Areas with low FA values seemed to be more prone to the overestimation. For RD underestimation was found and MD was not affected by the NDED. When considering the TBSS analysis, the overestimation of FA leads to an enlarged skeleton and within that skeleton more significantly different voxels can be found at low NDED. 
At least six non-collinear encoding directions are necessary to estimate the diffusion tensor. ${ }^{98}$ Moreover, previous studies with real-life data ${ }^{39,42,43,99}$ investigated the effects on NDED on accurate and precise FA estimation in ROI analysis. The decrease of the encoding directions leads to an overestimation of the FA and $\mathrm{AD}$ and an underestimation of RD but does not affect MD. The overestimation seems to be more severe if the uniform spherical distribution of directions has not been kept. ${ }^{99}$ As it was emphasized, the relationship of the fiber orientation and the encoding direction has a major effect on the tensor, especially in low NDEDs. Simulation studies found that the relationship of diffusion scheme and the underlying fiber orientation has a profound effect on the accuracy and precision of the estimated anisotropy. ${ }^{41}$ However, the investigation of the performance of different schemes on real-life data is more complicated because of the spatial and tissue dependent SNR and other imaging related artifacts such as chemical shift, distortion and eddy currents.

The optimal NDED has been investigated in previous studies. ${ }^{39,42,43,99}$ In a ROI based approach, no difference was found in FA and MD between 6 and 30 directions when scanning time was held constant at $1.5 \mathrm{~T}{ }^{43}$ Moreover, they found an overestimation of the main eigenvalue $(\mathrm{AD})$ in the 6 directions scheme in most of the ROIs. Another ROI approach found minimal but significant FA and MD changes between the 6 and 30 directions scheme at $1.5 \mathrm{~T} .{ }^{42}$ However, they concluded that the test-retest variability is higher than this difference. In addition, an upward bias of FA could be detected in the gray matter as SNR decreased but no change was found in white matter ROIs. ${ }^{100}$ The total number of scans was held constant for these studies, which resulted in a comparable SNR for the different schemes. Moreover, an increment in NDED was reported to lead to an elevation in SNR and to reach its plateau at 53 directions for FA and 51 directions for MD in the white matter. ${ }^{39}$ Random rejection of directions leads to an overestimation of FA, AD and an underestimation of RD but does not affect MD, these effects are more apparent in areas with low FA. ${ }^{99}$ The uniform rejection has the same effects on low FA areas, but the bias is much smaller. Lower SNR could be in the background of the overestimation with lower NDED. Moreover, FA and AD seem to be affected more profoundly by NDED in general than "isotropic indices" (MD, RD) which averages information from more directions. The previously investigated ROIs mostly contain main tracts in which axons run in one direction. Nevertheless, the relative orientation of tracts and diffusion directions has an impact on the parameter estimation at low number of directions. ${ }^{41,42}$ However, 
the analyses of DTI data mostly use the full brain and TBSS is one popular method to do this. ${ }^{47}$ Therefore, to understand the effects of NDED on TBSS analysis is crucial.

Diffusion parameters have a high spatial variability (e.g. FA in the middle of the fibre bundle is much higher than at the periphery, or for example over the antero-posterior aspect of the corpus callosum ${ }^{101} \mathrm{FA}$ changes together with the underlying histological features). This high spatial variability warrants voxel wise statistics. However, registration in the white matter is not trivial. One possible solution is applied in the TBSS approach, namely only the most structured parts of the white matter are investigated in the white matter skeleton. The skeleton is defined by the relatively high FA values (usually higher than $0.2-0.3$ ), which by itself reduces the bias, since areas with low FA values are more prone to the overestimation of FA.

Multiple sclerosis is an inflammatory and neurodegenerative disease with various clinical symptoms in which white and gray matter are affected diffusely. ${ }^{102-104}$ The disease causes local lesions in the brain and alterations in the normal appearing white matter. ${ }^{27,105}$ Many studies found wide-spread changes in DTI parameters in MS. ${ }^{106-111}$ Here, we found extensive alteration of DTI parameters in the white matter in MS patients compared to HCs. The reason of this broad difference could be the high number of participants in the study, which may lead to the detection of smaller differences. Furthermore, the higher number of MS patients may also contribute because their inclusion leads to an increased percent of focal demyelinating lesions in the skeleton, which have reduced FA and increased MD. ${ }^{111}$ However, only a small proportion of the skeleton contains lesions $(7.5 \%$ of the total volume of the skeleton in a subpopulation of $40 \mathrm{MS}$ patients from the current sample of whom manually segmented lesion mask were available). The mean difference in the significant voxels of FA and AD decreased with the NDED, that is, the two groups mean values depended on NDED in different way. In the lowest NDED, the group difference was the highest. One possible explanation could be the underlying pathology in MS. The white matter FA value is decreased throughout the brain, which is in turn, more prone to overestimation. Moreover, voxels included in the skeleton in the TBSS approach are more ambiguous. The significant change of group differences between the directions is still very low. Regarding the two skeleton sizes, the group differences of the parameters followed a similar trend. In addition, the number of significant voxels and the skeleton size was different between different schemes and decreased with the NDED. The overestimation of skeleton size could be the consequence of the overestimation of FA within the small FA areas. In addition, the elevated number of significant voxels of FA simply resulted 
from the higher number of voxels. This effect could be reversed because correcting for skeleton size, the number of significant voxels of FA was equal as well. Conversely, the number of significant voxels of $\mathrm{MD}$ and $\mathrm{AD}$ was smaller at 15 directions compared to higher directions.

As we worked with fixed encoding directions and their subsets, the distribution of directions could differ from the optimal distribution. According to Zhan et al., this difference was in the range of 5-6\% in terms of distribution energy in 10 and 15 directions ${ }^{39}$ and as Zhan et al. acknowledged this "suboptimal sampling may play a minor role in the SNR gains". We repeated their calculation and the ratio of distribution energy in our study was less than $1 \%$ which is a negligible difference. The difference between the two studies stems from the different number of encoding direction used in the original dataset, that is 94 compared to 60 in our case.

One of the limitations of our study is that the results only applicable to differences between MS and healthy patients, other diseases might show different pattern of TBSS' change as the function of NDED. Other parameters which has an effect on signal-to-noise should also be considered such as voxel size and field strength. 


\section{Conclusion}

In conclusion, eye movements are substantially affected in MS patients, which reflected in several behavior parameters. Moreover, subclinical involvement of the oculomotor system is detected in several cases and highlights the possible use of eye tracker systems to adequately quantify disease burden. The global and focal gray matter alterations are associated with brain areas important in cognitive functions, such as attention. To correctly test white matter integrity, the optimization of diffusion tensor imaging was evaluated considering the number of diffusion encoding directions. Bias caused by directions is more pronounced for areas with small FA values and seems to be constant above 30 directions. Moreover, NDED has a slight effect on TBSS, which makes it reasonable to cautiously compare results from different TBSS studies with different NDED. Regarding all the derived DTI parameters 30 directions might be enough to compare healthy and multiple sclerosis patients with TBSS analysis. Importantly, our results indicate that higher FA threshold of the skeleton should be used with lower NDED to avoid false positive results. White matter integrity could be tested with the optimized sequence and its association with oculomotor deficits as well. As eye tracker systems are promising tools to monitor disease progression the follow-up of our cohort could aid our understanding of eye movements as a predicting biomarker. 


\section{Acknowledgements}

I have to give my greatest gratitude to my supervisor, Zsigmond Tamás Kincses for his help and guidance.

I would like to thank my colleagues and friends, Nikoletta Szabó, Tamás Spisák, András Király, Dániel Veréb, Krisztián Kocsis, Bence Bozsik, Péter Faragó, Eszter Tóth, Péter Bodnár, Melinda Katona for their help and the professional discussions. I wish to express my special gratitude to Brigitta Lóczi and János Hérák Benjámin, graduate students who helped me in the measurements. Special thanks to János Laczi and Zoltán Tóth for their help and sharing their practical knowledge in MRI.

I would like to thank Professor László Vécsei and Professor Péter Klivényi, previous and current Head of the Department of Neurology, for giving me the opportunity to work in the department.

I would like to thank every fellow worker of the Department of Neurology for their help and support.

I would also thank to my family, my wife and my friends for their patience and support.

Last but not least, I would like to thank all the subjects who participated in the studies and contributed to this work. 


\section{References}

1. Dobson R, Giovannoni G. Multiple sclerosis - a review. Eur J Neurol. 2019;26(1):2740. doi:10.1111/ene.13819

2. Kurtzke JF. Rating neurologic impairment in multiple sclerosis: An expanded disability status scale (EDSS). Neurology. 1983;33(11):1444-1452. doi:10.1212/wnl.33.11.1444

3. Serra A, Chisari CG, Matta M. Eye Movement Abnormalities in Multiple Sclerosis: Pathogenesis, Modeling, and Treatment. Front Neurol. 2018;9(FEB):31. doi:10.3389/fneur.2018.00031

4. De Stefano N, Giorgio A, Tintoré M, et al. Radiologically isolated syndrome or subclinical multiple sclerosis: MAGNIMS consensus recommendations. Mult Scler. 2018;24(2):214-221. doi:10.1177/1352458517717808

5. Goodin DS. Glucocorticoid treatment of multiple sclerosis. In: Handbook of Clinical Neurology. Vol 122. Elsevier B.V.; 2014:455-464. doi:10.1016/B978-0-444-52001$2.00020-0$

6. Pearce JMS. Historical descriptions of multiple sclerosis: The stories of Augustus d'Este and The Journal of a Disappointed Man. Eur Neurol. 2005;54(1):49-53. doi: $10.1159 / 000087387$

7. Lassmann H. Pathology and disease mechanisms in different stages of multiple sclerosis. J Neurol Sci. 2013;333(1-2):1-4. doi:10.1016/j.jns.2013.05.010

8. Pérez-Miralles F, Sastre-Garriga J, Tintoré M, et al. Clinical impact of early brain atrophy in clinically isolated syndromes. Mult Scler J. 2013;19(14):1878-1886. doi:10.1177/1352458513488231

9. Atkinson AJ, Colburn WA, DeGruttola VG, et al. Biomarkers and surrogate endpoints: Preferred definitions and conceptual framework. Clin Pharmacol Ther. 2001;69(3):8995. doi:10.1067/mcp.2001.113989

10. Paul A, Comabella M, Gandhi R. Biomarkers in multiple sclerosis. Cold Spring Harb Perspect Med. 2019;9(3). doi:10.1101/cshperspect.a029058

11. Geurts JJG, Calabrese M, Fisher E, Rudick RA. Measurement and clinical effect of grey matter pathology in multiple sclerosis. Lancet Neurol. 2012;11(12):1082-1092. doi:10.1016/S1474-4422(12)70230-2

12. De Stefano N, Giorgio A, Battaglini M, et al. Assessing brain atrophy rates in a large population of untreated multiple sclerosis subtypes. Neurology. 2010;74(23):1868- 
1876. doi:10.1212/WNL.0b013e3181e24136

13. Filippi M. Enhanced magnetic resonance imaging in multiple sclerosis. Mult Scler. 2000;6(5):320-326. doi:10.1177/135245850000600505

14. Cotton F, Weiner HL, Jolesz FA, Guttmann CRG. MRI contrast uptake in new lesions in relapsing-remitting MS followed at weekly intervals. Neurology. 2003;60(4):640646. doi:10.1212/01.WNL.0000046587.83503.1E

15. Kappos L, Moeri D, Radue EW, et al. Predictive value of gadolinium-enhanced magnetic resonance imaging for relapse rate and changes in disability or impairment in multiple sclerosis: A meta-analysis. Lancet. 1999;353(9157):964-969. doi:10.1016/S0140-6736(98)03053-0

16. Zivadinov R, Bakshi R. Role of MRI in multiple sclerosis I: Inflammation and lesions. Front Biosci. 2004;9:665-683. doi:10.2741/1251

17. Bakshi R, Ariyaratana S, Benedict RHB, Jacobs L. Fluid-attenuated inversion recovery magnetic resonance imaging detects cortical and juxtacortical multiple sclerosis lesions. Arch Neurol. 2001;58(5):742-748. doi:10.1001/archneur.58.5.742

18. Filippi M, Preziosa P, Banwell BL, et al. Assessment of lesions on magnetic resonance imaging in multiple sclerosis: practical guidelines. Brain. 2019;142(7):1858-1875. doi:10.1093/brain/awz144

19. Zivadinov R, Leist TP. Clinical-Magnetic Resonance Imaging Correlations in Multiple Sclerosis. J Neuroimaging. 2005;15(4 SUPPL.):10S-21S. doi:10.1177/1051228405283291

20. Bagnato F, Jeffries N, Richert ND, et al. Evolution of T1 black holes in patients with multiple sclerosis imaged monthly for 4 years. Brain. 2003;126(Pt 8):1782-1789. doi:10.1093/brain/awg182

21. Van Walderveen MAA, Kamphorst W, Scheltens P, et al. Histopathologic correlate of hypointense lesions on T1-weighted spin- echo MRI in multiple sclerosis. Neurology. 1998;50(5):1282-1288. doi:10.1212/WNL.50.5.1282

22. Minneboo A, Uitdehaag BMJ, Ader HJ, Barkhof F, Polman CH, Castelijns JA. Patterns of enhancing lesion evolution in multiple sclerosis are uniform within patients. Neurology. 2005;65(1):56-61. doi:10.1212/01.wnl.0000167538.24338.bb

23. Sailer M, Losseff NA, Wang L, Gawne-Cain ML, Thompson AJ, Miller DH. T1 lesion load and cerebral atrophy as a marker for clinical progression in patients with multiple 
sclerosis. A prospective 18 months follow-up study. Eur J Neurol. 2001;8(1):37-42. doi:10.1046/j.1468-1331.2001.00147.x

24. Zivadinov R, Bakshi R. Central nervous system atrophy and clinical status in multiple sclerosis. J Neuroimaging. 2004;14(SUPPL. 3):27S-35S.

doi:10.1177/1051228404266266

25. Bakshi R, Dandamudi VSR, Neema M, De C, Bermel RA. Measurement of brain and spinal cord atrophy by magnetic resonance imaging as a tool to monitor multiple sclerosis. J Neuroimaging. 2005;15(4 SUPPL.):30S-45S. doi:10.1177/1051228405283901

26. Grothe M, Lotze M, Langner S, Dressel A. The role of global and regional gray matter volume decrease in multiple sclerosis. J Neurol. 2016;263(6):1137-1145. doi:10.1007/s00415-016-8114-3

27. Werring DJ, Clark CA, Barker GJ, Thompson AJ, Miller DH. Diffusion tensor imaging of lesions and normal-appearing white matter in multiple sclerosis. Neurology. 1999;52(8):1626-1632. doi:10.1212/wnl.52.8.1626

28. Filippi M, Campi A, Dousset V, et al. A magnetization transfer imaging study of normal-appearing white matter in multiple sclerosis. Neurology. 1995;45(3):478-482. doi:10.1212/WNL.45.3.478

29. Harlow DE, Honce JM, Miravalle AA. Remyelination therapy in multiple sclerosis. Front Neurol. 2015;6(DEC). doi:10.3389/fneur.2015.00257

30. Brown R. XXVII. A brief account of microscopical observations made in the months of June, July and August 1827, on the particles contained in the pollen of plants; and on the general existence of active molecules in organic and inorganic bodies . Philos Mag. 1828;4(21):161-173. doi:10.1080/14786442808674769

31. Fick A. V. On liquid diffusion . London, Edinburgh, Dublin Philos Mag J Sci. 1855;10(63):30-39. doi:10.1080/14786445508641925

32. Einstein A, Cowper AD. INVESTIGATIONS O N THE THEORY .OF,THE BROWNIAN MOVEMENT R. F ü R T H TRANSLATED BY.

33. Stejskal EO, Tanner JE. Spin diffusion measurements: Spin echoes in the presence of a time-dependent field gradient. J Chem Phys. 1965;42(1):288-292. doi:10.1063/1.1695690

34. Basser PJ, Mattiello J, LeBihan D. MR diffusion tensor spectroscopy and imaging. 
Biophys J. 1994;66(1):259-267. doi:10.1016/S0006-3495(94)80775-1

35. Guo AC, MacFall JR, Provenzale JM. Multiple sclerosis: Diffusion tensor MR imaging for evaluation of normal-appearing white matter. Radiology. 2002;222(3):729-736. doi:10.1148/radiol.2223010311

36. Szabó N, Kincses ZT, Párdutz Á, et al. White matter microstructural alterations in migraine: A diffusion-weighted MRI study. Pain. 2012;153(3):651-656. doi:10.1016/j.pain.2011.11.029

37. Soares JM, Marques P, Alves V, Sousa N. A hitchhiker's guide to diffusion tensor imaging. Front Neurosci. 2013;7:31. doi:10.3389/fnins.2013.00031

38. Jones DK, Horsfield MA, Simmons A. Optimal strategies for measuring diffusion in anisotropic systems by magnetic resonance imaging. Magn Reson Med. 1999;42(3):515-525. doi:10.1002/(SICI)1522-2594(199909)42:3<515::AIDMRM14>3.0.CO;2-Q

39. Zhan L, Leow AD, Jahanshad N, et al. How does angular resolution affect diffusion imaging measures? Neuroimage. 2010;49(2):1357-1371. doi:10.1016/j.neuroimage.2009.09.057

40. Hasan KM, Parker DL, Alexander AL. Comparison of gradient encoding schemes for diffusion-tensor MRI. J Magn Reson Imaging. 2001;13(5):769-780. doi:10.1002/jmri.1107

41. Jones DK. The effect of gradient sampling schemes on measures derived from diffusion tensor MRI: A monte carlo study. Magn Reson Med. 2004;51(4):807-815. doi:10.1002/mrm.20033

42. Landman BA, Farrell JAD, Jones CK, Smith SA, Prince JL, Mori S. Effects of diffusion weighting schemes on the reproducibility of DTI-derived fractional anisotropy, mean diffusivity, and principal eigenvector measurements at $1.5 \mathrm{~T}$. Neuroimage. 2007;36(4):1123-1138. doi:10.1016/j.neuroimage.2007.02.056

43. Ni H, Kavcic V, Zhu T, Ekholm S, Zhong J. Effects of number of diffusion gradient directions on derived diffusion tensor imaging indices in human brain. Am J Neuroradiol. 2006;27(8):1776-1781.

44. Papadakis NG, Murrills CD, Hall LD, Huang CLH, Adrian Carpenter T. Minimal gradient encoding for robust estimation of diffusion anisotropy. Magn Reson Imaging. 2000;18(6):671-679. doi:10.1016/S0730-725X(00)00151-X 
45. Batchelor PG, Atkinson D, Hill DLG, Calamante F, Connelly A. Anisotropic noise propagation in diffusion tensor MRI sampling schemes. Magn Reson Med. 2003;49(6):1143-1151. doi:10.1002/mrm.10491

46. Skare S, Hedehus M, Moseley ME, Li TQ. Condition number as a measure of noise performance of diffusion tensor data acquisition schemes with MRI. J Magn Reson. 2000;147(2):340-352. doi:10.1006/jmre.2000.2209

47. Smith SM, Jenkinson M, Johansen-Berg H, et al. Tract-based spatial statistics: Voxelwise analysis of multi-subject diffusion data. Neuroimage. 2006;31(4):14871505. doi:10.1016/j.neuroimage.2006.02.024

48. Mastaglia FL, Black JL, Collins DW. Quantitative studies of saccadic and pursuit eye movements in multiple sclerosis. Brain. 1979;102(4):817-834. doi:10.1093/brain/102.4.817

49. Servillo G, Renard D, Taieb G, et al. Bedside tested ocular motor disorders in multiple sclerosis patients. Mult Scler Int. 2014;2014:732329. doi:10.1155/2014/732329

50. Derwenskus J, Rucker JC, Serra A, et al. Abnormal eye movements predict disability in MS: two-year follow-up. Ann N Y Acad Sci. 2005;1039:521-523. doi:10.1196/annals.1325.058

51. Corbetta M, Kincade JM, Ollinger JM, McAvoy MP, Shulman GL. Voluntary orienting is dissociated from target detection in human posterior parietal cortex. Nat Neurosci. 2000;3(3):292-297. doi:10.1038/73009

52. Pierrot-Deseilligny C, Müri RM, Ploner CJ, Gaymard B, Rivaud-Péchoux S. Cortical control of ocular saccades in humans: a model for motricity. Prog Brain Res. 2003;142:3-17. doi:10.1016/S0079-6123(03)42003-7

53. Cerkevich CM, Lyon DC, Balaram P, Kaas JH. Distribution of cortical neurons projecting to the superior colliculus in macaque monkeys. Eye Brain. 2014;2014(6):121-137. doi:10.2147/EB.S53613

54. Rushworth MFS, Behrens TEJ, Johansen-Berg H. Connection patterns distinguish 3 regions of human parietal cortex. Cereb Cortex. 2006;16(10):1418-1430. doi:10.1093/cercor/bhj079

55. Paré M, Wurtz RH. Monkey posterior parietal cortex neurons antidromically activated from superior colliculus. J Neurophysiol. 1997;78(6):3493-3497. doi:10.1152/jn.1997.78.6.3493 
56. Frohman EM, Frohman TC, O’Suilleabhain P, et al. Quantitative oculographic characterisation of internuclear ophthalmoparesis in multiple sclerosis: the versional dysconjugacy index Z score. J Neurol Neurosurg Psychiatry. 2002;73(1):51-55. doi:10.1136/jnnp.73.1.51

57. Agarwal M, Ulmer JL, Chandra T, Klein AP, Mark LP, Mohan S. Imaging correlates of neural control of ocular movements. Eur Radiol. 2016;26(7):2193-2205. doi:10.1007/s00330-015-4004-9

58. Kincses ZT, Tóth E, Fricska-Nagy Z, et al. Az MRI helye a sclerosis multiplex kezelés hatékonyságának megítélésében II.: mérési protokollok. Ideggyogy Sz. 2018;71(34):81-88. doi:10.18071/isz.71.0081

59. Kincses ZT, Ropele S, Jenkinson M, et al. Lesion probability mapping to explain clinical deficits and cognitive performance in multiple sclerosis. Mult Scler. 2011;17(6):681-689. doi:10.1177/1352458510391342

60. Jenkinson M, Smith S. A global optimisation method for robust affine registration of brain images. Med Image Anal. 2001;5(2):143-156. doi:10.1016/S13618415(01)00036-6

61. Andersson JLR, Jenkinson M, Smith SM. Non-linear optimisation. FMRIB technical report TR07JA1. Pr. 2007;(June):16. doi:10.1109/EMOBILITY.2010.5668100

62. Ashburner J, Friston KJ. Voxel-based morphometry - The methods. Neuroimage. 2000;11(6 I):805-821. doi:10.1006/nimg.2000.0582

63. Good CD, Johnsrude IS, Ashburner J, Henson RNA, Friston KJ, Frackowiak RSJ. A Voxel-Based Morphometric Study of Ageing in 465 Normal Adult Human Brains. 2001. doi:10.1006/nimg.2001.0786

64. Smith SM, Jenkinson M, Woolrich MW, et al. Advances in functional and structural MR image analysis and implementation as FSL. In: NeuroImage. Vol 23. ; 2004. doi:10.1016/j.neuroimage.2004.07.051

65. Smith SM. Fast robust automated brain extraction. Hum Brain Mapp. 2002;17(3):143155. doi: $10.1002 / \mathrm{hbm} .10062$

66. Zhang Y, Brady M, Smith S. Segmentation of brain MR images through a hidden Markov random field model and the expectation-maximization algorithm. IEEE Trans Med Imaging. 2001;20(1):45-57. doi:10.1109/42.906424

67. R Core Team. R: A Language and Environment for Statistical Computing. 2018. 
https://www.r-project.org/.

68. Bates D, Machler M, Bolker B, Walker S. Fitting linear mixed-effects models using lme4. J Stat Software, Artic. 2015;67(1):1-48. doi:10.18637/jss.v067.i01

69. Fox J, Weisberg S. An R Companion to Applied Regression. 2nd editio. Thousand Oaks CA: Sage; 2014. doi:10.1177/0049124105277200

70. Jenkinson M, Bannister P, Brady M, Smith S. Improved optimization for the robust and accurate linear registration and motion correction of brain images. Neuroimage. 2002;17(2):825-841. doi:10.1016/S1053-8119(02)91132-8

71. Searle SR, Speed FM, Milliken GA. Population marginal means in the linear model : An alternative to least squares means. Am Stat. 1980;34(4):216-221.

72. Clough M, Millist L, Lizak N, et al. Ocular motor measures of cognitive dysfunction in multiple sclerosis I: inhibitory control. J Neurol. 2015;262(5):1130-1137. doi:10.1007/s00415-015-7645-3

73. Clough M, Mitchell L, Millist L, et al. Ocular motor measures of cognitive dysfunction in multiple sclerosis II: working memory. J Neurol. 2015;262(5):1138-1147. doi:10.1007/s00415-015-7644-4

74. Fielding J, Kilpatrick T, Millist L, Clough M, White O. Longitudinal assessment of antisaccades in patients with multiple sclerosis. PLoS One. 2012;7(2):e30475. doi:10.1371/journal.pone.0030475

75. Fielding J, Kilpatrick T, Millist L, White O. Multiple sclerosis: Cognition and saccadic eye movements. J Neurol Sci. 2009;277(1-2):32-36. doi:10.1016/j.jns.2008.10.001

76. Fielding J, Kilpatrick T, Millist L, White O. Antisaccade performance in patients with multiple sclerosis. Cortex. 2009;45(7):900-903. doi:10.1016/j.cortex.2009.02.016

77. Fielding J, Kilpatrick T, Millist L, White O. Control of visually guided saccades in multiple sclerosis: Disruption to higher-order processes. Neuropsychologia. 2009;47(7):1647-1653. doi:10.1016/j.neuropsychologia.2009.01.040

78. Finke C, Pech LM, Sömmer C, et al. Dynamics of saccade parameters in multiple sclerosis patients with fatigue. J Neurol. 2012;259(12):2656-2663. doi:10.1007/s00415-012-6565-8

79. Kolbe SC, Kilpatrick TJ, Mitchell PJ, White O, Egan GF, Fielding J. Inhibitory saccadic dysfunction is associated with cerebellar injury in multiple sclerosis. Hum Brain Mapp. 2014;35(5):2310-2319. doi:10.1002/hbm.22329 
80. Chen YF, Chen T, Tsai TT. Analysis of volition latency on antisaccadic eye movements. Med Eng Phys. 1999;21(8):555-562. doi:10.1016/S1350-4533(99)00082$\mathrm{X}$

81. Munoz DP, Everling S. Look away: The anti-saccade task and the voluntary control of eye movement. Nat Rev Neurosci. 2004;5(3):218-228. doi:10.1038/nrn1345

82. Giorgio A, Stromillo ML, Bartolozzi ML, et al. Relevance of hypointense brain MRI lesions for long-term worsening of clinical disability in relapsing multiple sclerosis. Mult Scler. 2014;20(2):214-219. doi:10.1177/1352458513494490

83. Kincses ZT, Tóth E, Bankó N, et al. Grey matter atrophy in patients suffering from multiple sclerosis. Ideggyogy $S z$. 2014;67(9-10):293-300. http://www.ncbi.nlm.nih.gov/pubmed/25518257. Accessed March 15, 2020.

84. Paolillo A, Pozzilli C, Gasperini C, et al. Brain atrophy in relapsing-remitting multiple sclerosis: Relationship with "black holes", disease duration and clinical disability. $J$ Neurol Sci. 2000;174(2):85-91. doi:10.1016/S0022-510X(00)00259-8

85. Wegner C, Esiri MM, Chance SA, Palace J, Matthews PM. Neocortical neuronal, synaptic, and glial loss in multiple sclerosis. Neurology. 2006;67(6):960-967. doi:10.1212/01.wnl.0000237551.26858.39

86. Rocca MA, Comi G, Filippi M. The Role of T1-Weighted Derived Measures of Neurodegeneration for Assessing Disability Progression in Multiple Sclerosis. Front Neurol. 2017;8(SEP):433. doi:10.3389/fneur.2017.00433

87. Di Stasi LL, Catena A, Cañas JJ, Macknik SL, Martinez-Conde S. Saccadic velocity as an arousal index in naturalistic tasks. Neurosci Biobehav Rev. 2013;37(5):968-975. doi:10.1016/j.neubiorev.2013.03.011

88. Di Stasi LL, Renner R, Staehr P, et al. Saccadic peak velocity sensitivity to variations in mental workload. Aviat Sp Environ Med. 2010;81(4):413-417. doi:10.3357/ASEM.2579.2010

89. Dumoulin SO. A New Anatomical Landmark for Reliable Identification of Human Area V5/MT: a Quantitative Analysis of Sulcal Patterning. Cereb Cortex. 2000;10(5):454-463. doi:10.1093/cercor/10.5.454

90. Corbetta M, Shulman GL. Control of goal-directed and stimulus-driven attention in the brain. Nat Rev Neurosci. 2002;3(3):201-215. doi:10.1038/nrn755

91. Mai JK, Paxinos G. The Human Nervous System. Elsevier Inc.; 2012. 
doi:10.1016/C2009-0-02721-4

92. Karnath HO, Rorden C. The anatomy of spatial neglect. Neuropsychologia. 2012;50(6):1010-1017. doi:10.1016/j.neuropsychologia.2011.06.027

93. Gilad R, Sadeh M, Boaz M, Lampl Y. Visual spatial neglect in multiple sclerosis. Cortex. 2006;42(8):1138-1142. doi:10.1016/S0010-9452(08)70226-0

94. Pierrot-Deseilligny C, Milea D, Müri RM. Eye movement control by the cerebral cortex. Curr Opin Neurol. 2004;17(1):17-25. doi:10.1097/00019052-200402000-00005

95. Everling S, Spantekow A, Krappmann P, Flohr H. Event-related potentials associated with correct and incorrect responses in a cued antisaccade task. Exp Brain Res. 1998;118(1):27-34. doi:10.1007/s002210050252

96. Gottlieb J, Goldberg ME. Activity of neurons in the lateral intraparietal area of the monkey during an antisaccade task. Nat Neurosci. 1999;2(10):906-912. doi:10.1038/13209

97. Tóth E, Faragó P, Király A, et al. The contribution of various MRI parameters to clinical and cognitive disability in multiple sclerosis. Front Neurol. 2019;9:1172. doi:10.3389/fneur.2018.01172

98. Basser PJ, Mattiello J, Lebihan D. Estimation of the effective self-diffusion tensor from the NMR spin echo. J Magn Reson Ser B. 1994;103(3):247-254. doi:10.1006/jmrb.1994.1037

99. Chen Y, Tymofiyeva O, Hess CP, Xu D. Effects of rejecting diffusion directions on tensor-derived parameters. Neuroimage. 2015;109:160-170. doi:10.1016/j.neuroimage.2015.01.010

100. Farrell JAD, Landman BA, Jones CK, et al. Effects of signal-to-noise ratio on the accuracy and reproducibility of diffusion tensor imaging-derived fractional anisotropy, mean diffusivity, and principal eigenvector measurements at 1.5T. J Magn Reson Imaging. 2007;26(3):756-767. doi:10.1002/jmri.21053

101. Pfefferbaum A, Sullivan E V. Increased brain white matter diffusivity in normal adult aging: Relationship to anisotropy and partial voluming. Magn Reson Med. 2003;49(5):953-961. doi:10.1002/mrm.10452

102. Calabrese M, Filippi M, Gallo P. Cortical lesions in multiple sclerosis. Nat Rev Neurol. 2010;6(8):438-444. doi:10.1038/nrneurol.2010.93

103. Popescu V, Agosta F, Hulst HE, et al. Brain atrophy and lesion load predict long term 
disability in multiple sclerosis. J Neurol Neurosurg Psychiatry. 2013;84(10):10821091. doi:10.1136/jnnp-2012-304094

104. Tóth E, Szabó N, Csete G, et al. Gray matter atrophy is primarily related to demyelination of lesions in multiple sclerosis: A diffusion tensor imaging MRI study. Front Neuroanat. 2017;11:23. doi:10.3389/FNANA.2017.00023

105. Laganà M, Rovaris M, Ceccarelli A, Venturelli C, Marini S, Baselli G. DTI parameter optimisation for acquisition at 1.5T: SNR analysis and clinical application. Comput Intell Neurosci. 2010;2010:23. doi:10.1155/2010/254032

106. Huang J, Liu Y, Zhao T, et al. White matter microstructural alterations in clinically isolated syndrome and multiple sclerosis. J Clin Neurosci. 2018;53:27-33. doi:10.1016/j.jocn.2018.01.007

107. Preziosa P, Rocca MA, Mesaros S, et al. Intrinsic damage to the major white matter tracts in patients with different clinical phenotypes of multiple sclerosis: A voxelwise diffusion-tensor MR study. Radiology. 2011;260(2):541-550. doi:10.1148/radiol.11110315

108. Roosendaal SD, Geurts JJG, Vrenken H, et al. Regional DTI differences in multiple sclerosis patients. Neuroimage. 2009;44(4):1397-1403. doi:10.1016/j.neuroimage.2008.10.026

109. Bodini B, Khaleeli Z, Cercignani M, Miller DH, Thompson AJ, Ciccarelli O. Exploring the relationship between white matter and gray matter damage in early primary progressive multiple sclerosis: an in vivo study with TBSS and VBM. Hum Brain Mapp. 2009;30(9):2852-2861. doi:10.1002/hbm.20713

110. Schoonheim MM, Vigeveno RM, Rueda Lopes FC, et al. Sex-specific extent and severity of white matter damage in multiple sclerosis: implications for cognitive decline. Hum Brain Mapp. 2014;35(5):2348-2358. doi:10.1002/hbm.22332

111. Rovaris M, Gass A, Bammer R, et al. Diffusion MRI in multiple sclerosis. Neurology. 2005;65(10):1526-1532. doi:10.1212/01.wnl.0000184471.83948.e0 
1 


\section{OPEN ACCESS}

Edited by:

Elena H. Martínez-Lapiscina, European Medicines Agency, United Kingdom

Reviewed by: Larry Allen Abel,

The University of Melbourne, Australia

Christian Cordano,

University of California, San Francisco,

United States

*Correspondence:

Zsigmond Tamás Kincses kincses.zsigmond.tamas@ med.u-szeged.hu

Specialty section: This article was submitted to Neuro-Ophthalmology, a section of the journal Frontiers in Neurology

Received: 22 December 2018 Accepted: 20 May 2019

Published: 04 June 2019

Citation:

Kincses B, Hérák BJ, Szabó N, Bozsik $B$, Faragó $P$, Király $A$, Veréb $D$, Tóth E, Kocsis K, Bencsik K, Vécsei L and Kincses ZT (2019) Gray Matter Atrophy to Explain Subclinical Oculomotor Deficit in Multiple Sclerosis. Front. Neurol. 10:589. doi: 10.3389/fneur.2019.00589

\section{Gray Matter Atrophy to Explain Subclinical Oculomotor Deficit in Multiple Sclerosis}

\author{
Bálint Kincses ${ }^{1}$, Benjámin J. Hérák ${ }^{1}$, Nikoletta Szabó ${ }^{1}$, Bence Bozsik ${ }^{1}$, Péter Faragó ${ }^{1}$, \\ András Király ${ }^{1}$, Dániel Veréb ${ }^{1}$, Eszter Tóth ${ }^{1}$, Krisztián Kocsis ${ }^{1}$, Krisztina Bencsik ${ }^{1}$, \\ László Vécsei ${ }^{1,2}$ and Zsigmond Tamás Kincses ${ }^{1,3 *}$

\footnotetext{
${ }^{1}$ Department of Neurology, Albert Szent-Györgyi Clinical Center, University of Szeged, Szeged, Hungary, ${ }^{2}$ MTA-SZTE Neuroscience Research Group, Szeged, Hungary, ${ }^{3}$ Department of Radiology, Albert Szent-Györgyi Clinical Center, University of Szeged, Szeged, Hungary
}

Eye movement deficits are frequently noted in multiple sclerosis during bedside clinical examination, but subtle dysfunction may remain undetected and might only be identified with advanced approaches. While classical neurology provides insight into the complex functional anatomy of oculomotor functions, little is known about the structural background of this dysfunction in MS. Thirty four clinically stable, treated relapsing-remitting MS patients with mild disability and 34 healthy controls were included in our study. Group difference and correlation with clinical parameters were analyzed in case of the latency, peak-velocity, gain, dysconjugacy index, and performance during a saccade and anti-saccade task. High-resolution T1 weighted, T2 FLAIR, and double inversion recovery images were acquired on $3 \mathrm{~T}$ to evaluate the correlation between behavioral and MRI parameters, such as T2 lesion and T1 black-hole burden, global brain, gray, and white matter atrophy. VBM style analysis was used to identify the focal gray matter atrophy responsible for oculomotor dysfunction. Significantly increased latency in the prosaccade task and significantly worse performance in the anti-saccade task were found in MS patients. The detailed examination of conjugated eye movements revealed five subclinical internuclear ophthalmoparesis cases. The peak velocity and latency of the anti-saccade movement correlated with the number of black holes, but none of the eye movement parameters were associated with the T2 lesion burden or location. Global gray matter volume correlated with saccade and anti-saccade latency, whereas white matter and total brain volume did not. Local gray matter atrophy in the left inferio-parietal lobule and temporo-occipital junction correlated with anti-saccade peak velocity. Our results show that neurodegeneration-like features of the MRI (black-hole, gray matter atrophy) are the best predictors of eye movement deficit in MS. Concurring with the clinico-radiological paradox, T2 lesion burden cannot explain the behavioral results. Importantly, anti-saccade peak velocity correlates with gray matter atrophy in the left parietal regions, which are frequently implicated in attention tasks.

\footnotetext{
Keywords: multiple sclerosis, prosaccade, anti-saccade, atrophy, black hole
} 


\section{INTRODUCTION}

Multiple sclerosis (MS) is a devastating disease that mostly affects young adults. Among many other symptoms, oculomotor deficit is common in MS, reported to occur in $57-70 \%$ of all patients $(1,2)$. Its significance lies in the observation that the presence of eye movement abnormality is associated with greater disability and greater disability progression (3). Bedside oculo-motor examination by an experienced specialist could reveal major oculomotor deficits, but subtle alterations might remain undetected. Eye tracker devices are suitable for objective and quantitative measurements of eye movements and are more sensitive in detecting subclinical abnormalities (1).

The aim of the eye movements is to keep the object of interest on the fovea. These voluntary and reflexive movements are the rapid jerky saccades, the smooth pursuit and vergence movements. These intricate ocular movements are accomplished by six extraocular muscles, the movement of which is coordinated by a complex network of cortical and subcortical neuronal elements.

The main purpose of the rapid voluntary conjugate eye movements known as saccades, is to bring the new object of interest onto the foveae. The cranial nerve nuclei of the oculomotor muscles could be found in the brainstem. In addition, other elements of the premotor circuits of saccades are in the brainstem such as the paramedian pontine reticular formation, nucleus of the medial longitudinal fasciculus and nucleus raphe interpositius. All of these regions receive afferents from the superior colliculus. Moreover, the behaviorally important stimuli are processed in various cortical networks and together with fronto-parietal attention networks have crucial role in guiding eye movements during saccades (4). Eventually the cortical signals for eye movements are generated in the frontal eye filed (FEF) in close interaction with other centers such as supplementary and pre-supplementary eye fields (5), the dorsolateral prefrontal cortex and parietal cortex. From the cortical centers the information is conveyed via the superior colliculus to the nuclei of the oculomotor nerves directly and indirectly as well (6-8). Over the course of information flow various subcortical, brainstem and cerebellar centers are modulating the process.

Damage to certain parts of this network causes clinically abnormal eye movement $(9,10)$ some of those easily detectable by bedside examination (11). However, the structural background of subtle eye movement deficits in MS is not well-understood. Damage of the perceptual systems, the cognitive networks such as attention and the eye movement centers cause various alterations of eye movements.

The aim of our study was to investigate the subclinical oculomotor deficit of MS patients in prosaccade and antisaccade tasks. The higher order structural background of such abnormal eye movements was investigated by correlating the behavioral measures with lesion location and gray matter atrophy.

\section{MATERIALS AND METHODS}

\section{Subjects}

Thirty nine relapsing remitting MS patients were enrolled in our study. Inclusion criteria for patients were: relapsing remitting MS on disease modifying treatment, EDSS score $<6$, no relapse in the preceding 3 months, no other major neurological, psychiatric or ophthalmological disease (for clinical and demographic data see Table 1).

We also recruited 34 healthy controls (HC), who had no major neurological, psychiatric, or ophthalmological disease.

This study was carried out in accordance with the recommendations of the Medical Research Council National Scientific and Ethical Committee (ETT TUKEB) with written informed consent from all subjects. All subjects gave written informed consent in accordance with the Declaration of Helsinki. The protocol was approved by the National Institute of Pharmacy and Nutrition (000002/2016/OTIG).

\section{Visuo-Motor Task}

The subjects completed a prosaccade and an anti-saccade task. The investigation took place in a well-lit room. The subjects sat $60 \mathrm{~cm}$ away from the screen. The visual stimuli and the task paradigm were written using the Tobii MATLAB binding ${ }^{1}$ and the Psychophysics Toolbox Version 3.0.12 ${ }^{2}$, under MatLab 8.3.0.532 (2014a, MathWorks, Inc.). Eye movement recording was carried out with a Tobii TX300 eyetracker. Before the task, a 5 points calibration was carried out. The prosaccade task was the following: A black cross appeared in the center of a gray screen, which disappeared after a random interval of $1.2-2 \mathrm{~s}$ and appeared instantaneously in the left or right side of the screen, 9.2 or $18.4^{\circ}$ From the center. Each condition ( 4 in all: leftfar, left-close, right-far, right-close) was repeated 20 times in a pseudorandom order. Subjects had to move their gaze to the new location of the target instantly and accurately. Halfway during the task, there was a break to prevent subjects from fatigue and/or tearing. In the anti-saccade task, the layout was the same, but the subjects had to move their gaze contralateral to the position of the new target.

The data acquisition started when the target (cross) jumped to the periphery and lasted $1 \mathrm{~s}$. The sampling frequency was $300 \mathrm{~Hz}$. Data from both eyes were recorded simultaneously. Each recorded data point had a time stamp and a validity code. After the data acquisition, the target jumped back to the center of the screen.

\section{Data Processing}

The recorded data was processed offline. Trials in which more than $10 \%$ of the data was missing (validity code higher than 1 as provided by the eye tracker) or more than $100 \mathrm{~ms}$ was missing continuously or more than $80 \%$ was missing in the first $50 \mathrm{~ms}$ were excluded from further analysis. In the rest of the trials, missing values were interpolated with linear interpolation of the neighboring values.

\footnotetext{
${ }^{1}$ http://developer.tobiipro.com/matlab.html

${ }^{2}$ http://psychtoolbox.org/
} 
TABLE 1 | Demographic data of the subjects.

\begin{tabular}{|c|c|c|c|c|c|c|}
\hline Group & $\#$ of subjects & Females & $\begin{array}{c}\text { Age } \\
\text { (years) }\end{array}$ & EDSS & $\begin{array}{l}\text { Disease duration } \\
\text { (months) }\end{array}$ & Treatment regimen \\
\hline MS & 39 & 25 & $39.1( \pm 9.5)$ & $1.4( \pm 1.4)$ & 103( \pm 72.8$)$ & $\begin{array}{c}\text { DF-15\% } \\
\text { Te-28\% } \\
\text { IFNb-13\% } \\
\text { GA-18\% } \\
\text { F-26\% }\end{array}$ \\
\hline $\mathrm{HC}$ & 34 & 23 & $31( \pm 10.9)$ & - & - & - \\
\hline
\end{tabular}

$D F$, dimethyl fumarate; Te, teriflunodamide; IFNb, interferon beta 1a; GA, glatiramer acetate; F, fingolimid.

The preprocessed data were smoothed and differentiated with a $0,1^{\text {st }}$ and $2^{\text {nd }}$ order 11 -points sliding window Savitzky-Golay filter ${ }^{3}$ to calculate the position, velocity and acceleration of the eyes. Saccades were detected automatically: if the velocity of the eye exceeded $50 \%$ in 2 consecutive points it was labeled as a saccade like event. The beginning of the saccade like event was marked where the acceleration of the eye was 0 (or reached its minimum value in $50 \mathrm{~ms}$ before its peak). The end of the saccade like event was marked where its velocity reached zero after the peak. Saccade like motion was accepted as a saccade if its latency occurred between 100 and $600 \mathrm{~ms}$ after stimulus onset, it took at least $12 \mathrm{~ms}$ and a fixation preceded the saccade like event. During fixation the eye had to be close to the initial cross $\left(<1.5^{\circ}\right)$ and its position change over the fixation had to be $<0.6^{\circ}$. For all trials the position-time diagram was checked visually and inadequate trials were excluded. The first two trials in each condition were seen as practice and excluded from further analysis. A condition was accepted if the subject had at least 9 trials (half of the trials in a condition) after exclusion. Saccade latency (the start of the saccade), saccade peak velocity, saccade amplitude and saccade duration were assessed. Saccade gain was calculated from the ratio of the final eye position and the target position. Antisaccade latency, gain, peak velocity were determined similarly in the correctly performed trials. Anti-saccade performance was calculated as the percentage of correctly performed trials to all the adequate trials. Moreover, a dysconjugacy index (DI) was calculated in the saccade task from both eyes as the ratio of the abducting and adducting eye's velocity in the "long" condition. DI was determined in the left and right directions. Patient's $Z$-scores were calculated as indicated in Equation 1.

$$
Z_{D I}=\frac{D I(\mathrm{MS})-\text { mean }(D I(H C))}{S T D(D I(H C))}
$$

\section{Magnetic Resonance Imaging}

Magnetic resonance imaging was performed with a $3 \mathrm{~T}$ GE Discovery 750 w MR Scanner (GE Healthcare, Chalfont St. Giles, UK). The MR images used in the current study were acquired as part of the routine follow up of the patients, the protocol of which is described in details in our recent recommendation (12). The following sequences were used in the current analysis: High resolution $\mathrm{T} 1$ weighted anatomical images $(3 \mathrm{D}$ spoiled

\footnotetext{
${ }^{3}$ https://www.mathworks.com/matlabcentral/fileexchange/30299-savitzky-golaysmooth-differentiation-filters-and-filterapplication
}

gradient echo images with inversion recovery (3D FSPGR IR: echo time [TE]: $2 \mathrm{~ms}$; repetition time [TR]: $5.4 \mathrm{~ms}$; inversion time [TI]: $450 \mathrm{~ms}$; matrix: $256 * 256$; field of view [FOV]: $25.6 \mathrm{~cm} *$ $25.6 \mathrm{~cm}$; flip angle: $12^{\circ}$; slice thickness [sl]: $1 \mathrm{~mm}$; PURE intensity correction), CUBE T2 FLAIR for lesion detection (TE: $135 \mathrm{~ms}$; TR: $6700 \mathrm{~ms}$; TI: $1827 \mathrm{~ms}$; matrix: 256*224; FOV: $25^{*} 22.5 \mathrm{~cm}$, sl: $1.4 \mathrm{~mm}$; fat sat; post processing: ZIP512, ZIP2), CUBE double inversion recovery (DIR) (TE: $90 \mathrm{~ms}$; TR: 7,000 ms; TI: 2,901 ms; blood suppression TI: $546 \mathrm{~ms}$; matrix: 192*192; FOV: $25 \mathrm{~cm} *$ $25 \mathrm{~cm}$; sl: $1.4 \mathrm{~mm}$; fat sat) and spin echo (SE) T1 weighted images were acquired (TE: min full, TR: 500, flip angle: $73^{\circ}$; matrix: 256*224, FOV: $24 \mathrm{~cm} *$ 19.2, sl: 3 mm, NEX: 2).

\section{Image Analysis}

Lesion load was determined in the periventricular, infratentorial, and juxtacortical regions on the FLAIR and DIR images manually. Lesion load in the whole brain as well as in the above-mentioned subregions was correlated with the behavior parameters. The SE T1 images were used for determine black hole burden.

The correlation of lesion location probability and eye movement deficit was evaluated as described by Kincses et al. (13). Binary lesion mask were brought into standard space by registering the FLAIR images to the high resolution T1 weighted images by 6 DOF linear registration (14) and the T1 weighted images to standard MNI152 space by non-linear registration (15). The standard space binary lesion masks were concatenated. A voxelwise GLM analysis was performed, the regressors of the design matrix were the measured eye movement parameters. Non-parametric permutation test, with 5,000 permutations were used for statistical inference with correction for multiple comparisons.

The high resolution T1 weighted images were used for voxelbased morphometry analysis. We employed an "optimized" VBM-style protocol $(16,17)$ using FSL (18). Non-brain parts were removed from all structural images (19) and tissue-type segmentation was carried out by FAST4 (20). The resulting gray matter partial volume images were registered to standard space (MNI152) using linear transformation (14) followed by a nonlinear registration (15). The resulting images were averaged to create a study-specific template, to which the native gray matter images were then non-linearly re-registered. The registered partial volume images were then modulated (to correct for local expansion or contraction) by dividing by the Jacobian of the warp 
field. The modulated segmented images were then smoothed with an isotropic Gaussian kernel with a sigma of $2 \mathrm{~mm}$. Finally, voxelwise GLM was applied and permutation-based non-parametric testing correcting for multiple comparisons across space was used for statistical inference. The design matrix contained the behavior parameters (saccade latency, peak velocity, and gain) in consecutive analyses. The model was adjusted for disease duration and age. Thresholding was carried out by cluster-based thresholding corrected for multiple comparison by using cluster size.

\section{Statistical Analysis}

Statistical analysis was carried out with Rstudio (21). The following packages were used: Ime4 (22) - model building, car (23) - statistical significance. Mean and standard error were calculated for the following parameters: latency, peak velocity and gain from both eyes in the prosaccade and anti-saccade tasks separately. All parameters were evaluated in a mixed model ANOVA, in which the subject was the random effect and the group (HC-MS), the movement type (abduction-adduction), and the distance (far-close) of the target handled as fixed effects. A $p<0.05$ was considered significant. We investigated both eyes separately because an average of the two eyes could be misleading if subclinical internuclear ophthalmoparesis coexists. The results from the left eye were reported unless otherwise stated. To investigate oculomotor decision, latency and peak velocity differences between prosaccade/anti-saccade tasks were calculated and compared between the groups. The name of the new calculated variables were peak velocity difference and latency difference. As peak velocity and amplitude have linear relationship in case of significance the statistic was repeated with the scaled peak velocities. Pearson or Spearman correlation (where the assumptions of the Pearson were not valid) between MRI markers and behavior parameters were calculated in separate analyses. The effect of disease duration and age was tested in partial correlation and the effect of sex is tested via comparing the Fischer $\mathrm{Z}$ transformed correlation coefficients in the two sex separately. Correlation coefficient are reported from simple correlation where the age, disease duration and sex had no effect on the association.

\section{RESULTS}

Clinical examination indicated that 5 patients (13\%) had clinically detectable oculomotor alteration. They were excluded from further quantitative analysis. One further patients was also excluded because of technical issues with the MRI images. The demographical data of subjects are presented in Table 1. All patients were on disease modifying therapy (6 patients take dimethyl-fumarate, 11-teriflonomide, 4- i.m. interferon beta1a, 7-glatiramer acetate, 10-fingolimod, 1-s.c interferon beta1a).

The average total lesion number was $21( \pm 15)$. As expected, most of the lesions occurred in the periventricular region (12.5 \pm 7.6$)$, but significant lesion load was found in the infratentorial and juxtacortical location too (1.5 $\pm 1.6,3.6$ \pm 4.5 , respectively).

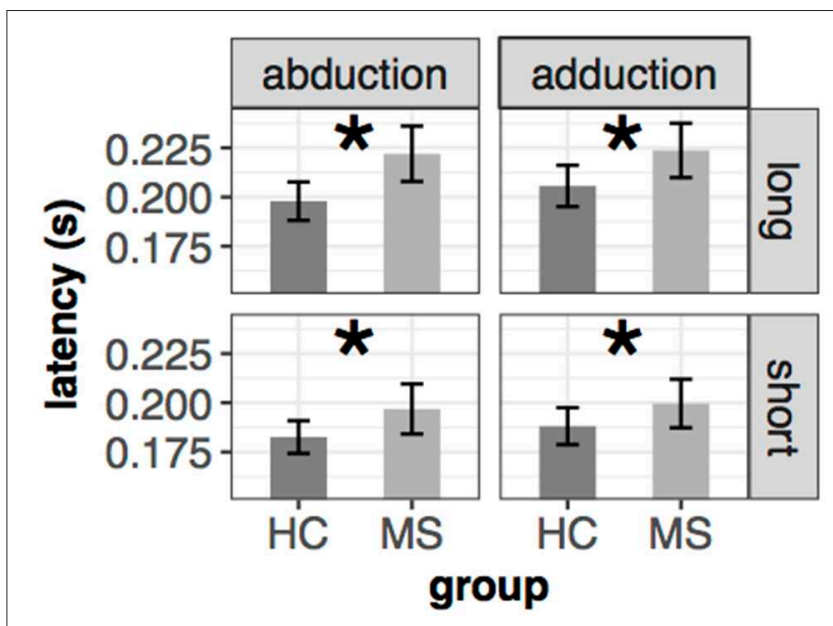

FIGURE 1 | Mean and \pm SEM of latency in different conditions of the left eye in the saccade task. Significant group difference is marked with an asterisk ( $\left(^{\star}\right)$. The mean and standard error from the left-upper part of the figure were the following: 198( \pm 5 )ms vs. 221( \pm 7 )ms (abduction+long), 206( \pm 5$) \mathrm{ms}$ vs. 224( \pm 7$) \mathrm{ms}$ (adduction+long), 183( \pm 4 )ms vs. 197( \pm 6$) \mathrm{ms}$ (abduction+short), $188( \pm 5) \mathrm{ms}$ vs. 200( \pm 6$) \mathrm{ms}$ (adduction+short) for HC and MS, respectively.

\section{Latency}

The latency of the anti-saccades were longer than the latency of saccades (194 ms vs. $303 \mathrm{~ms}, t=-17.3, p<0.0001$ for HCs and $207 \mathrm{~ms}$ vs. $319 \mathrm{~ms}, t=-18.6, p<0.0001$ for MS participants).

Saccade latency was significantly prolonged in MS patients. The results were similar in both eyes [left: $F_{(1,65.966)}=5.36$, $p=0.024$, right: $F_{(1,65.98)}=5.38, p=0.024$ ] [mean $( \pm \mathrm{sd})$ : $194( \pm 24) \mathrm{ms}$ vs. $207( \pm 31) \mathrm{ms}$ for HC and MS participants]. There were no interaction effects between the fixed effects (group, movement type and distance of the target). The results are depicted in Figure 1.

Anti-saccade latency was prolonged in MS patients, however it did not reach a significant level $\left[F_{(1,64.96)}=2.39, p=0.12\right]$ [mean $( \pm \mathrm{sd}): 303( \pm 44)$ ms vs. $319( \pm 44) \mathrm{ms}$ for $\mathrm{HC}$ and MS, respectively].

The latency difference between prosaccade and anti-saccade tasks was also investigated on group level and we found no significant difference. $\left[t_{(64)}=0.29, p=0.77\right]$ [mean $( \pm \mathrm{sd})$ : 107( \pm 36$) \mathrm{ms}$ vs. 112( \pm 34$) \mathrm{ms}$ for HC and MS, respectively].

\section{Peak Velocity}

Prosaccade peak velocity was slightly smaller in the MS group [mean $( \pm \mathrm{sd}): 325( \pm 32)^{\circ} / \mathrm{s}$ vs. $310( \pm 42)^{\circ} / \mathrm{s}$ for $\mathrm{HC}$ and MS, respectively] but there was no significant difference between the two groups $\left[F_{(1,65.96)}=1.4, p=0.28\right]$. However, a significant interaction effect could be observed in two conditions: (i) group $\mathrm{x}$ movement type [left eye: $F_{(1,187.1)}=3.3, p=0.071$, right eye: $F_{(1,192.7)}=7.2$, $p=0.0079]$, which means that the higher velocity of adduction compared to abduction in HC was reversed in MS patients, resulted in a higher peak velocity in abduction compared to adduction and (ii) group $\mathrm{x}$ distance pleft eye: $F_{(1,187.1)}=7.7, p=0.006$, right eye: $\left.F_{(1,192.2)}=7.15, p=0.008\right]$, 


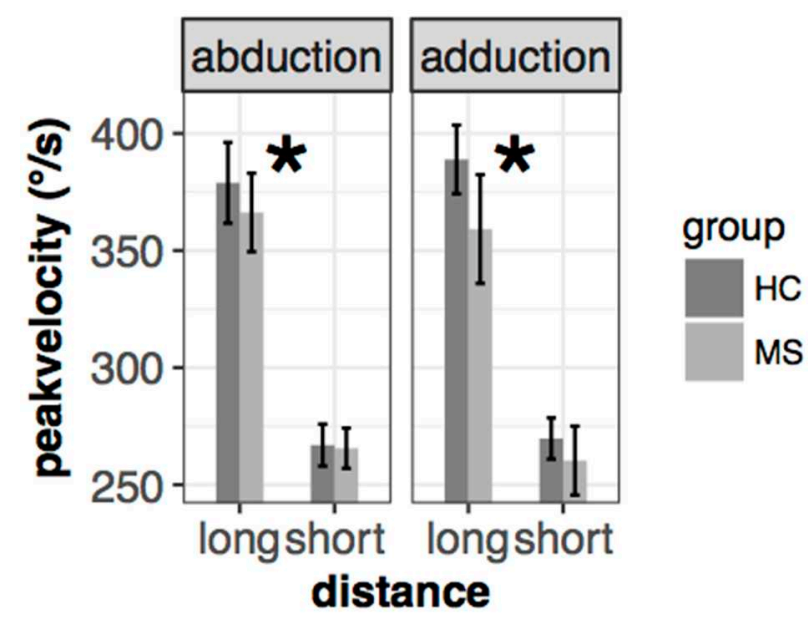

FIGURE 2 | Mean and \pm SEM of peak velocity in different conditions of the left eye in the saccade task. Significant group* distance interaction is marked with an asterisk (*). The mean and standard error are the following from left to right: $379( \pm 9) / \mathrm{s}$ vs. $366( \pm 8) / \mathrm{s}$ (abduction+long), $266( \pm 4) / \mathrm{s}$ vs. $265( \pm 4) / \mathrm{s}$ (abduction+short), 389( \pm 7$) / \mathrm{s}$ vs. 359( \pm 12$) / \mathrm{s}$ (adduction+long), 269( \pm 4$) / \mathrm{s}$ vs. $260( \pm 7) /$ s (adduction+short) for $\mathrm{HC}$ and MS, respectively.

which means that the slower peak velocity in the closer cue condition in HC group was slightly smaller in the MS group (Figure 2).

Anti-saccade peak velocity was not different between the two groups $\left[F_{(1,64.8)}=0.12, p=0.73\right]\left[\right.$ mean $( \pm \mathrm{sd}): 265( \pm 46)^{\circ} / \mathrm{s}$ vs. $268( \pm 41)^{\circ} / \mathrm{s}$ for $\mathrm{HC}$ and MS, respectively] and no interaction effects could be observed.

Difference between prosaccade and anti-saccade peak velocity could be observed in a group level. MS group had lower difference in peak velocity around $20 / \mathrm{s}\left[t_{(64)}=2.13, p=0.037\right][$ mean $( \pm \mathrm{sd})$ : $60( \pm 33)^{\circ} / \mathrm{s}$ vs. $42( \pm 37)^{\circ} / \mathrm{s}$ for $\mathrm{HC}$ and $\mathrm{MS}$, respectively], however, this difference was not survived scaling for amplitude $\left[t_{(64)}=0.07, p=0.95\right]$.

Moreover, EDSS scores positively correlated with peak velocity difference (Spearman's rho: $0.4, p=0.024$ ). The higher clinical disability related to higher peak velocity difference between the two tasks.

\section{Gain}

In the prosaccade task, MS group had smaller gain in all conditions. Therefore, they performed slightly hypometric saccades compared to HC. However, this difference did not reach a significant level $\left[F_{(1,65.8)}=2.24, p=0.14\right]$ [mean $( \pm \mathrm{sd}): 0.932( \pm 0.046)$ vs. $0.913( \pm 0.049)$ for HC and MS, respectively] (Figure 3).

Gain in the anti-saccade task did not differ between the two groups $\left[F_{(1,63.6)}=0.01, p=0.92\right]$.

\section{Antisaccade Performance}

There was a marked difference between the two groups in the anti-saccade performance. The HC group reached more than $80 \%( \pm 12.2 \%)$ accuracy while the MS group obtained only

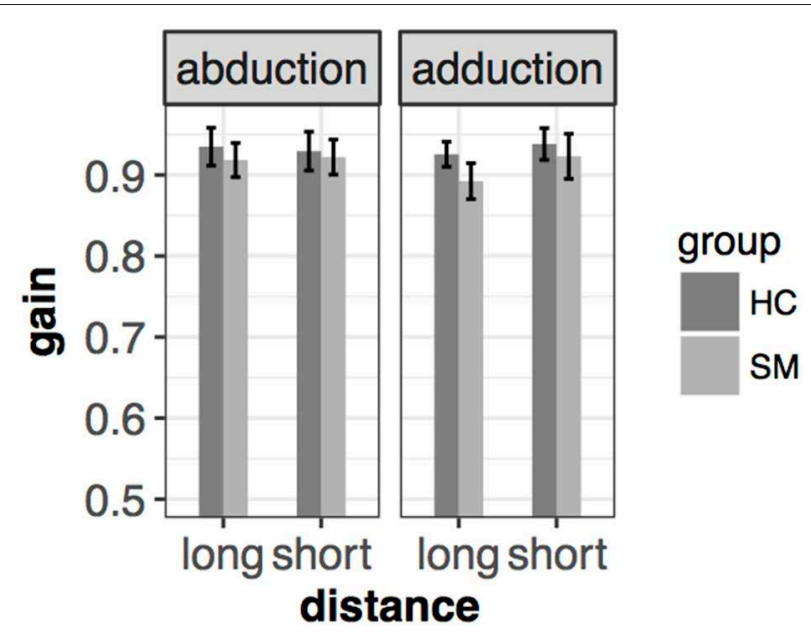

FIGURE 3 | Mean and \pm SEM of gain in different conditions of the left eye in the saccade task. Slight hypometria could be detected, however, this difference was not significant. Mean and standard error are the following from left to right: $0.935( \pm 0.012)$ vs. $0.918( \pm 0.11)$ (abduction+long), $0.929( \pm 0.012)$ vs. 0.922( \pm 0.011$)$ (abduction+short), 0.926( \pm 0.008$)$ vs. 0.89( \pm 0.011$)$ (adduction+long), 0.938( \pm 0.01 ) vs. (adduction+short) for $\mathrm{HC}$ and MS, respectively.

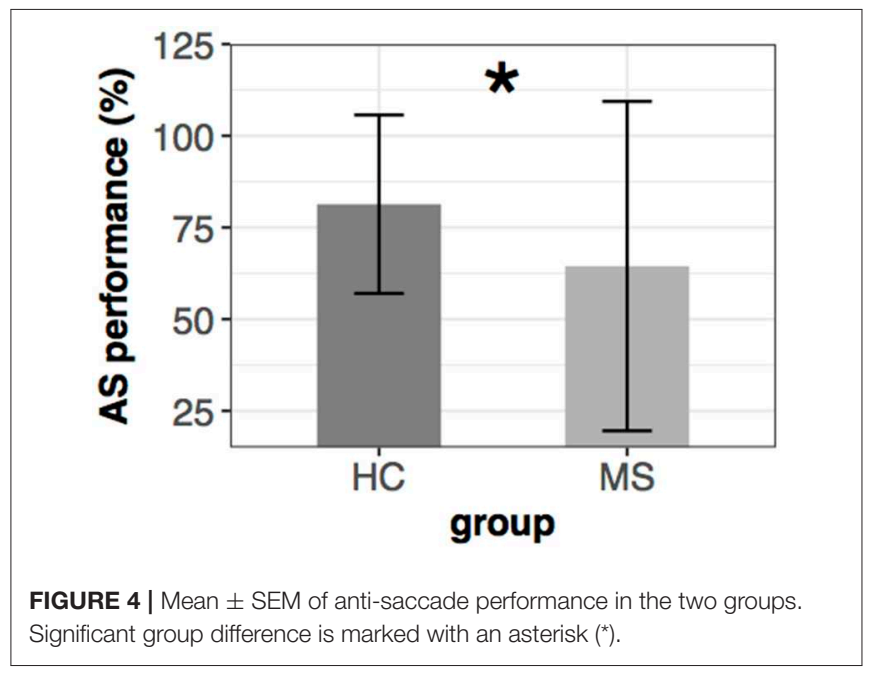

$64 \%( \pm 22.5 \%)$. (Wilcoxon-Mann-Whitney test: $\left[U_{34,33}=837\right.$, $p<0.001$ ] (Figure 4).

\section{Dyconjugacy Index}

The dysconjugacy index derived from peak velocities of the eyes can detect clinically not detectable internuclear ophthalmoparesis (INO). A $Z$-value higher than the highest control subject's $z$-value +2 was determined as threshold for subclinical INO (10). Based on this threshold five patients were classified as having subclinical INO. Patient \#49 had INO in both directions, while patient \#39, \#37, and \#17 had only in the left direction and patient \#18 only in the right direction (Figure 5). 


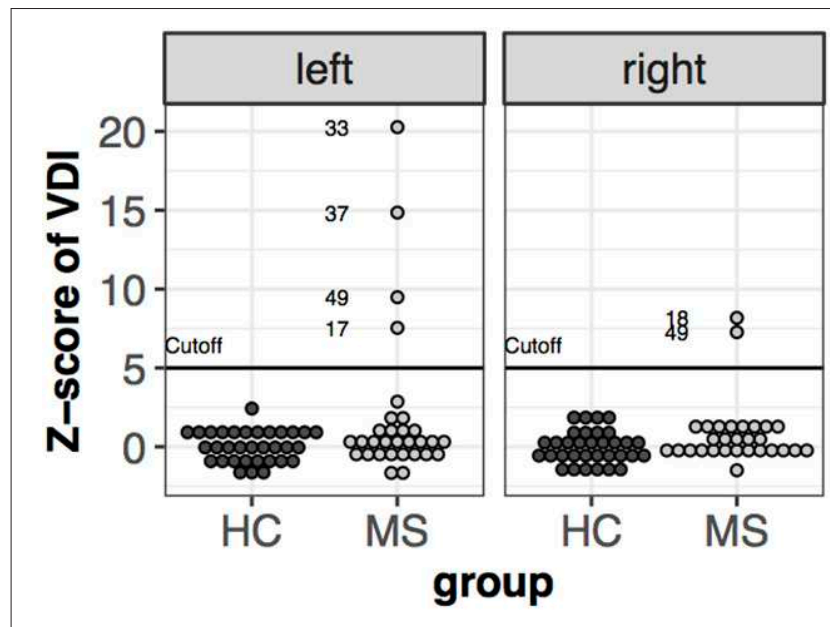

FIGURE 5 | Z-scores of velocity dysconjugacy index individually, Subjects' value higher than the cut-off are labeled.

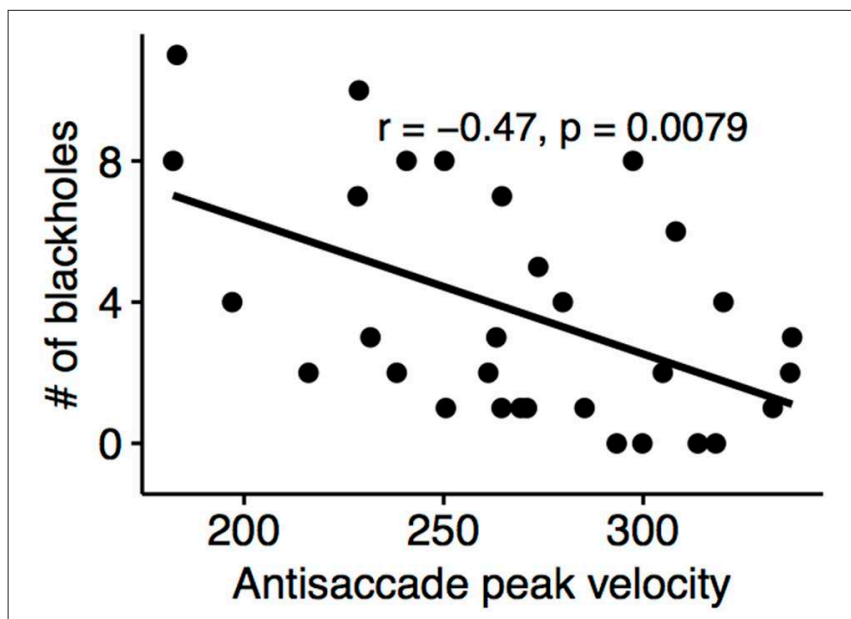

FIGURE 6 | Antisaccade peak velocity of the left eye negatively correlated with black-hole count.

\section{Correlation of Eye Movement Deficit With MRI Markers}

T2 lesion burden or lesion location did not show significant correlation with any of the measured MRI parameters.

A positive correlation were detected between anti-saccade latency and the number of black-holes (Spearman's rho: 0.45, $p=0.011)$ and negative correlation between the anti-saccade peak velocity and the number of black-holes (Spearman's rho: $-0.47, p<0.01$ ) (Figure 6).

There were no significant correlation between white matter volume with any of the measured eye movement parameters.

The VBM analysis revealed that anti-saccade peak velocity correlated with gray matter density in parietal areas (Figure 7). That is, smaller anti-saccade peak velocity was associated with lower gray matter densities in the left parietal areas.

No other eye movement parameters showed correlation with gray matter density.

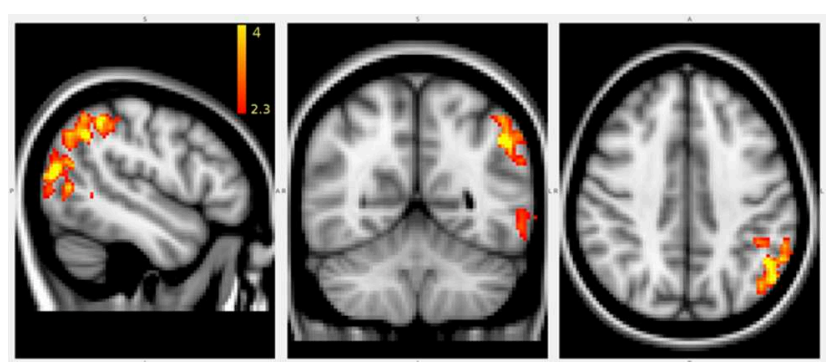

FIGURE 7 | Results of VBM analysis. Marked voxels positively correlated with anti-saccade peak velocity. The error bar represents different $Z$-value after cluster based thresholding. Disease duration and age were used as cofounders.

\section{DISCUSSION}

In our study we investigated visually guided prosaccade and anti-saccade task performance in MS patients and their possible association with focal brain alterations. Out of several eye movement parameters, we found significantly increased latency in the prosaccade task and significantly worse performance in the anti-saccade task in MS patients. The detailed examination of conjugated eye movements revealed 5 subclinical INO cases.

As regarding the MRI parameters, the peak velocity and latency of the anti-saccade movement correlated with the number of black-holes, but none of the eye movement parameters were associated with the T2 lesion burden or location. Most importantly, local gray matter atrophy in the left inferioparietal lobule and temporo-parietal junction correlated with anti-saccade peak velocity.

Oculomotor alterations found with various paradigms such as visually guided saccade, prosaccade, anti-saccade, memory guided saccade and endogenous cued saccade are common in MS $(1,3,10,24-31)$. In agreement with our findings, Clough et al. found that saccade latency is prolonged in clinically definitive MS patients (24). In the same cohort, latency increases with longer disease duration. In another study, the MS group has longer saccade latency in the presence of a distractor stimulus (29). Previous studies also showed that the performance is deteriorated mainly in more cognitively demanding saccade tasks $(3,24-$ $28,31)$. Antisaccade performance is deteriorated and associated with cognitive performance $(24,28,31)$. Fielding claims that this alteration spares the reflexive part of the saccades. In our investigation the anti-saccade peak velocity, but not that of the parameters of the saccade task correlated with number of blackholes and focal gray matter atrophy in the temporo-parietal region. Prolonged latency of prosaccade could mirror the delayed initiation of saccades. The prolonged latency of anti-saccades however, might reflect a prolonged volitional decision process or a delayed initiation of saccade in the opposite direction or both (32). The difference might relates to the time, which is not necessary for the reflexive part such as inhibition or vector transformation (33). Hence the correlations we have found are mainly reflecting the higher order cognitive processes 
of eye movements rather the reflexive parts. Interestingly, no correlation was found between any of the MRI parameters and the anti-saccade performance. The non-reflexive part of the antisaccades might be dysfunctional, leading to an error. While if it is delayed but to a level that is not sufficient to make an error it could only be investigated via its delayed latency. This could especially be important in multiple sclerosis, in which demyelination, and slowed conduction is a key feature of the disease.

Saccades could be a potential marker to follow-up cognitive alterations in MS patients because it has been shown that various saccade performances are associated with cognition $(24,25,27)$. Based on these observations, prolonged latency could reflect damage to networks associated with motor or cognitive control.

While several studies showed alterations of oculomotor performance in MS, the background of such alterations is not entirely clear. Clinically detectable oculomotor symptoms show correlation with certain infratentorial lesions (9), but in our investigation, subclinical eye movement deficit did not correlate with T2 hyperintense lesion load, which is congruent with the result of a previous study (31). It is in agreement with our earlier investigation in that lesion load or location only modestly correlate with clinical symptoms (13), whereas persistent black-holes show better correlation with clinical and cognitive functioning in MS $(34,35)$. Accordingly, the T1 hypointense lesion burden correlated with the anti-saccade velocity and latency in our study. Brain atrophy showed better correlation with clinical and cognitive disability (36). In particular, cerebellar atrophy was associated with anti-saccade error (31). In our study, gray matter atrophy measures correlated with eye movement deficits globally as well as locally. Black holes and atrophy seem to jointly relate to anti-saccade alterations. Several studies found correlation between T1 black-hole lesions and atrophy, but no similar relationship was revealed for T2 hyperintense lesions $(37,38)$. Cellular damage could be observed in both cases $(39,40)$. In addition, both measures correlate well with clinical disability, better than T2 lesion load (41).

Saccade peak velocity is affected by multiple cognitive functions [arousal (42) and mental workload (43)]. In our study, focal gray matter volume variability showed correlation with anti-saccade peak velocity in the left inferior parietal lobule, left temporo-parietal junction and in the putative left V5/MT motion sensitive visual region (44). These parietal regions are identical to those frequently implicated in attention tasks (45). In their seminal paper, Corbetta et al. in a remarkably similar paradigm found activation in the intraparietal sulcus during sustained attention and in the right temporo-parietal junction when a target was detected, particularly at an unattended location (4).

\section{REFERENCES}

1. Mastaglia FL, Black JL, Collins DW. Quantitative studies of saccadic and pursuit eye movements in multiple sclerosis. Brain. (1979) 102:817-34.

2. Servillo G, Renard D, Taieb G, Labauge P, Bastide S, Zorzon M, et al. Bedside tested ocular motor disorders in multiple sclerosis patients. Multiple Sclerosis Int. (2014) 2014:732329. doi: 10.1155/2014/732329
These two conditions correspond to the top-down and bottomup attentional subsystems. Moreover, the parietal cortex has its direct connection to the superior colliculus (6-8), and the pontine nuclei as well (46). Damage to these perisylvian regions was also implicated in neglect (47). Visuo-spatial neglect in MS patients has been described, but no associated structural damage has been found so far (48). Interestingly, the right temporoparietal junction is implicated in target detection, but in our study the atrophy of the left temporo-parietal junction was associated.

Alternatively, parietal region has a potential role in saccades. The parietal eye field and posterior parietal cortex are involved in saccade generation (49) and visuospatial attention (45). Moreover, human (50) and animal (51) studies suggested that this region has a role in the vector inversion process, which is a crucial step in anti-saccades.

In conclusion, saccades are substantially affected in MS patients, which reflected in several behavior parameters. Global and focal gray matter alterations are associated with brain areas important in cognitive functions, such as attention.

\section{ETHICS STATEMENT}

This study was carried out in accordance with the recommendations of the Medical Research Council National Scientific and Ethical Committee (ETT TUKEB) with written informed consent from all subjects. All subjects gave written informed consent in accordance with the Declaration of Helsinki. The protocol was approved by the National Institute of Pharmacy and Nutrition (000002/2016/OTIG).

\section{AUTHOR CONTRIBUTIONS}

$\mathrm{BK}$, NS, and ZK: designed the study. BK, BH, BB, KK, PF, AK, DV, ET, and NS: collected and organized the database. ZK, KB, and $L V$ : recruitment and interpretation of the data. $B K$ and $\mathrm{BH}$ : performed the statistical analysis. $\mathrm{BK}$ and $\mathrm{ZK}$ : wrote the manuscript. All authors contributed to manuscript revision, read and approved the submitted version.

\section{FUNDING}

This paper was supported by the MTA-SZTE Neuroscience Research Group, the National Brain Research Programs (Grant No. KTIA_13_NAP-A-II/20) and an EFOP grant (EFOP3.6.1-16-2016-00008). BK was supported by the UNKP-183 New National Excellence Program of the Ministry of Human Capacities. 
5. Pierrot-Deseilligny C, Müri RM, Ploner CJ, Gaymard B, RivaudPéchoux S. Cortical control of ocular saccades in humans: a model for motricity. Prog Brain Res. (2003) 142:3-17. doi: 10.1016/S0079-6123(03) 42003-7

6. Cerkevich CM, Lyon DC, Balaram P, Kaas JH. Distribution of cortical neurons projecting to the superior colliculus in macaque monkeys. Eye Brain. (2014) 2014:121-37. doi: 10.2147/EB.S53613

7. Rushworth MF, Behrens TE, Johansen-Berg H. Connection patterns distinguish 3 regions of human parietal cortex. Cerebral Cortex. (2006) 16:1418-30. doi: 10.1093/cercor/bhj079

8. Pare M, Wurtz RH. Monkey posterior parietal cortex neurons antidromically activated from superior colliculus. J Neurophysiol. (1997) 78:3493-7. doi: 10.1152/jn.1997.78.6.3493

9. Serra A, Chisari CG, Matta M. Eye movement abnormalities in multiple sclerosis: pathogenesis, modeling, and treatment. Front Neurol. (2018) 9:31. doi: 10.3389/fneur.2018.00031

10. Frohman EM, Frohman TC, O'Suilleabhain P, Zhang H, Hawker $\mathrm{K}$, Racke MK, et al. Quantitative oculographic characterisation of internuclear ophthalmoparesis in multiple sclerosis: the versional dysconjugacy index Z score. J Neurol Neurosurg Psychiatry. (2002) 73:51-5. doi: 10.1136/jnnp.73.1.51

11. Agarwal M, Ulmer JL, Chandra T, Klein AP, Mark LP, Mohan S. Imaging correlates of neural control of ocular movements. Eur Radiol. (2016) 26:2193205. doi: 10.1007/s00330-015-4004-9

12. Kincses ZT, Tóth E, Fricska-Nagy ZS, Füvesi J, Rajda C, Bencsik K, et al. The role of MRI in measuring the effectivity of disease modifying treatments II. Ideggyogy Sz. (2018) 71:81-8. doi: 10.18071/isz.71.0081

13. Kincses ZT, Ropele S, Jenkinson M, Khalil M, Petrovic K, Loitfelder $\mathrm{M}$, et al. Lesion probability mapping to explain clinical deficits and cognitive performance in multiple sclerosis. Mult Scler. (2011) 17:6819. doi: $10.1177 / 1352458510391342$

14. Jenkinson $M$, Smith S. A global optimisation method for robust affine registration of brain images. Med Image Anal. (2001) 5:143-56. doi: 10.1016/S1361-8415(01)00036-6

15. Andersson JLR, Jenkinson M, Smith, S. Non-Linear Optimisation. FMRIB Technical report. Oxford (2007).

16. Ashburner J, Friston KJ. Voxel-based morphometry-the methods. NeuroImage. (2000) 11(Pt. 1):805-21. doi: 10.1006/nimg.2000.0582

17. Good CD, Johnsrude IS, Ashburner J, Henson RN, Friston KJ, Frackowiak RS. A voxel-based morphometric study of ageing in 465 normal adult human brains. NeuroImage. (2001) 14(Pt 1):21-36. doi: 10.1006/nimg.2001. 0786

18. Smith SM, Jenkinson M, Woolrich MW, Beckmann CF, Behrens TE, Johansen-Berg $\mathrm{H}$, et al. Advances in functional and structural MR image analysis and implementation as FSL. NeuroImage. (2004) 23(Suppl. 1):S20819. doi: 10.1016/j.neuroimage.2004.07.051

19. Smith SM. Fast robust automated brain extraction. Hum Brain Mapp. (2002) 17:143-55. doi: 10.1002/hbm.10062

20. Zhang Y, Brady M, Smith S. Segmentation of brain MR images through a hidden Markov random field model and the expectation-maximization algorithm. IEEE Trans Med Imaging. (2001) 20:45-57. doi: 10.1109/42. 906424

21. R_core_team. R. A Language and Environment for Statistical Computing. R Foundation for Statistical Computing. Vienna (2017).

22. Bates D, Mächler M, Bolker B, Walker S. Fitting linear mixed-effects models using lme4. J Stat Softw. (2015) 67:1-48. doi: 10.18637/jss.v067.i01

23. Weisberg S. An $\{R\}$ Companion to Applied Regression, 2nd ed. Thousand Oaks, CA: Sage (2011).

24. Clough M, Millist L, Lizak N, Beh S, Frohman TC, Frohman EM, et al. Ocular motor measures of cognitive dysfunction in multiple sclerosis I: inhibitory control. J Neurol. (2015) 262:1130-7. doi: 10.1007/s00415-015-7645-3

25. Clough M, Mitchell L, Millist L, Lizak N, Beh S, Frohman TC, et al. Ocular motor measures of cognitive dysfunction in multiple sclerosis II: working memory. J Neurol. (2015) 262:1138-47. doi: 10.1007/s00415-01 5-7644-4

26. Fielding J, Kilpatrick T, Millist L, Clough M, White O. Longitudinal assessment of antisaccades in patients with multiple sclerosis. PloS ONE. (2012) 7:e30475. doi: 10.1371/journal.pone.0030475
27. Fielding J, Kilpatrick T, Millist L, White O. Multiple sclerosis: cognition and saccadic eye movements. J Neurol Sci. (2009) 277:32-6. doi: 10.1016/j.jns.2008.10.001

28. Fielding J, Kilpatrick T, Millist L, White O. Antisaccade performance in patients with multiple sclerosis. Cortex. (2009) 45:900-3. doi: 10.1016/j.cortex.2009.02.016

29. Fielding J, Kilpatrick T, Millist L, White O. Control of visually guided saccades in multiple sclerosis: disruption to higher-order processes. Neuropsychologia. (2009) 47:1647-53. doi: 10.1016/j.neuropsychologia.2009. 01.040

30. Finke C, Pech LM, Sommer C, Schlichting J, Stricker S, Endres M, et al. Dynamics of saccade parameters in multiple sclerosis patients with fatigue. J Neurol. (2012) 259:2656-63. doi: 10.1007/s00415-012-6565-8

31. Kolbe SC, Kilpatrick TJ, Mitchell PJ, White O, Egan GF, Fielding J. Inhibitory saccadic dysfunction is associated with cerebellar injury in multiple sclerosis. Hum Brain Mapp. (2014) 35:2310-9. doi: 10.1002/hbm.22329

32. Chen YF, Chen T, Tsai TT. Analysis of volition latency on antisaccadic eye movements. Med Eng Phys. (1999) 21:555-62

33. Munoz DP, Everling S. Look away: the anti-saccade task and the voluntary control of eye movement. Nat Rev Neurosci. (2004) 5:21828. doi: $10.1038 / \mathrm{nrn} 1345$

34. Giorgio A, Stromillo ML, Bartolozzi ML, Rossi F, Battaglini M, De Leucio A, et al. Relevance of hypointense brain MRI lesions for long-term worsening of clinical disability in relapsing multiple sclerosis. Mult Scler. (2014) 20:2149. doi: 10.1177/1352458513494490

35. Zivadinov R, Leist TP. Clinical-magnetic resonance imaging correlations in multiple sclerosis. J Neuroimaging. (2005) 15(Suppl. 4):10S-21. doi: 10.1177/1051228405283291

36. Kincses ZT, Toth E, Banko N, Vereb D, Szabo N, Csete G, et al. Grey matter atrophy in patients suffering from multiple sclerosis. Ideggyogy $S z$. (2014) 67:293-300.

37. Sailer M, Losseff NA, Wang L, Gawne-Cain ML, Thompson AJ, Miller DH. T1 lesion load and cerebral atrophy as a marker for clinical progression in patients with multiple sclerosis. A prospective 18 months follow-up study. Eur J Neurol. (2001) 8:37-42. doi: 10.1046/j.1468-1331.2001.00147.x

38. Paolillo A, Pozzilli C, Gasperini C, Giugni E, Mainero C, Giuliani S, et al. Brain atrophy in relapsing-remitting multiple sclerosis: relationship with 'black holes', disease duration and clinical disability. J Neurol Sci. (2000) 174:85-91. doi: 10.1016/S0022-510X(00)00259-8

39. van Walderveen MA, Kamphorst W, Scheltens P, van Waesberghe JH, Ravid R, Valk J, et al. Histopathologic correlate of hypointense lesions on T1-weighted spin-echo MRI in multiple sclerosis. Neurology. (1998) 50:1282-8.

40. Wegner C, Esiri MM, Chance SA, Palace J, Matthews PM. Neocortical neuronal, synaptic, and glial loss in multiple sclerosis. Neurology. (2006) 67:960-7. doi: 10.1212/01.wnl.0000237551.26858.39

41. Rocca MA, Comi G, Filippi M. The role of T1-weighted derived measures of neurodegeneration for assessing disability progression in multiple sclerosis. Front Neurol. (2017) 8:433. doi: 10.3389/fneur.2017.00433

42. Di Stasi LL, Catena A, Canas JJ, Macknik SL, Martinez-Conde S. Saccadic velocity as an arousal index in naturalistic tasks. Neurosci Biobehav Rev. (2013) 37:968-75. doi: 10.1016/j.neubiorev.2013.03.011

43. Di Stasi LL, Renner R, Staehr P, Helmert JR, Velichkovsky BM, Canas JJ, et al. Saccadic peak velocity sensitivity to variations in mental workload. Aviat Space Environ Med. (2010) 81:413-7. doi: 10.3357/ASEM. 2579.2010

44. Dumoulin SO, Bittar RG, Kabani NJ, Baker CL Jr., Le Goualher G, Bruce Pike G, et al. A new anatomical landmark for reliable identification of human area V5/MT: a quantitative analysis of sulcal patterning. Cereb Cortex. (2000) 10:454-63. doi: 10.1093/cercor/10.5.454

45. Corbetta M, Shulman GL. Control of goal-directed and stimulus-driven attention in the brain. Nat Rev Neurosci. (2002) 3:201-15. doi: 10.1038/ nrn755

46. Anja K.E. Horn CA. Reticular formation: eye movements, gaze and blinks. In: Jürgen K, Mai GP, editors. The Human Nervous System, 3rd ed. Cambridge, MA: Academic Press (2012). p. 328-66.

47. Karnath HO, Rorden C. The anatomy of spatial neglect. Neuropsychologia. (2012) 50:1010-7. doi: 10.1016/j.neuropsychologia.2011.06.027 
48. Gilad R, Sadeh M, Boaz M, Lampl Y. Visual spatial neglect in multiple sclerosis. Cortex. (2006) 42:1138-42. doi: 10.1016/S0010-9452(08)70226-0

49. Pierrot-Deseilligny C, Milea D, Muri RM. Eye movement control by the cerebral cortex. Curr Opin Neurol. (2004) 17:17-25. doi: 10.1097/01.wco.0000113942.12823.e0

50. Everling S, Spantekow A, Krappmann P, Flohr H. Event-related potentials associated with correct and incorrect responses in a cued antisaccade task. Exp Brain Res. (1998) 118:27-34.

51. Gottlieb J, Goldberg ME. Activity of neurons in the lateral intraparietal area of the monkey during an antisaccade task. Nat Neurosci. (1999) 2:90612. doi: $10.1038 / 13209$
Conflict of Interest Statement: The authors declare that the research was conducted in the absence of any commercial or financial relationships that could be construed as a potential conflict of interest.

Copyright $\odot 2019$ Kincses, Hérák, Szabó, Bozsik, Faragó, Király, Veréb, Tóth, Kocsis, Bencsik, Vécsei and Kincses. This is an open-access article distributed under the terms of the Creative Commons Attribution License (CC BY). The use, distribution or reproduction in other forums is permitted, provided the original author(s) and the copyright owner(s) are credited and that the original publication in this journal is cited, in accordance with accepted academic practice. No use, distribution or reproduction is permitted which does not comply with these terms. 


$$
\text { II. }
$$




\title{
Brain MRI Diffusion Encoding Direction Number Affects Tract-Based Spatial Statistics Results in Multiple Sclerosis
}

\author{
Bálint Kincses iD, Tamás Spisák, Péter Faragó, András Király, Nikoletta Szabó, Dániel Veréb, Krisztián Kocsis, \\ Bence Bozsik, Eszter Tóth, László Vécsei, Zsigmond Tamás Kincses \\ From the Department of Neurology, University of Szeged, Szeged, Hungary (BK, PF, AK, NS, DV, KK, BB, ET, LV, ZTK); Department of Radiology, University of Szeged, \\ Szeged, Hungary (ZTK); MTA-SZTE Neuroscience Research Group, Szeged, Hungary (LV); Bingel Laboratory, University of Essen, Essen, Germany (TS); and Department \\ of Psychiatry, University of Szeged, Szeged, Hungary (BK).
}

\section{A B S T R A C T}

BACKGROUND AND PURPOSE: Diffusion tensor imaging (DTI) is a promising approach to detect the underlying brain pathology. These alterations can be seen in several diseases such as multiple sclerosis. Tract-based spatial statistics (TBSS) is an easy to use and robust way for analyzing diffusion data. The effect of acquisition parameters of DTI on TBSS has not been evaluated, especially the number of diffusion encoding directions (NDED), which is directly proportional with scan time.

METHODS: We analyzed a large set of DTI data of healthy controls $(N=126)$ and multiple sclerosis patients $(N=78)$. The highest NDED (60 directions) was reduced and a tensor calculation was done separately for every subset. We calculated the mean and standard deviation of DTI parameters under the white matter mask. Moreover, the FMRIB Software Library TBSS pipeline was used on DTI images with 15, 30, 45, and 60 directions to compare differences between groups. Mean DTI parameters were compared between groups as a function of NDED.

RESULTS: The mean value of FA and AD decreased with increasing number of directions. This was more pronounced in areas with smaller FA values. RD and MD were constant. The skeleton size reduced with elevating NDED along with the number of significant voxels. The TBSS analysis showed significant differences between groups throughout the majority of the skeleton and the group difference was associated with NDED.

CONCLUSION: Our results suggested that results of TBSS depended on the NDED, which should be considered when comparing DTI data with varying protocols.

Keywords: Diffusion encoding directions, diffusion tensor imaging, multiple sclerosis, tract-based spatial statistics.

Acceptance: Received December 15, 2019, and in revised form March 6, 2020. Accepted for publication March 6, 2020.

Correspondence: Address correspondence to Zsigmond Tamás Kincses, Department of Radiology, Albert Szent-Györgyi Clinical Center, University of Szeged, Semmelweis u. 6, 6725-Szeged, Hungary. E-mail: kincses.zsigmond.tamas@med.u-szeged.hu.

Acknowledgements and Disclosure: This paper was supported by an EFOP grant (EFOP-3.6.1-16-2016-00008), and a Horizon 2020 Framework Programme grant (H2020-MSCA-RISE-2016 734718). Bálint Kincses was supported by the UNKP-19-3 New National Excellence Program of the Ministry of Human Capacities. Krisztián Kocsis was supported by the UNKP-19-3-SZTE-149 New National Excellence Program of the Ministry of Human Capacities.

The copyright line for this article was changed on May 27, 2020 after original online publication.

J Neuroimaging 2020;00:1-11

DOI: $10.1111 /$ jon. 12705

\section{Introduction}

Diffusion tensor imaging (DTI) is a widely used technique to analyze tissue pathology in vivo. Several studies demonstrated structural alterations in various diseases. ${ }^{1-3}$ From the tensor model, several derived parameters are available to characterize white matter changes qualitatively. Fractional anisotropy (FA) describes how strongly the diffusion is directional in the measured volume. Mean diffusivity (MD) and the main eigenvectors represent the average diffusivity within the voxel and the diffusion along the main diffusion directions. A correct estimation of the diffusion tensor requires at least six noncollinear diffusion directions and an extra nondiffusion-weighted image. Because diffusion-weighted measurement is typically characterized by relatively small signal-to-noise ratio (SNR), one could attain higher SNR with increasing the number of diffusion encoding directions (NDED). To achieve tensor orientation and anisotropy-independent SNR, one should use a uniform distribution of the encoding directions. ${ }^{4}$ Moreover, the optimal NDED is critical for SNR and scan time. More directions increase $\mathrm{SNR}^{5}$ but prolong the measurement. Several theoretical and practical studies demonstrated the NDED dependency of the obtained diffusion tensor values and propose an optimum NDED which, depending on the optimization metric, ranges between 6 and $62 .{ }^{5-12}$

Although there is a considerable body of research in this topic, there are still outstanding issues to be investigated. Most of these studies evaluated data from healthy controls $(\mathrm{HC})$ or used simulated data. ${ }^{5-12}$ In vivo studies include the physiological effects (movements, cardiorespiratory effects, etc) and patient-related special features (eg, different in-scanner motion). In addition, special analytical methods could be sensitive to the bias of the tensor value. The widely used tract-based spatial statistics (TBSS) is an easy to use and robust method to analyze DTI images. ${ }^{13}$ The approach

This is an open access article under the terms of the Creative Commons Attribution-NonCommercial-NoDerivs License, which permits use and distribution in any medium, provided the original work is properly cited, the use is non-commercial and no modifications or adaptations are made. 
Table 1. Clinical and Demographical Data of Participants

\begin{tabular}{|c|c|c|c|c|}
\hline & $\begin{array}{l}\text { Number of } \\
\text { participants } \\
\text { (female) }\end{array}$ & Age (year) & EDSS & $\begin{array}{c}\text { Disease } \\
\text { duration } \\
\text { (year) }\end{array}$ \\
\hline MS & $78(55)$ & $38.6( \pm 9.9)$ & $1(0-6.5)$ & $8.7( \pm 6.8)$ \\
\hline $\mathrm{HC}$ & $126(57)$ & $31( \pm 10.2)$ & - & - \\
\hline \multicolumn{5}{|c|}{$\begin{array}{l}\text { Note. Age and disease duration are mean } \pm \text { standard deviation. Median expanded } \\
\text { disability status scale and the range are shown. EDSS = expanded disability status } \\
\text { scale. }\end{array}$} \\
\hline \multicolumn{5}{|c|}{$\begin{array}{l}\text { Table 2. The Mean of DTI Parameters in the Individual White Matter } \\
\text { Mask for Different Subset of Encoding Directions for Healthy } \\
\text { Controls }\end{array}$} \\
\hline NDED & AD & FA & MD & RD \\
\hline 10 & $1.20 \times 10^{-3}$ & $4.86 \times 10^{-1}$ & $7.58 \times 10^{-4}$ & $5.39 \times 10^{-4}$ \\
\hline 15 & $1.18 \times 10^{-3}$ & $4.69 \times 10^{-1}$ & $7.60 \times 10^{-4}$ & $5.48 \times 10^{-4}$ \\
\hline 20 & $1.18 \times 10^{-3}$ & $4.64 \times 10^{-1}$ & $7.62 \times 10^{-4}$ & $5.52 \times 10^{-4}$ \\
\hline 25 & $1.18 \times 10^{-3}$ & $4.61 \times 10^{-1}$ & $7.61 \times 10^{-4}$ & $5.52 \times 10^{-4}$ \\
\hline 30 & $1.18 \times 10^{-3}$ & $4.59 \times 10^{-1}$ & $7.61 \times 10^{-4}$ & $5.53 \times 10^{-4}$ \\
\hline 35 & $1.17 \times 10^{-3}$ & $4.58 \times 10^{-1}$ & $7.59 \times 10^{-4}$ & $5.53 \times 10^{-4}$ \\
\hline 40 & $1.17 \times 10^{-3}$ & $4.58 \times 10^{-1}$ & $7.58 \times 10^{-4}$ & $5.52 \times 10^{-4}$ \\
\hline 45 & $1.17 \times 10^{-3}$ & $4.57 \times 10^{-1}$ & $7.58 \times 10^{-4}$ & $5.52 \times 10^{-4}$ \\
\hline 50 & $1.17 \times 10^{-3}$ & $4.57 \times 10^{-1}$ & $7.58 \times 10^{-4}$ & $5.52 \times 10^{-4}$ \\
\hline 55 & $1.17 \times 10^{-3}$ & $4.56 \times 10^{-1}$ & $7.58 \times 10^{-4}$ & $5.53 \times 10^{-4}$ \\
\hline 60 & $1.17 \times 10^{-3}$ & $4.56 \times 10^{-1}$ & $7.57 \times 10^{-4}$ & $5.52 \times 10^{-4}$ \\
\hline
\end{tabular}

$\mathrm{AD}=$ axial diffusivity $; \mathrm{FA}=$ fractional anisotropy $\mathrm{MD}=$ mean diffusivity $; \mathrm{RD}$ $=$ radial diffusivity; $\mathrm{NDED}=$ number of diffusion encoding directions.

resolves several problems of the voxelwise analysis of the DTI data, mainly arising from the misalignment of the multisubject data. The method utilizes a fine-tuned nonlinear registration and projection onto an alignment-invariant tract representation (the mean FA skeleton) that improves the sensitivity and interpretability of the multisubject DTI studies. Since the publication of the original study, an enormous number of studies used this approach (1,737 article cited it according to PubMed in 07/2019). As the method concentrates on the center of the tracks (local maxima of the FA), where the white matter is most uniformly organized, NDED might have specific implications for this analysis technique. However, no previous study investigated the effect of NDED on the TBSS analysis according to our knowledge.

In the current investigation, our aim was to evaluate the effect of the NDED on the performance of TBSS in a realworld patient population. We chose multiple sclerosis (MS) as a target disease, because demyelination is a key feature of the pathology, which is on a scale that is detectable with DTI. ${ }^{14,15}$ The effect of NDED on TBSS' performance between healthy and MS group is questionable.

\section{Methods \\ Subjects}

One hundred and twenty-six $\mathrm{HC}$ and seventy-eight MS patients were recruited between 2009 and 2017 and a total of 204 scans were used in our analysis. We used the HC group to evaluate the effect of diffusion encoding directions on areas with different FA values. The age of the participants was between 21 and 60 at the time of the magnetic resonance imaging (MRI) scan. Exclusion criteria for the $\mathrm{HC}$ subjects were the presence of any psychiatric or neurological disease, as well as any chronic
Table 3. The Mean Bias of DTI Parameters from the Reference, 60 Directions Image in White Matter Mask in Healthy Controls

\begin{tabular}{lcccc}
\hline NDED & AD & FA & MD & RD \\
\hline 10 & $2.84 \times 10^{-5}$ & $2.95 \times 10^{-2}$ & $9.37 \times 10^{-7}$ & $-1.28 \times 10^{-5}$ \\
15 & $1.62 \times 10^{-5}$ & $1.29 \times 10^{-2}$ & $2.46 \times 10^{-6}$ & $-4.27 \times 10^{-6}$ \\
20 & $1.47 \times 10^{-5}$ & $7.80 \times 10^{-3}$ & $4.67 \times 10^{-6}$ & $-2.14 \times 10^{-7}$ \\
25 & $1.02 \times 10^{-5}$ & $4.78 \times 10^{-3}$ & $3.61 \times 10^{-6}$ & $3.89 \times 10^{-7}$ \\
30 & $8.09 \times 10^{-6}$ & $3.10 \times 10^{-3}$ & $3.24 \times 10^{-6}$ & $9.60 \times 10^{-7}$ \\
35 & $5.04 \times 10^{-6}$ & $1.92 \times 10^{-3}$ & $2.02 \times 10^{-6}$ & $5.48 \times 10^{-7}$ \\
40 & $2.67 \times 10^{-6}$ & $1.60 \times 10^{-3}$ & $7.06 \times 10^{-7}$ & $-1.59 \times 10^{-7}$ \\
45 & $2.35 \times 10^{-6}$ & $8.34 \times 10^{-4}$ & $9.68 \times 10^{-7}$ & $3.33 \times 10^{-7}$ \\
50 & $1.63 \times 10^{-6}$ & $7.11 \times 10^{-4}$ & $5.32 \times 10^{-7}$ & $1.03 \times 10^{-7}$ \\
55 & $1.06 \times 10^{-6}$ & $-2.94 \times 10^{-5}$ & $6.67 \times 10^{-7}$ & $5.48 \times 10^{-7}$ \\
\hline
\end{tabular}

$\mathrm{AD}=$ axial diffusivity $; \mathrm{FA}=$ fractional anisotropy $\mathrm{MD}=$ mean diffusivity $\mathrm{RD}$ $=$ radial diffusivity; $\mathrm{NDED}=$ number of diffusion encoding directions.

conditions. An experienced neuroradiologist (8 years of experience) reviewed all images and in case of any abnormality the subject was excluded from further analysis. The MS subjects were enrolled from our outpatient clinic. We excluded patients who did not take disease-modifying therapy on a regular basis, who experienced relapse 6 months prior to the MRI scan and who had significant brain atrophy, which affected image registration. The local ethics committee approved the study (authority number: 56/2011), and all the subjects provided written consent. Data and code are available upon request through personal correspondence after the approval of the local ethics committee.

\section{Imaging Protocol}

MRI was performed on a $1.5 \mathrm{~T}$ GE Signa Excite HDxt MR Scanner (GE Healthcare, Chalfont St. Giles, UK). T1weighted images (3D IR-FSPGR: repetition time(TR)/echo time (TE)/inversion time (TI): 10.3/4.2/450 ms, flip angle: $15^{\circ}$, Array coil Spatial Sensitivity Encoding (ASSET): 2, field of view $(\mathrm{FOV}): 25 \mathrm{~cm} \times 25 \mathrm{~cm}$, matrix: $256 \times 256$, slice thickness: $1 \mathrm{~mm}$ ) and 60 direction diffusion-weighted images with six nondiffusion-weighted reference volumes were recorded (TE: $93.8 \mathrm{~ms}$, TR: 13,500 ms, matrix: $96 \times 96$, FOV: $23 \mathrm{~cm} \times 23 \mathrm{~cm}$, flip angle: $90^{\circ}$, in-plane resolution: $2.4 \mathrm{~mm} \times 2.4 \mathrm{~mm}$, which was resampled to a $.89 \mathrm{~mm} \times .89 \mathrm{~mm}$ by the scanner, slice thickness: $2.4 \mathrm{~mm}, \mathrm{~b}: 1,000 \mathrm{~s} / \mathrm{m}^{2}$, number of excitation (NEX): 2, ASSET): 2).

\section{Data Processing}

The analyses of MR images were carried out with the tools from the FMRIB Software Library (FSL, version 5.0; Oxford Centre for Functional MRI of the Brain [FMRIB], UK;www.fmrib.ox.ac.uk/fsl) and in-house built MATLAB scripts. The following preprocessing methods were used in the diffusion image pipeline: eddy current correction and brain extraction. The FSL eddy_correct function was used to correct for subject movement and eddy currents and the FSL bet function in the nondiffusion-weighted image to eliminate nonbrain part of the image.

From the original data (60 diffusion encoding directions), the diffusion encoding directions were reduced to $n=10,15$, $20,25,30,35,40,45,50$, and 55 as follows. We used a reduction procedure to keep the uniform distribution via maximizing the total angular distribution energy. ${ }^{5}$ The angular distribution 

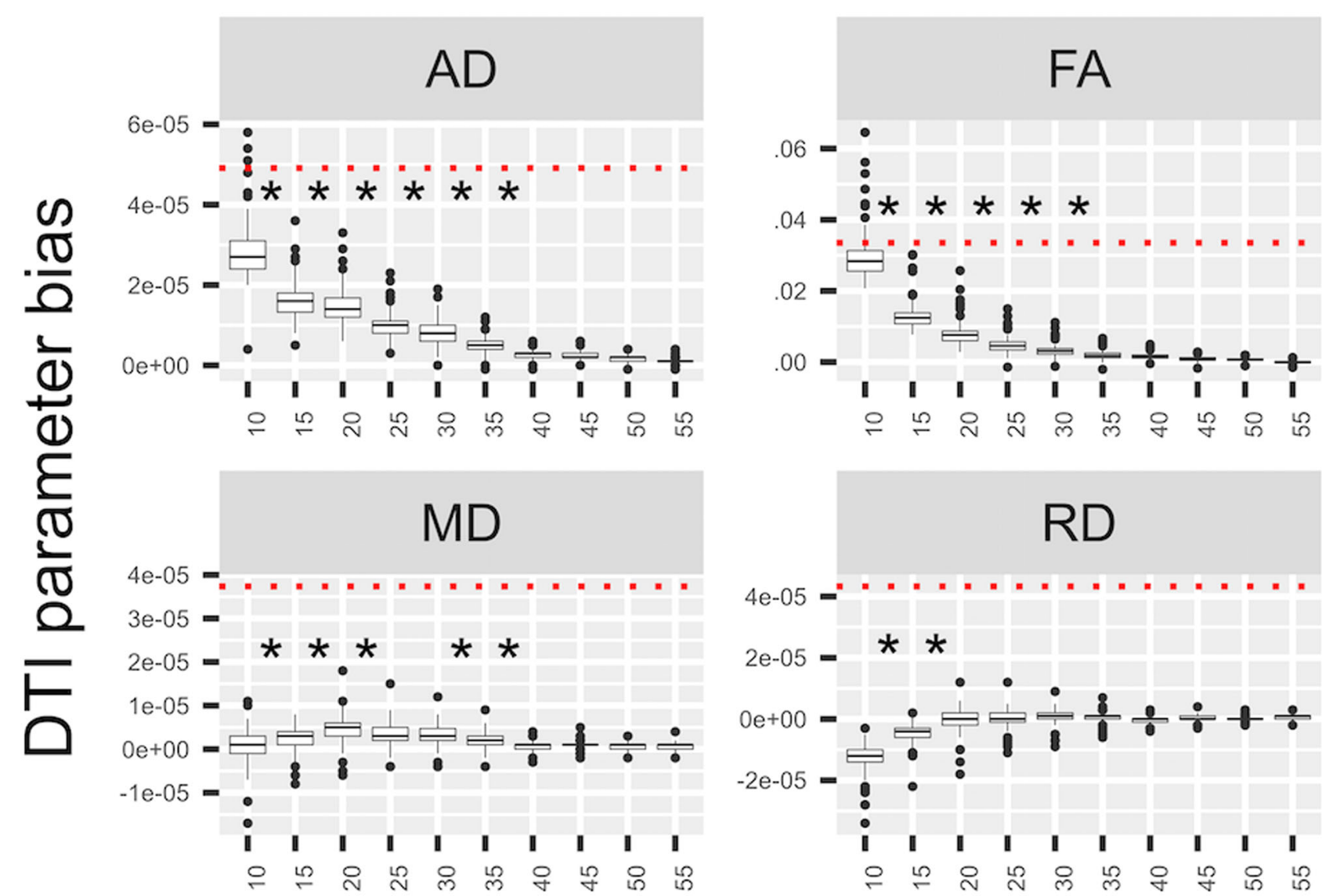

NDED

Fig 1. Bias of DTI parameters in white matter. The mean of DTI parameters' bias values under the white matter at different number of diffusion encoding directions in the $\mathrm{HC}$ group. The red dotted line shows the mean difference between the MS and HC group. Asterisks represent significant differences between groups with different directions.

energy of a pair of points on the unit sphere surface could be determined as the inverse of the sum of the squares of the least spherical distance between point a and point $b$, and the least spherical distance between point a and point b's antipodally symmetric point $\mathrm{B}(\mathrm{Eq} 1)$.

$$
E_{a, b}=\frac{1}{\left(\min _{a, b} \operatorname{dist}_{a, b}\right)^{2}+\left(\min _{a, B} \operatorname{dist}_{a, B}\right)^{2}}
$$

\section{Angular Distribution Energy of A Pair of Points}

The total angular distribution energy for a certain set of gradients can be calculated as the sum of the angular distribution energy of all pairs of gradients. The subset of gradient directions was chosen in a sequential order. ${ }^{5}$ The initial subset was one gradient and other gradients were chosen in a sequential order to maximize the total angular distribution of the set (Eq 2). The generated subset of diffusion encoding directions was selected and fed into the DTI analysis.

$$
E_{L}(N)=\sum_{a}^{N} \sum_{b}^{N} E_{a, b}
$$

\section{The Total Angular Distribution Energy of A set of Gradients}

We used the epi_reg function in FSL FLIRT ${ }^{16}$ to calculate the transformation matrix between the T1 image and diffusion space. A diffusion tensor model was fitted in each voxel with the diffusion toolbox of FSL. ${ }^{17}$ The FA maps were calculated in each group. We used FSL FAST ${ }^{18}$ on the T1-weighted images to segment the white matter from the high-resolution anatomical image. Next, the white matter tissue probability map was thresholded (.7), binarized, and transformed to the diffusion space (trilinear interpolation) with the inverse transformation matrix from epi_reg and a final threshold of 1 was used. All the individual white matter masks were visually inspected and in case of gross error the mask was recalculated and/or corrected manually. These individual white matter masks were used as region of interests (ROIs) to evaluate parameters under the white matter area. The mean and standard deviation of FA, MD, axial diffusivity (first eigenvalue, AD), and radial diffusivity (mean of the second and third eigenvalue, $\mathrm{RD}$ ) within the white matter (WM)-mask were calculated for each subject, that is, one value was determined in every subsampled data set $(10,15,20,25,30$, $35,40,45,50,55$, and 60 directions) for each subject. For every subject, we calculated the bias from its original 60-direction image for each subsampled dataset, that is, the 60 directions image 


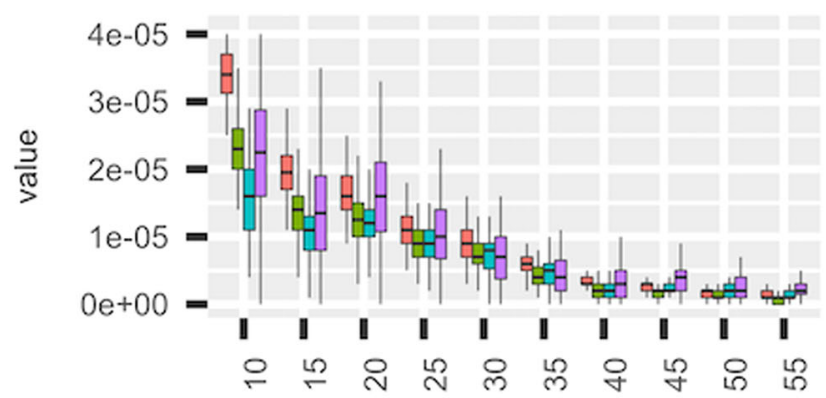

NDED

MD

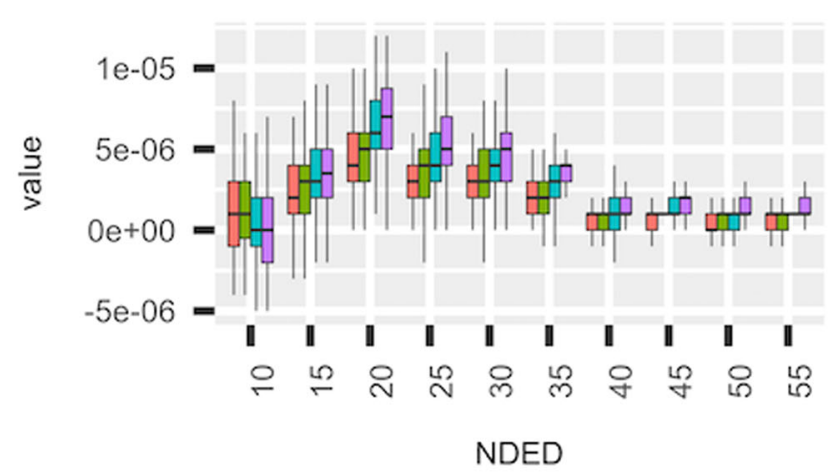

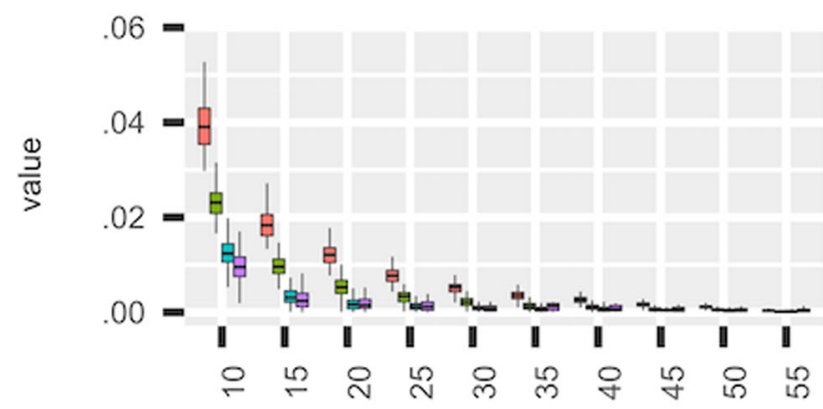

NDED

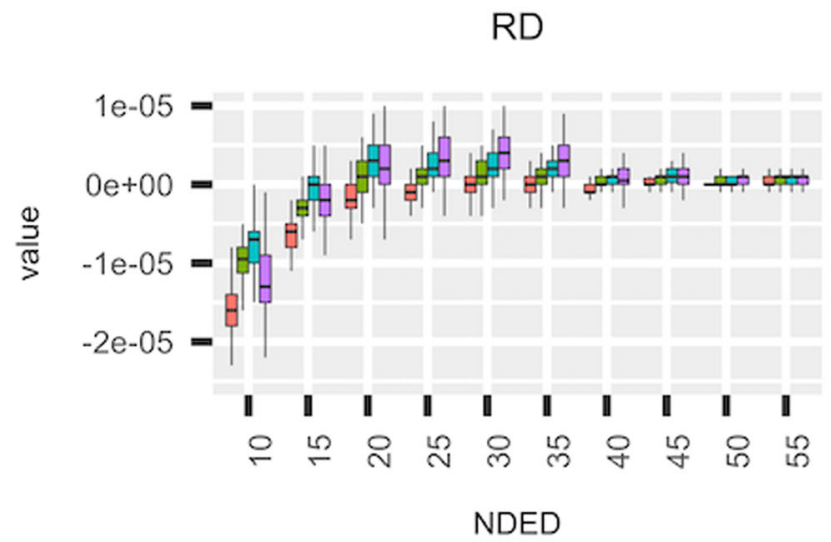

bins

FA:0.2-0.4 帨 FA:0.4-0.6

FA:0.6-0.8

FA:0.8-1

Fig 2. Bias of DTI parameters in different FA bins. The mean DTI parameter's bias from the "60 directions" image under areas of different FA values in the HC group. The four investigated DTI parameter are shown. Data points, which are out from the $95 \%$ interval, are not visualized.

was considered as a reference. We also tested for the statistical significance of the effect of directions on the mean and standard deviation for each derived parameters. Repeated measures of analysis of variance with the random effect of subjects were used within $\mathrm{R}$ studio ${ }^{19}$ as implemented in the lme4, emmeans, and car packages. ${ }^{20-22}$ The FA, MD, RD, and $\mathrm{AD}$ bias was also calculated within different white matter regions, by dividing the white matter mask into separate bins, namely, areas with .2-.4, $.4-.6, .6-.8$, and $.8-1 \mathrm{FA}$ values in the reference (60-direction) image.

To evaluate the effects of the number of diffusion directions on TBSS results, we performed a TBSS analysis separately on each DTI parameter as proposed in the FSL guideline. ${ }^{13}$ Briefly, subjects' FA images were aligned into a common space, using the nonlinear registration tool (FNIRT). A mean FA image was created and the threshold set at FA $=.3$, deriving a mean FA skeleton that represented the centers of all tracts common to the group. Each subject's aligned FA data were then projected onto this skeleton and the resulting data fed into voxel-wise statistics. We performed statistical analysis with the use of a general linear model with nonparametric permutation test for inference (5,000 permutations) with age and sex as covariates in our model. Thresholding was performed by threshold-free cluster-enhancement approach and results were corrected for multiple comparisons by controlling for the family-wise error rate. The analysis was performed in the following four subgroups of reduced directions: FA images of 15, 30, 45, and 60 directions comparing the two groups (126 HC and $78 \mathrm{MS})$. In a second analysis, the threshold in the last step of the TBSS pipeline was chosen to keep skeleton size similar (voxel count difference within .1\%), therefore FA thresholds of .3225, .307, .3028 , and .3 were used for the $15,30,45$, and 60 directions, respectively. The number of voxels in the skeleton and the number of significant voxels were calculated. Also, the mean and standard deviation of the $\mathrm{FA}, \mathrm{MD}, \mathrm{RD}$, and $\mathrm{AD}$ values in the significant voxels were investigated. Mean group differences were calculated in the significant voxels.

\section{Results}

The participants' demographical and clinical data are in Table 1 . We considered the value of the " 60 directions" group as the reference value. The mean DTI parameters and the NDEDrelated bias are listed in Tables 2 and 3 and bias is depicted in Figure 1. A decreasing trend of AD and FA could be observed with increasing NDED (Tables 2 and 3). The RD had an increasing trend and the MD was constant (Table 3 and Fig 1). Statistical analysis revealed significant main effect of directions in FA $(F(10,1250)=2,191.8, P<.0001), \operatorname{MD}(F(10,1250)=$ $114.4, P<.0001), \mathrm{AD}(F(10,1250)=1,472.6, P<.0001)$, and $\mathrm{RD}(F(10,1250)=602.1, P<.0001)$. Moreover, the 40, 35, 

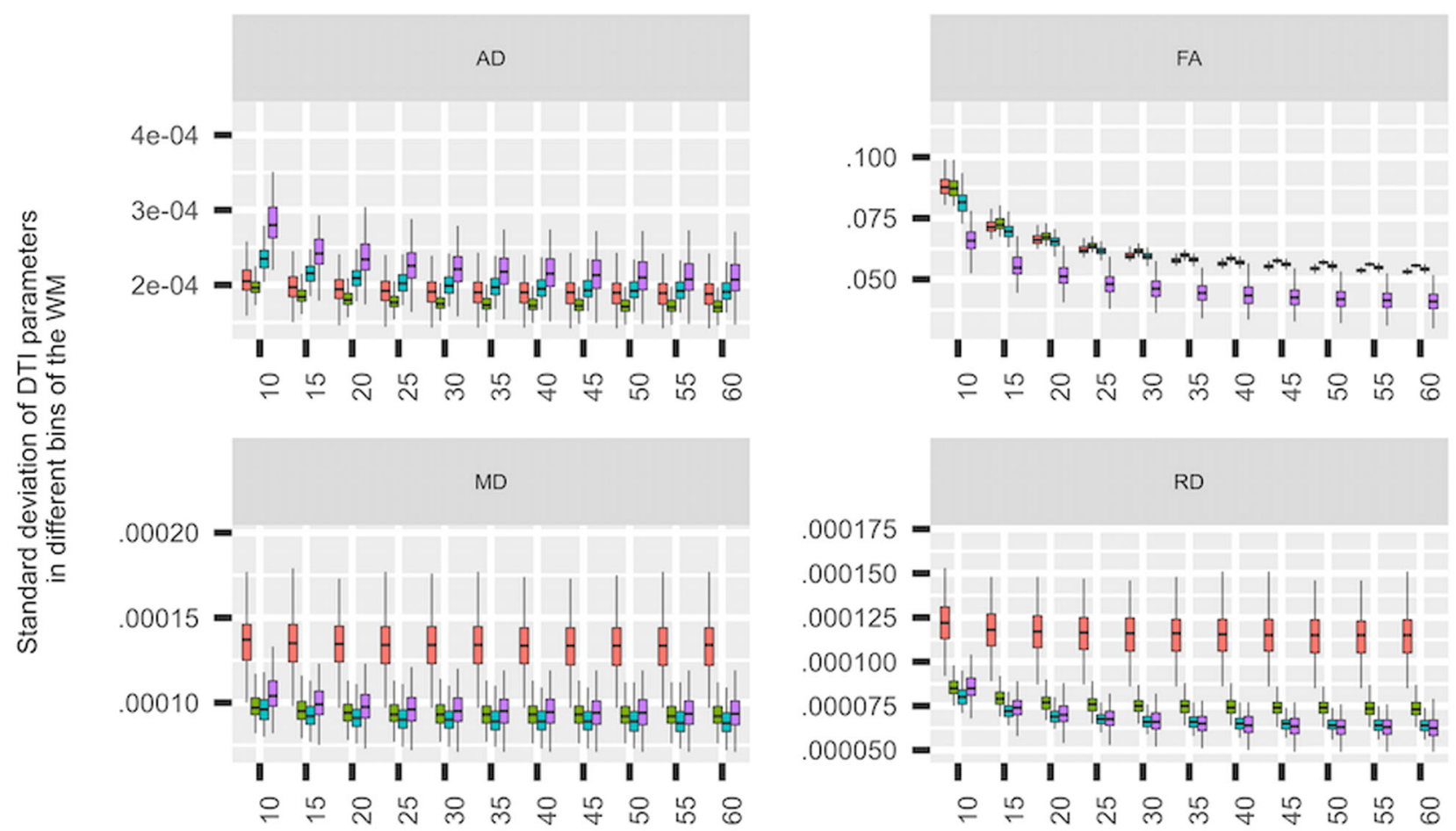

NDED

FA:0.2-0.4

FA:0.4-0.6

FA:0.6-0.8

FA:0.8-1

Fig 3. Standard deviation of DTI parameters. The standard deviation of the DTI parameters in separate bins of the white matter (WM) in healthy controls.

35, and 20 directions did not significantly differ from the 60 directions based on post hoc analysis (Tukey method) in AD, FA, MD, and RD, respectively (Fig 1). The asterisk in Figure 1 depicts NDED groups that differ significantly from the $60 \mathrm{di}$ rections group (Fig 1). Mean values of DTI parameters under different bins of the white matter were evaluated (Fig 2). Lower FA bins had a greater overestimation with decreasing directions but other parameters did not differ substantially.

The standard deviation of DTI parameters in the white matter mask increased slightly with decreasing directions, which was more visible for FA and AD parameters (Fig 3). For the different intensity bins, the FA value had the lowest standard deviation in the highest bin compared to other bins. In $\mathrm{RD}$ and MD parameters, the three upper bins had similar but lower standard deviation compared to the lowest bin. In contrary, the highest bin had the highest standard deviation compared to other bins in $\mathrm{AD}$ (Fig 3). As a function of NDED, the standard deviation of the whole white matter did not change significantly from the 60 directions at 55, 20, 35, and 20 directions in $\mathrm{AD}$, $\mathrm{FA}, \mathrm{MD}$, and $\mathrm{RD}$ respectively as revealed with post hoc analysis (Tukey method).

The TBSS analysis between $78 \mathrm{MS}$ patients and $126 \mathrm{HCs}$ revealed slightly different results as a function of NDED when threshold values were kept constant. The qualitative analysis revealed minimal differences in the skeleton size and the number of significant voxels (Fig 4, right side). The total size of the skeleton was reduced with increasing number of diffusion di- rections. Similarly, the number of significantly different voxels was reduced. However, when the FA threshold was changed to keep the skeleton size constant, the number of significant voxels was similar in FA and $\mathrm{RD}$ but there was a drop at 15 directions in MD and AD (Fig 4, left side). Regarding the group differences, the mean FA value within the significant voxels of the skeleton showed a decreasing trend with the elevation of the encoding directions. This was more pronounced in AD but absent in MD and RD (Figs 5-7). In addition, the MS group had smaller values in any number of diffusion directions for $\mathrm{FA}$ and higher values for $\mathrm{AD}, \mathrm{MD}$, and $\mathrm{RD}$ on a group level (Fig 5). There was a statistical significant interaction between the effect of disease group and NDED in the equal skeleton size condition for all parameters $(\mathrm{AD}: F(3,606)=10.2, P<.0001$; FA: $F(3,606)=8.4, P<.001$; MD: $F(3,606)=2.9, P<.05)$; and RD: $F(3,606)=4.2, P<.01)$. In the unequal skeleton size condition, interaction could be observed for AD, FA, MD, and $\mathrm{RD}$ as well $(\mathrm{AD}: F(3,606)=10.2, P<.0001$; FA: $F(3,606)$ $=8.8, P<.0001$; MD: $F(3,606)=2.7, P<.05)$; and RD: $F(3,606)=4.8, P<.01)$. This means that the mean differences of the groups change differently as a function of directions (Fig 6) in both skeleton sizes. The result of the TBSS analysis of the FA in unequal skeleton size condition is depicted in Figure 7.

\section{Discussion}

In the present study, we investigated the effect of the number of diffusion directions on the estimated diffusion parameters, with 


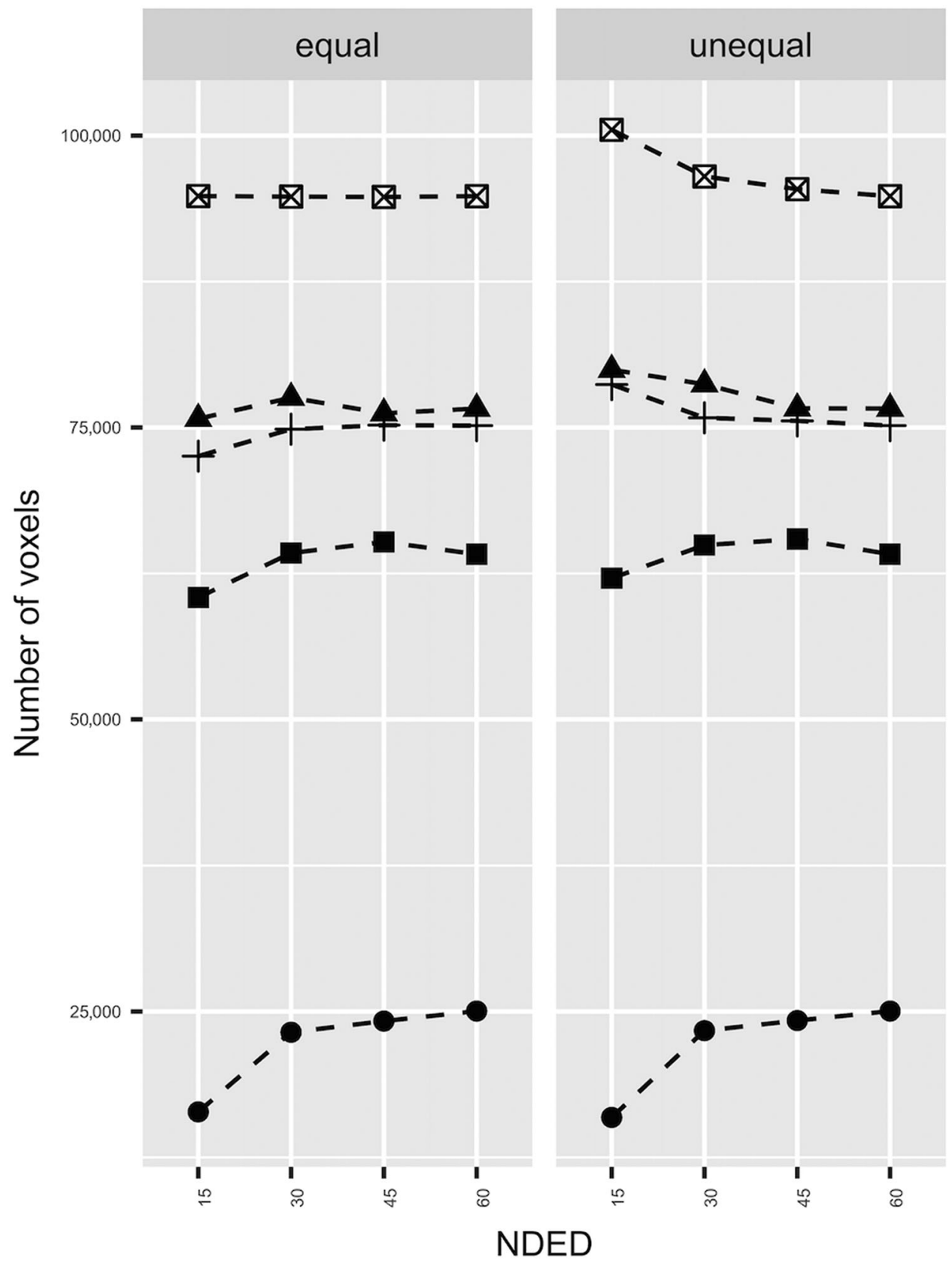

group - $A D \boldsymbol{\Delta} F A \quad M D+R D$ -

Fig 4. The results of TBSS analysis. The left side of the plot ("equal") shows the results of TBSS when skeleton size was held constant (see Methods). The right side of the plot ("unequal") shows the results when skeleton size was varying with NDED. The different groups represent the significant voxel count in that diffusion parameter. Correcting for skeleton size the number of significant voxel count did not change in FA and $R D$ as a function of NDED and the trend of $A D$ and MD parameter were the same.

a special emphasis on detecting differences in a patient population with the commonly used TBSS approach. We found an overestimation of FA and $\mathrm{AD}$ with .01 and $10^{-5}$, respectively, if the NDED was below 30 directions. Areas with low FA values seemed to be more prone to the overestimation. For RD, underestimation was found and MD was not affected by the NDED. When considering the TBSS analysis, the overestimation of FA leads to an enlarged skeleton and within that skeleton more significantly different voxels can be found at low NDED.

At least six noncollinear encoding directions are necessary to estimate the diffusion tensor. ${ }^{23}$ Moreover, previous studies with real-life data ${ }^{5,8,9,24}$ investigated the effects on NDED on accurate and precise FA estimation in ROI analysis. The decrease of the encoding directions leads to an overestimation of the FA and 

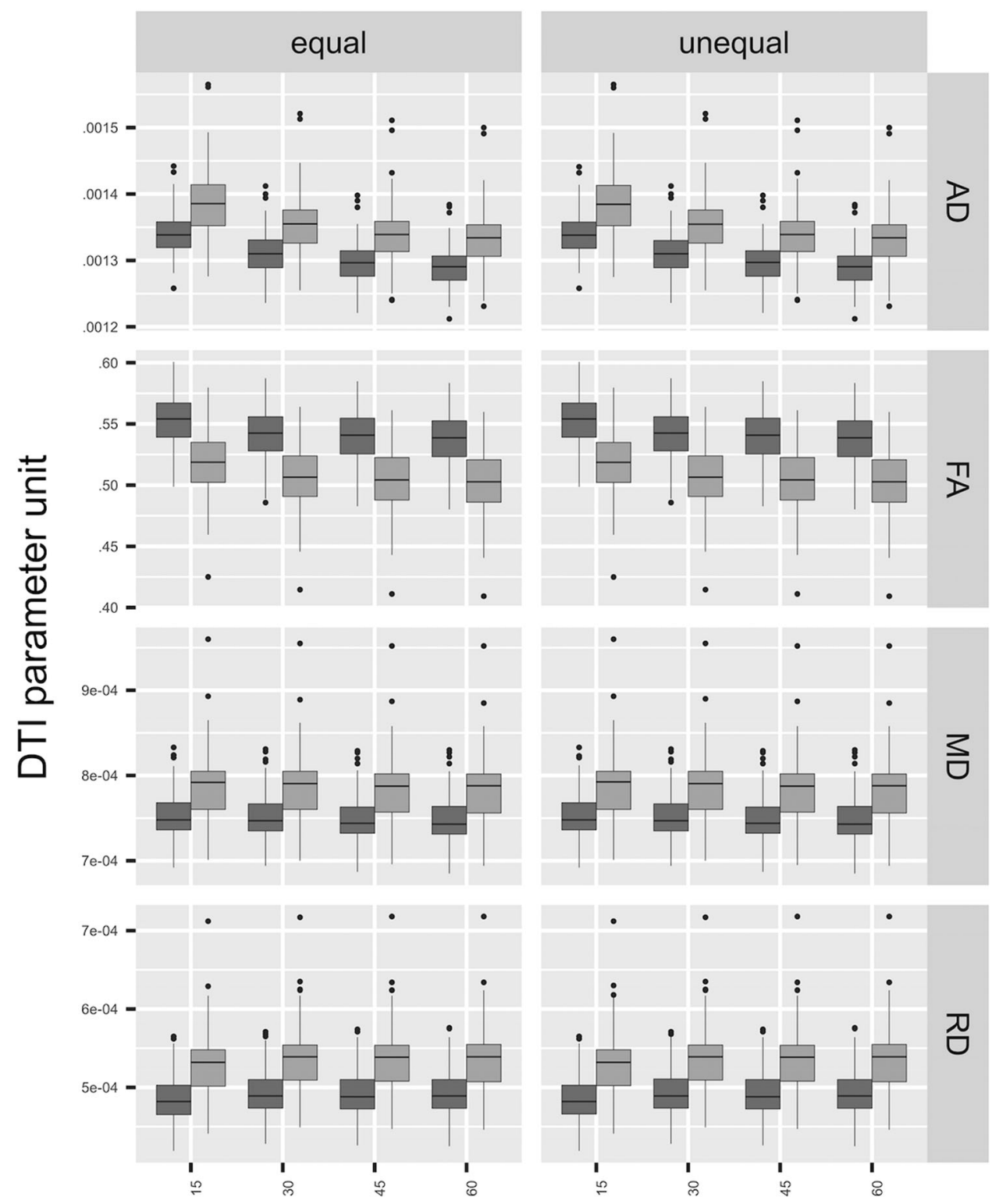

NDED

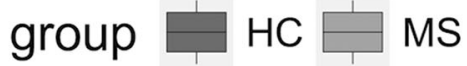

Fig 5. The mean FA under significant voxels. The left side of the plot ("equal") shows the distribution of the mean of DTI parameters under significant voxels when skeleton size was held constant (see Methods). The right side of the plot ("unequal") shows the same results when skeleton size was varying with NDED.

$\mathrm{AD}$ and an underestimation of RD but does not affect MD. The overestimation seems to be more severe if the uniform spherical distribution of directions has not been kept. ${ }^{24}$ As it was emphasized, the relationship of the fiber orientation and the encoding direction has a major effect on the tensor, especially in low NDED. Simulation studies found that the relationship of diffusion scheme and the underlying fiber orientation has a profound effect on the accuracy and precision of the estimated anisotropy. ${ }^{7}$ However, the investigation of the performance of different schemes on real-life data is more complicated because of the spatial and tissue-dependent SNR and other imaging- related artifacts such as chemical shift, distortion, and eddy currents.

The optimal NDED has been investigated in previous studies. ${ }^{5,8,9,24}$ In a ROI-based approach, no difference was found in FA and MD between 6 and 30 directions when scanning time was held constant at $1.5 \mathrm{~T} .{ }^{9}$ Moreover, they found an overestimation of the main eigenvalue (AD) in the six directions scheme in most of the ROIs. Another ROI approach found minimal but significant FA and MD changes between the 6 and 30 directions scheme at $1.5 \mathrm{~T} .{ }^{8}$ However, they concluded that the test-retest variability is higher than this difference. In 

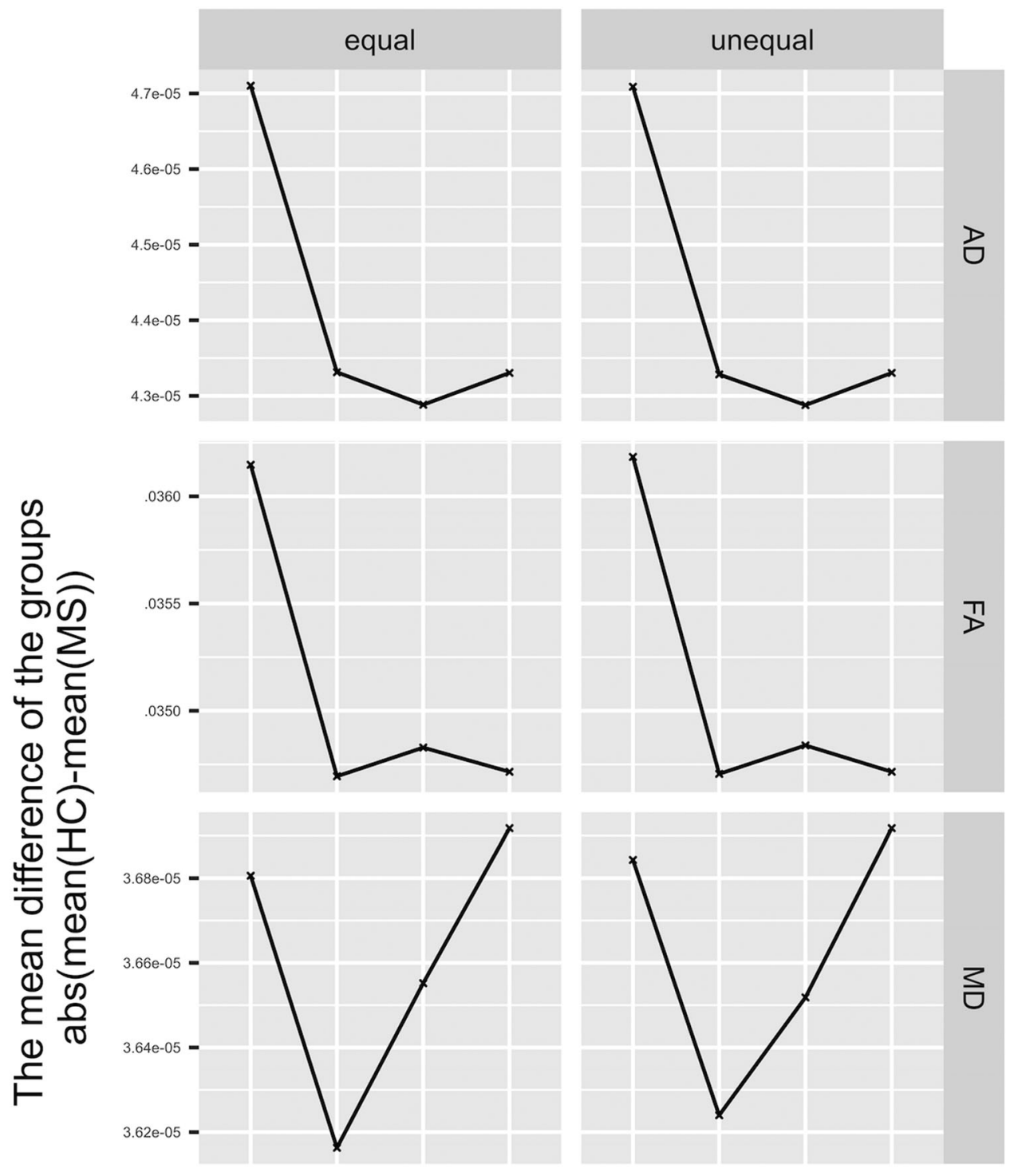

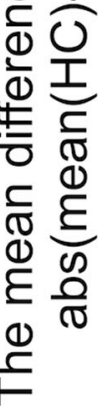
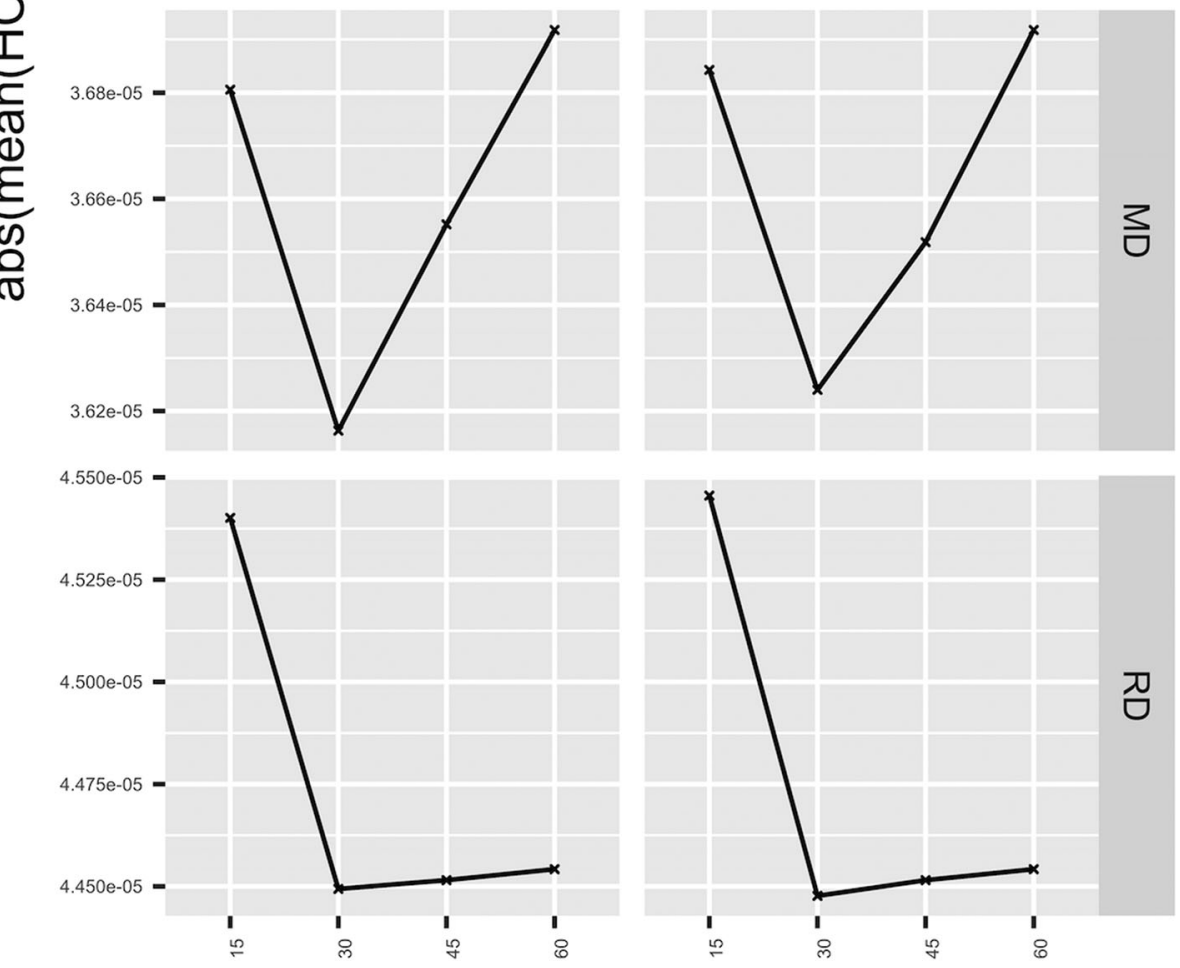

NDED

Fig 6. The mean difference of the groups. Left side represents the equal skeleton size and the right side represents the unequal skeleton size (see Methods). The parameters have various trends regarding the group difference. The skeleton size has no effect on the trends of the investigated parameters.

addition, an upward bias of FA could be detected in the gray matter as SNR decreased but no change was found in white matter ROIs. ${ }^{25}$ Therefore, the total number of scans was held constant for these studies, which resulted in a comparable SNR for the different schemes. Moreover, an increment in NDED was reported to lead to an elevation in SNR and to reach its plateau at 53 directions for FA and 51 directions for MD in the white matter. ${ }^{5}$ Random rejection of directions leads to an 


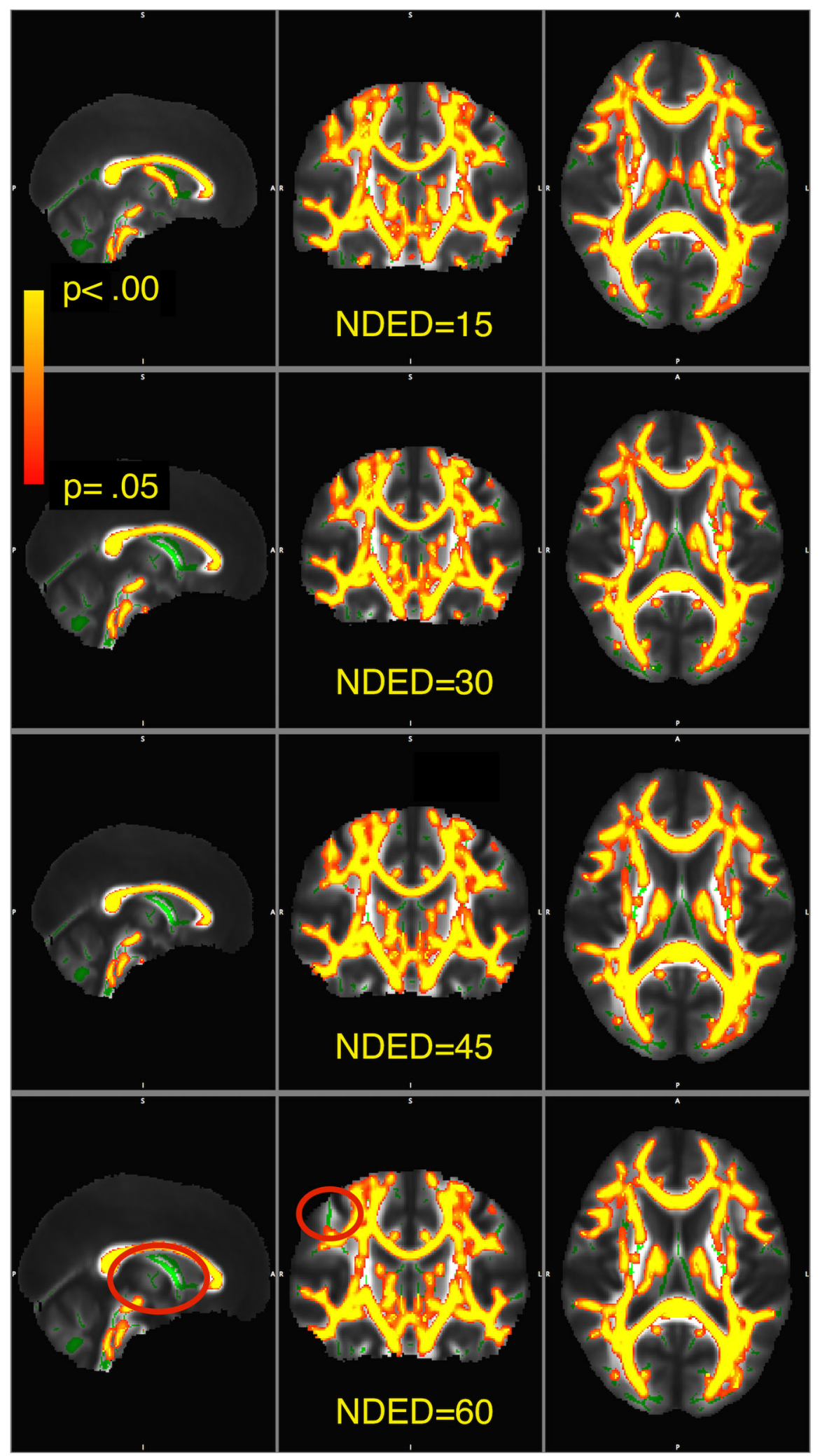

Fig 7. Visualization of significant voxels within the skeleton. The skeleton is showed in green. The statistical images are overlaid in yellow-tored and thickened with the tbss_fill function for visualization as recommended in FSL. The yellow-to-red colors represent $P$-values thresholded at $P<.05$ corrected. Red ovals show areas where significant voxels differ as a function of NDED. 
overestimation of FA and $\mathrm{AD}$ and an underestimation of $\mathrm{RD}$ but does not affect $\mathrm{MD}$; these effects are more apparent in areas with low FA. ${ }^{24}$ The uniform rejection has the same effects on low FA areas, but the overestimation is much lower. Lower SNR could be in the background of the overestimation with lower NDED. Moreover, FA and AD seem to be affected more profoundly by NDED in general than "isotropic indices" (MD and $\mathrm{RD}$ ), which averages information from more directions. The previously investigated ROIs mostly contain main tracts in which axons run in one direction. Nevertheless, the relative orientation of tracts and diffusion directions has an impact on the parameter estimation at low number of directions. ${ }^{7,8}$ However, the analyses of DTI data mostly use the full brain and TBSS is one popular method to do this. ${ }^{13}$ Therefore, to understand the effects of NDED on TBSS, analysis is crucial.

Diffusion parameters have a high spatial variability (eg, FA in the middle of the fiber bundle is much higher than at the periphery or, eg, over the anteroposterior aspect of the corpus callosum, ${ }^{26}$ FA changes together with the underlying histological features). This high spatial variability warrants voxel-wise statistics. However, registration in the white matter is not trivial. One possible solution is applied in the TBSS approach, namely, only the most structured parts of the white matter are investigated in the white matter skeleton. The skeleton is defined by the relatively high FA values (usually higher than .2-.3), which by itself reduces the bias, because areas with low FA values are more prone to the overestimation of FA.

MS is an inflammatory and neurodegenerative disease with various clinical symptoms in which white and gray matter are affected diffusely. ${ }^{15,27,28}$ The disease causes local lesions in the brain and alterations in the normal appearing white matter. ${ }^{29,30}$ Many studies found wide-spread changes in DTI parameters in MS. ${ }^{31-36}$ Here, we found extensive alteration of DTI parameters in the white matter in MS patients compared to HCs. The reason of this broad difference could be the high number of participants in the study, which may lead to the detection of smaller differences. Furthermore, the higher number of MS patients may also contribute because their inclusion leads to an increased percent of focal demyelinating lesions in the skeleton, which have reduced FA and increased MD. ${ }^{36}$ However, only a small proportion of the skeleton contains lesions $(7.5 \%$ of the total volume of the skeleton in a subpopulation of 40 MS patients from the current sample of whom manually segmented lesion mask were available). The mean difference in the significant voxels of FA and AD decreased with the NDED, that is, the two groups mean values depended on NDED in different way. In the lowest NDED, the group difference was the highest. One possible explanation could be the underlying pathology in MS. The white matter FA value is decreased throughout the brain, which is in turn, more prone to overestimation. Moreover, voxels included in the skeleton in the TBSS approach are more ambiguous. However, the significant change of group differences between the directions is still very low. Regarding the two skeleton sizes, the group differences of the parameters followed a similar trend. Moreover, the number of significant voxels and the skeleton size was different between different schemes and decreased with the NDED as well. The overestimation of skeleton size could be the consequence of the overestimation of FA within the small FA areas. In addition, the elevated number of significant voxels of FA simply resulted from the higher number of voxels. This effect could be reversed because correcting for skeleton size, the number of significant voxels of FA was equal as well. Conversely, the number of significant voxels of $\mathrm{MD}$ and $\mathrm{AD}$ was smaller at 15 directions compared to higher directions.

As we worked with fixed encoding directions and their subsets, the distribution of directions could differ from the optimal distribution. According to Zhan et al, this difference was in the range of 5-6\% in terms of distribution energy in 10 and 15 directions ${ }^{5}$ and as Zhan et al acknowledged that this "suboptimal sampling may play a minor role in the SNR gains." We repeated their calculation and the ratio of distribution energy in our study was less than $1 \%$, which is a negligible difference (Fig. S1). The difference between the two studies stems from the different number of encoding direction used in the original dataset, that is, 94 compared to 60 in our case.

One of the limitations of our study is that the results are only applicable to differences between MS and healthy patients; other diseases might show different pattern of TBSS' change as the function of NDED. Other parameters that have an effect on signal-to-noise should also be considered such as voxel size and field strength.

In conclusion, the bias caused by directions is more pronounced for areas with small FA values and seems to be constant above 30 directions. Moreover, NDED has a slight effect on TBSS, which makes it reasonable to cautiously compare results from different TBSS studies with different NDED. Regarding all the derived DTI parameters, 30 directions might be enough to compare healthy and MS patients with TBSS analysis. Importantly, our results indicate that higher FA threshold of the skeleton should be used with lower NDED to avoid false positive result.

\section{References}

1. Guo AC, MacFall JR, Provenzale JM. Multiple sclerosis: Diffusion tensor MR imaging for evaluation of normal-appearing white matter. Radiology 2002;222:729-36.

2. Szabó N, Kincses ZT, Párdutz Á, et al. White matter microstructural alterations in migraine: a diffusion-weighted MRI study. Pain 2012; 153:651-6.

3. Soares JM, Marques P, Alves V, et al. A hitchhiker's guide to diffusion tensor imaging. Front Neurosci 2013;7:31.

4. Jones DK, Horsfield MA, Simmons A. Optimal strategies for measuring diffusion in anisotropic systems by magnetic resonance imaging. Magn Reson Med 1999;42:515-25.

5. Zhan L, Leow AD, Jahanshad N, et al. How does angular resolution affect diffusion imaging measures? Neuroimage 2010;49:1357-71.

6. Hasan KM, Parker DL, Alexander AL. Comparison of gradient encoding schemes for diffusion-tensor MRI.J Magn Reson Imaging 2001;13:769-80.

7. Jones DK. The effect of gradient sampling schemes on measures derived from diffusion tensor MRI: a monte carlo study. Magn Reson Med 2004;51:807-15.

8. Landman BA, Farrell JAD, Jones CK, et al. Effects of diffusion weighting schemes on the reproducibility of DTI-derived fractional anisotropy, mean diffusivity, and principal eigenvector measurements at 1.5T. Neuroimage 2007;36:1123-38.

9. Ni H, Kavcic V, Zhu T, et al. Effects of number of diffusion gradient directions on derived diffusion tensor imaging indices in human brain. Am J Neuroradiol 2006;27:1776-81.

10. Papadakis NG, Murrills CD, Hall LD, et al. Minimal gradient encoding for robust estimation of diffusion anisotropy. Magn Reson Imaging 2000;18:671-9. 
11. Batchelor PG, Atkinson D, Hill DLG, et al. Anisotropic noise propagation in diffusion tensor MRI sampling schemes. Magn Reson Med 2003;49:1143-51.

12. Skare S, Hedehus M, Moseley ME, et al. Condition number as a measure of noise performance of diffusion tensor data acquisition schemes with MRI. J Magn Reson 2000;147:340-52.

13. Smith SM, Jenkinson M, Johansen-Berg H, et al. Tract-based spatial statistics: voxelwise analysis of multi-subject diffusion data. Neuroimage 2006;31:1487-505.

14. Tóth E, Faragó P, Király A, et al. The contribution of various MRI parameters to clinical and cognitive disability in multiple sclerosis. Front Neurol 2019;9:1172.

15. Tóth E, Szabó N, Csete G, et al. Gray matter atrophy is primarily related to demyelination of lesions in multiple sclerosis: a diffusion tensor imaging MRI study. Front Neuroanat 2017;11:23.

16. Jenkinson M, Bannister P, Brady M, et al. Improved optimization for the robust and accurate linear registration and motion correction of brain images. Neuroimage 2002;17:825-41.

17. Smith SM, Jenkinson M, Woolrich MW, et al. Advances in functional and structural MR image analysis and implementation as FSL. Neuroimage 2004(Suppl. 1):208-19.

18. Zhang Y, Brady M, Smith S. Segmentation of brain MR images through a hidden Markov random field model and the expectationmaximization algorithm. IEEE Trans Med Imaging 2001;20:45-57.

19. R Core Team. R: A Language and Environment for Statistical Computing. Vienna, Austria: R Foundation for Statistical Computing; 2018.

20. Bates D, Machler M, Bolker B, et al. Fitting linear mixed-effects models using lme4.J Stat Softw Artic 2015;67:1-48.

21. Searle SR, Speed FM, Milliken GA. Population marginal means in the linear model: an alternative to least squares means. Am Stat 1980;34:216-21.

22. Fox J, Weisberg S. An R Companion to Applied Regression. 2nd ed. Thousand Oaks CA: Sage; 2014.

23. Basser PJ, Mattiello J, Lebihan D. Estimation of the effective self-diffusion tensor from the NMR spin echo. J Magn Reson B 1994;103:247-54.

24. Chen Y, Tymofiyeva O, Hess CP, et al. Effects of rejecting diffusion directions on tensor-derived parameters. Neuroimage 2015;109:160-70.

25. Farrell JAD, Landman BA, Jones CK, et al. Effects of signal-tonoise ratio on the accuracy and reproducibility of diffusion tensor imaging-derived fractional anisotropy, mean diffusivity, and principal eigenvector measurements at 1.5T. J Magn Reson Imaging 2007;26:756-67.

26. Pfefferbaum A, Sullivan EV. Increased brain white matter diffusivity in normal adult aging: relationship to anisotropy and partial voluming. Magn Reson Med 2003;49:953-61.

27. Calabrese M, Filippi M, Gallo P. Cortical lesions in multiple sclerosis. Nat Rev Neurol 2010;6:438-44.

28. Popescu V, Agosta F, Hulst HE, et al. Brain atrophy and lesion load predict long term disability in multiple sclerosis. J Neurol Neurosurg Psychiatry 2013;84:1082-91.

29. Laganà M, Rovaris M, Ceccarelli A, et al. DTI parameter optimisation for acquisition at 1.5T: SNR analysis and clinical application. Comput Intell Neurosci 2010;2010:23.

30. Werring DJ, Clark CA, Barker GJ, et al. Diffusion tensor imaging of lesions and normal-appearing white matter in multiple sclerosis. Neurology 1999;52:1626-32.

31. Huang J, Liu Y, Zhao T, et al. White matter microstructural alterations in clinically isolated syndrome and multiple sclerosis. J Clin Neurosci 2018;53:27-33.

32. Preziosa P, Rocca MA, Mesaros S, et al. Intrinsic damage to the major white matter tracts in patients with different clinical phenotypes of multiple sclerosis: a voxelwise diffusion-tensor MR study. Radiology 2011;260:541-50.

33. Roosendaal SD, Geurts JJG, Vrenken H, et al. Regional DTI differences in multiple sclerosis patients. Neuroimage 2009;44:1397-403.

34. Bodini B, Khaleeli Z, Cercignani M, et al. Exploring the relationship between white matter and gray matter damage in early primary progressive multiple sclerosis: an in vivo study with TBSS and VBM. Hum Brain Mapp 2009;30:2852-61.

35. Schoonheim MM, Vigeveno RM, Rueda Lopes FC, et al. Sexspecific extent and severity of white matter damage in multiple sclerosis: implications for cognitive decline. Hum Brain Mapp 2014;35:2348-58.

36. Rovaris M, Gass A, Bammer R, et al. Diffusion MRI in multiple sclerosis. Neurology 2005;65:1526-32.

\section{Supporting Information}

Additional supporting information may be found online in the Supporting Information section at the end of the article.

Supplementary Material 